\title{
Coral reef soundscapes: spatiotemporal variability and links to species assemblages
}

by

Maxwell Bernard Kaplan

B.Sc., University of St Andrews (2012)

Submitted in partial fulfillment of the requirements for the degree of

Doctor of Philosophy

at the

MASSACHUSETTS INSTITUTE OF TECHNOLOGY

and the

WOODS HOLE OCEANOGRAPHIC INSTITUTION

February 2017

(C)2017 Maxwell Bernard Kaplan.

All rights reserved.

The author hereby grants to MIT and WHOI permission to reproduce and to distribute publicly paper and electronic copies of this thesis document in whole or in part in any medium now known or hereafter created.

Author

Joint Program in Oceanography/Applied Ocean Science \& Engineering Massachusetts Institute of Technology

Woods Hole Oceanographic Institution January 4, 2017

Certified by

T. Aran Mooney Associate Scientist Woods Hole Oceanographic Institution Thesis Supervisor

Accepted by

Ann Tarrant

Chair, Joint Committee for Biological Oceanography Massachusetts Institute of Technology Woods Hole Oceanographic Institution 


\title{
Coral reef soundscapes: spatiotemporal variability and links to species
}

assemblages

by

\author{
Maxwell Bernard Kaplan
}

\begin{abstract}
Submitted to the MIT-WHOI Joint Program in Oceanography and Applied Ocean Science and Engineering on January 4, 2017, in partial fulfillment of the requirements for the degree of Doctor of Philosophy in Biological Oceanography
\end{abstract}

\begin{abstract}
Coral reefs are biodiverse ecosystems that are at risk of degradation as a result of environmental changes. Reefs are constantly in a state of flux: the resident species assemblages vary considerably in space and time. However, the drivers of this variability are poorly understood. Tracking these changes and studying how coral reefs respond to natural and anthropogenic disturbance can be challenging and costly, particularly for reefs that are located in remote areas. Because many reef animals produce and use sound, recording the ambient soundscape of a reef might be one way to efficiently study these habitats from afar. In this thesis, I develop and apply a suite of acoustics-based tools to characterize the biological and anthropogenic acoustic activity that largely comprises marine soundscapes. First, I investigate links between reef fauna and reef-specific acoustic signatures on coral reefs located in the U.S. Virgin Islands. Second, I compare those findings to a more expansive study that I conducted in Maui, Hawaii, in which the drivers of bioacoustic differences among reefs are explored. Third, I investigate the distances over which sounds of biological origin may travel away from the reef and consider the range within which these acoustic cues might be usable by pelagic larvae in search of a suitable adult habitat. Fourth, I assess the extent to which the presence of vessel noise in shallow-water habitats changes the ambient soundscape. Finally, I present the results of a modeling exercise that questions how ocean noise levels might change over the next two decades as a result of major projected increases in the number and size of and distance traveled by commercial ships. The acoustics-based tools presented here help provide insight into ecosystem function and the extent of human activity in a given habitat. Additionally, these tools can be used to inform an effective regulatory regime to improve coral reef ecosystem management.
\end{abstract}

Thesis Supervisor: T. Aran Mooney

Title: Associate Scientist

Woods Hole Oceanographic Institution 


\section{Acknowledgments}

My doctoral studies were supported by the BP-MIT Energy Fellowship, the MIT Martin Family Fellowship, NSF grant OCE-1536728, NSF Ocean Acidification grant 1220034, Office of Naval Research award number N000141110612 and the WHOI Academic Programs Office. My research was funded by the Mitsubishi Corporation Foundation for the Americas, the PADI Foundation, the WHOI Access To The Sea Fund, the WHOI Coastal Ocean Institute, the WHOI Independent Study awards, the WHOI Ocean Life Institute, and the WHOI Ocean Ventures Fund.

My time at WHOI and MIT has been personally and intellectually enriching, and for that I have a number of people to thank.

My advisor, Aran Mooney, first gave me the opportunity to come to WHOI as a Summer Fellow and then to come back as a Joint Program student. Thank you for mentoring me and for supporting my research.

My committee - David Mann, Susan Solomon, Andy Solow, and Sofie Van Parijs provided crucial advice and support. Their expertise and input have greatly improved the work presented here. Michael Moore deftly chaired my thesis proposal defense and then my thesis defense.

This work could not have been completed without help from a number of colleagues. Frants Jensen was singularly generous with his time. Jim Partan's innovative (and largely pro bono) engineering work made much of this data collection possible. Marc Lammers was an immensely supportive collaborator. Thank you. There are many others to whom I am grateful, including Keenan Ball, Alessandro Bocconcelli, Daniel Bogorff, Tom DeCarlo, LiLing Hamady, Ed O’Brien, Laela Sayigh, Samantha Zacarias, Eden Zang, and Walter Zimmer.

Jim Yoder, Meg Tivey, Lea Fraser, Christine Charette, Julia Westwater, and Valerie Caron in the WHOI Academic Programs Office make being a Joint Program student easy and gave me the latitude to take advantage of the opportunities afforded by the unique nature of this program.

Thanks are also due to the WHOI Biology Department, in particular to Drew Brown for his patient and regular help, and to Becky Gast for her work as education coordinator.

My friends in Boston and Woods Hole have made being a Joint Program student fun 
and I am grateful for that.

Finally, my parents' advice and support over the last several years motivated me throughout my doctoral studies. Thank you. 


\section{Contents}

1 Introduction $\quad 15$

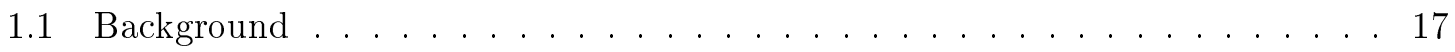

1.1.1 Coral reef ecology . . . . . . . . . . . . . . . . . . 17

1.1.2 Biological sound production . . . . . . . . . . . . . . 17

1.1.3 Reef soundscapes and biodiversity . . . . . . . . . . . 18

1.1.4 The propagation of reef soundscapes . . . . . . . . . . . . . 19

1.1.5 Anthropogenic noise . . . . . . . . . . . . . . . 20

1.1.6 Practical considerations . . . . . . . . . . . . . . . . 21

1.2 Thesis Overview . . . . . . . . . . . . . . . . 22

2 Coral reef species assemblages are associated with ambient soundscapes 25

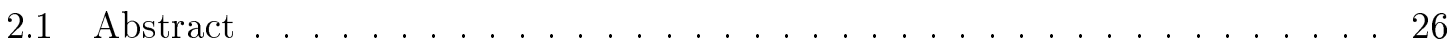

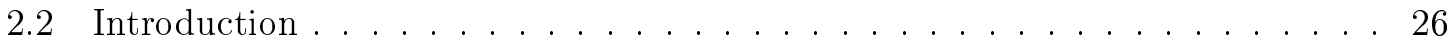

2.3 Methods . . . . . . . . . . . . . . . . . . . . 29

2.3.1 Visual surveys . . . . . . . . . . . . . . . . . . . . 29

2.3.2 Acoustic recordings . . . . . . . . . . . . . 30

2.3 .3 Acoustic analyses . . . . . . . . . . . . . . . . . . 31

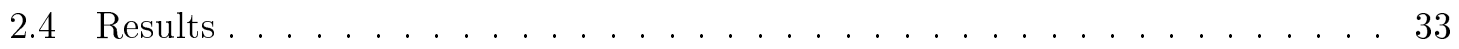

2.5 Discussion . . . . . . . . . . . . . . . . . . 40

2.6 Conclusion . . . . . . . . . . . . . . . . . . 46

2.7 Supplementary Material . . . . . . . . . . . . . . . . . . . 47

2.7.1 Supplementary figure . . . . . . . . . . . . . . . . . . . 47

2.7 .2 Non-stationarity analysis . . . . . . . . . . . . . . . 48 
3 Acoustic and biological trends on coral reefs off Maui, Hawaii 51

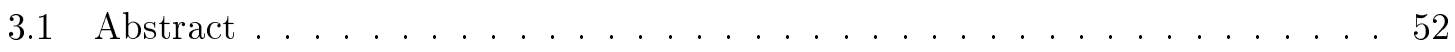

3.2 Introduction . . . . . . . . . . . . . . . . . . 52

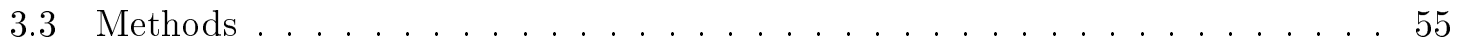

3.3 .1 Site selection . . . . . . . . . . . . . 55

3.3 .2 Visual surveys . . . . . . . . . . . . . . 55

3.3 .3 Acoustic data . . . . . . . . . . . . . . . . . 57

3.3.4 Environmental parameters . . . . . . . . . . . . . 59

3.3.5 Statistical analysis . . . . . . . . . . . . . . 59

3.4 Results . . . . . . . . . . . . . . . . . . . 60

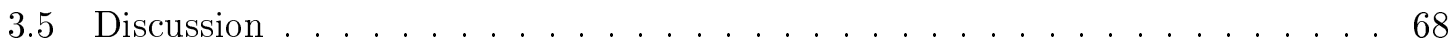

3.6 Supplementary Figures . . . . . . . . . . . . . . . . . . . 77

4 Coral reef soundscapes may not be detectable far from the reef $\quad 81$

4.1 Abstract . . . . . . . . . . . . . . . . . . 82

4.2 Introduction . . . . . . . . . . . . . . . . . . 82

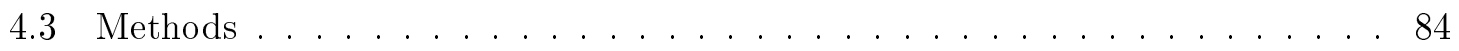

4.4 Results. . . . . . . . . . . . . . . . . . . . . 89

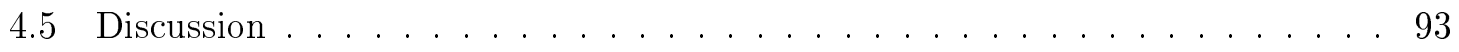

5 Ambient noise and temporal patterns of boat activity in the U.S. Virgin $\begin{array}{ll}\text { Islands National Park } & 99\end{array}$

5.1 Abstract . . . . . . . . . . . . . . . . . 100

5.2 Introduction . . . . . . . . . . . . . . . 100

5.3 Methods . . . . . . . . . . . . . . . . . . . . . . . 101

5.4 Results . . . . . . . . . . . . . . . . . . . . . . . 104

5.5 Discussion . . . . . . . . . . . . . . . . . 106

6 A coming boom in commercial shipping? The potential for rapid growth $\begin{array}{ll}\text { of noise from commercial ships by } 2030 & 115\end{array}$

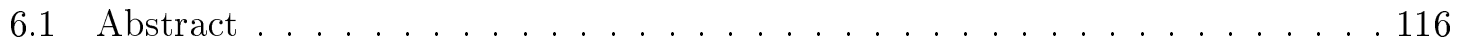

6.2 Introduction . . . . . . . . . . . . . . . . 116

6.3 Methods . . . . . . . . . . . . . . . . . . . 117 
6.4 Results \& Discussion . . . . . . . . . . . . . . . . . 118

6.5 Conclusions . . . . . . . . . . . . . . . . . . 121

7 Conclusion $\quad 123$

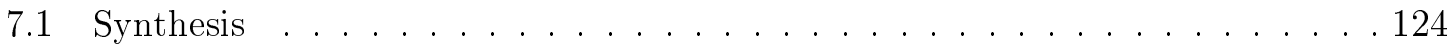

7.2 Nextsteps . . . . . . . . . . . . . . . . . . . . 127 
THIS PAGE INTENTIONALLY LEFT BLANK 


\section{List of Figures}

2.1 Map of study reefs in U.S. Virgin Islands . . . . . . . . . . . . . . . . . 34

2.2 Results of fish and benthic surveys . . . . . . . . . . . . . 35

2.3 Reef power spectra . . . . . . . . . . . . . . 36

2.4 Diel trend in biological sound levels in 2 frequency bands . . . . . . . . . . . 37

2.5 Correlation between sound production and reef biota . . . . . . . . 38

2.6 Octave band levels during the new and full moon $\ldots \ldots$. . . . . . . . . 39

2.7 Power spectral density heatmaps for a 24 -hour period at 3 reefs . . . . . . . 41

2.8 Long-term power spectral density estimates at 2 frequencies . . . . . . . . . 44

2.9 Acoustic entropy in 2 frequency bands . . . . . . . . . . . . . . . 45

2.S1 Relationship between high-frequency sound pressure level and wind speed . . 47

3.1 Map of study reefs in Maui . . . . . . . . . . . . . . . . 55

3.2 Visual survey results . . . . . . . . . . . . . . . . . . . 61

3.3 Relationship between soniferous fish and coral cover . . . . . . . . . . . 62

3.4 Seasonal trends in sound pressure level . . . . . . . . . . . . . . . . 63

3.5 Linear regression estimates for relationships between temperature and sound

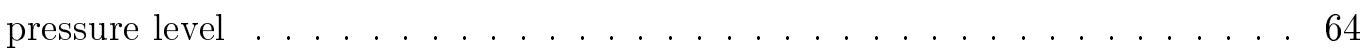

3.6 Lunar and diel periodicity in sound pressure level . . . . . . . . . . . . 65

3.7 Trends in diel sound pressure level in 2 frequency bands . . . . . . . . . . 66

3.8 Representative spectrograms for Olowalu and Honolua . . . . . . . . . . . . 67

3.9 Trends in diel median absolute deviations in 2 frequency bands . . . . . . . 68

3.10 Representative spectrograms for MM17 and Honolua . . . . . . . . . . . . . . 69

3.11 Periodograms showing relative strength of diel and lunar periodicity at Kahekili 70

3.12 CART regression tree for low-frequency sound parameters . . . . . . . . 73

3.13 CART regression tree for high-frequency sound parameters . . . . . . . . . . 74 
3.14 Predictor variable importance . . . . . . . . . . . . . . . . 75

3.S1 Correlations between wind speed and high-frequency sound pressure level _ . 77

3.S2 Correlations between temperature and low-frequency sound pressure level . . 78

3.S3 Correlations between temperature and high-frequency sound pressure level . . 79

4.1 Acoustic data collection schematic . . . . . . . . . . . . . . 85

4.2 Map of study site . . . . . . . . . . . . . . . . . . 87

4.3 Sound pressure level and power spectral density transect results . . . . . . . . 90

4.4 Particle velocity by distance . . . . . . . . . . . . . . . . . . . . . 91

4.5 Particle acceleration by frequency . . . . . . . . . . . . . . . 94

4.6 Sound pressure level calculated in short windows in 2 frequency bands . . . . 97

5.1 Map of acoustic recorder deployment locations in U.S. Virgin Islands . . . . . 103

5.2 Vessel noise presence summary _ . . . . . . . . . . . . . . . . . 105

5.3 Reef background noise levels . . . . . . . . . . . . . . . . . . 107

5.4 Sound pressure level in the presence and absence of vessels . . . . . . . . . . 108

5.5 Peak frequency in the presence and absence of vessels . . . . . . . . . . . 109

5.6 Spectrograms with vessels present and absent . . . . . . . . . . . . 110

5.7 Vessel noise spectrograms and power spectra . . . . . . . . . . . . . 113

5.8 Overlap between vessel noise spectra and fish audiograms . . . . . . . . . . 114

6.1 Commercial shipping fleet size and ocean ambient noise . . . . . . . . . . 119 


\section{List of Tables}

2.1 Summary of visual survey transects . . . . . . . . . . . . . 31

2.2 Correlations between the strength of the acoustic diel trend and the biota

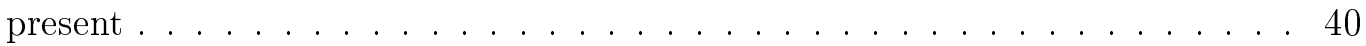

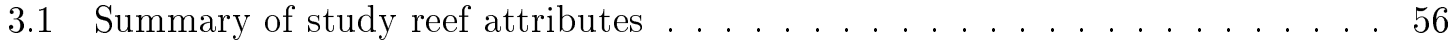

3.2 Acoustic data collection summary . . . . . . . . . . . . . . . 58

4.1 Data collection summary . . . . . . . . . . . . . . . 86

4.2 Sound pressure level and particle velocity results . . . . . . . . . . . . . . 92

4.3 Equations of decay curves fit to sound pressure level data . . . . . . . . . . 92

5.1 Boat noise occurrences and proportion of recording time with boat noise by

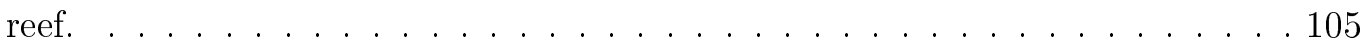

5.2 Sound pressure levels in 3 frequency bands at 3 reefs. . . . . . . . . . . . 106

5.3 Comparison of sound pressure level and peak frequency in the presence and absence of vessels . . . . . . . . . . . . . . . . . . . . 111

6.1 Projected fleet-wide source levels in $2030 \ldots \ldots$. . . . . . . . . . . 120

6.2 Projected sound exposure levels in $2030 \ldots \ldots$. . . . . . . . . . 120 
THIS PAGE INTENTIONALLY LEFT BLANK 


\section{Chapter 1}

\section{Introduction}


Patchiness in space and time is a defining feature of life in the marine environment. This is as true for ephemeral spring phytoplankton blooms as it is for patterns of sound production by marine animals. Variability in the dominant sound levels and frequencies in marine habitats can be appreciable; understanding the drivers of this variability may therefore provide fundamental insight into marine ecology.

Biological sound production at the community level (i.e., the soundscape generated by the local assemblage of soniferous species) has been shown to vary over time. Early work on this subject identified biological choruses, during which sound levels were approximately 30 decibels $(\mathrm{dB})$ above background noise, between sunset and midnight over several years of recordings of tropical waters north of Australia (Cato, 1978). Recordings of Hawaiian coral reefs contained peaks in fish sound production around sunrise and sunset (Au et al., 2012), underscoring the potentially important role of diel cycles in sound production. In addition to diel periodicity in sound level, lunar and seasonal variation in sound production has been demonstrated on coral and temperate reefs (Staaterman et al., 2014; Bohnenstiehl et al., 2016).

Time, then, is clearly important. However, sound levels in the marine environment also vary considerably in space. Radford et al. (2010) identified spectral and temporal differences between nearby sandy, macroalgal- and urchin-dominated habitats. Similarly, acoustic differences have been found between and among nearby coral reefs (Radford et al., 2014; Staaterman et al., 2014). An investigation using drifting acoustic recorders found increased sound levels in the vicinity of oyster reefs compared to soft-bottom habitat (Lillis et al., 2014).

Species-specific behavior contributes to these broad trends in biological sound production. Biotic sounds are produced by a wide range of taxa, including invertebrates and fishes (Tyack, 1998), in combination with a diverse array of biological processes, including spawning events (Lobel, 1992), courtship behaviors (Mann and Lobel, 1997), feeding (Versluis, 2000), and competition (Johnston and Vives, 2003).

Non-biological sound sources, such as noise from wind, waves, and anthropogenic activity (e.g., vessels) will also vary in space and time in terms of their contributions to the overall soundscape and levels relative to biotic sounds (Urick, 1984).

What drives variability in the oceans? While this question has been an underlying feature of much oceanographic research, it has only recently been asked of marine soundscapes. 
Given the relative ease with which sound in the ocean can now be recorded, identifying the biophysical drivers of spatiotemporal variability in biological sound production has become a research area of pressing ecological and practical importance. This thesis attempts to answer 4 main questions:

1. What are the dominant periods of biological sound production on coral reefs?

2. Do biological soundscapes reflect the species assemblages present on reefs?

3. How far might these soundscapes travel away from the reef?

4. What is the impact of vessel noise on ambient soundscapes?

\section{$1.1 \quad$ Background}

\subsubsection{Coral reef ecology}

Biologists have long attempted to understand the biophysical drivers of coral reef fish species assemblages. Historically, reef fish abundance was thought to be a function of recruitment variability and mortality (Doherty and Fowler, 1994). However, the preponderance of evidence suggests that hard corals provide key habitat for many reef fishes and invertebrates, and both coral cover and architectural complexity play major roles in influencing fish assemblages (McCormick, 1994; Friedlander et al., 2003; Jones et al., 2004; Graham et al., 2006; Wilson et al., 2006; Chong-Seng et al., 2012). For example, evidence from a study using artificial reefs suggested that fish species richness was correlated with coral species richness (Messmer et al., 2011). Because these strong relationships between fish species assemblages and physical habitat attributes such as live coral cover exist, and because many fishes produce sound, passive acoustic monitoring might represent a powerful tool for inverse prediction of species assemblages based on acoustic analyses of the diversity and occurrence of sounds produced by coral reef fish and other species. Such analyses may also allow for the detection of habitat changes on a fine scale and over extended periods of time.

\subsubsection{Biological sound production}

Sound production abilities vary among marine fauna (Tyack, 1998). On coral reefs, sounds largely originate from 2 major taxonomic groups - snapping shrimp and fishes. Snapping 
shrimp (e.g., Alpheus heterochaelis) produce sound for intraspecific communication, territory defense, and to stun or kill prey (Herberholz and Schmitz, 1999). The snapping sound results from the collapse of a cavitation bubble, which forms during the rapid closure of the claw (Versluis, 2000). The source level (SL) can be as high as $190 \mathrm{~dB}$ re $1 \mu \mathrm{Pa} @ 1 \mathrm{~m}(\mathrm{Au}$ and Banks, 1998), and snapping shrimp sounds are ubiquitous in the world's tropical and subtropical oceans, often dominating the soundscape in shallow coastal areas (Cato and Bell, 1992; Au and Banks, 1998).

Marine fishes that produce sound may do so in a number of ways, including through the contraction of sonic muscles attached to the swimbladder via the sonic ligaments (Parmentier et al., 2006). In general, most soniferous fishes show limited amplitude and frequency modulation abilities and have small vocal repertoires; however, these species may produce signals that are variable in the time domain for communication purposes (Amorim, 2006). Damselfish (Pomacentridae) are a particularly well-studied family, and many species produce a range of sound types at different times and under different circumstances (Mann and Lobel, 1997; Maruska et al., 2007; Myrberg, 1997; Parmentier et al., 2010). Hamlet (Hypoplectrus unicolor), which spawn in monogamous pairs during the crepuscular period, produce sound with every mating event (Lobel, 1992). Striped parrotfish (Scarus iserti) spawn in groups of 5-30 individuals, and sound production occurs during the spawning rush, in which the parrotfish swim upward rapidly en masse (Lobel, 1992). Sound production abilities are indeed common among fishes; for example, soniferous behaviors have been characterized for 45 species in 12 families of Hawaiian reef fish, representing just shy of half of the total number of species surveyed (Tricas and Boyle, 2014).

\subsubsection{Reef soundscapes and biodiversity}

Efforts devoted to recording and characterizing reef sounds and relating those sounds to reef species assemblages have been growing in recent years. An early study, based on short, daytime recordings from coral reefs, identified positive correlations between coral cover, fish density, and sound intensity (Kennedy et al., 2010); however, those acoustic records were too brief to account for the high temporal variability in biotic sound production that is found in many locations (Radford et al., 2008; Au et al., 2012; Staaterman et al., 2013). More recent work has identified appreciable variability within and among reefs over longer timescales (Staaterman et al., 2014; Nedelec et al., 2015). However, there is still a dearth of 
understanding of the biophysical drivers of sound production and the extent to which inverse prediction of reef species assemblages and ecological dynamics based on acoustic recordings might be possible.

\subsubsection{The propagation of reef soundscapes}

In addition to potentially facilitating the study of biodiversity, biological soundscapes may be used by larval fish to identify a suitable settlement location. The combination of sounds produced by marine organisms - the biological soundscape - could potentially be detectable far from the reef, and there is evidence to suggest that some larval invertebrates and fishes use this sound to locate and orient to settlement habitats (e.g., Simpson et al., 2005).

While planktonic fish larvae were once considered truly planktonic, with their trajectories determined only by the movement of the parcel of water in which they were entrained, the results of recent work demonstrate that larval fishes can regulate their depth in the water column (Paris and Cowen, 2004; Irisson et al., 2010; Huebert et al., 2011) and possess directional swimming abilities (Leis et al., 1996; Stobutzki and Bellwood, 1997; Leis and Carson-Ewart, 2003; Leis, 2006). Moreover, there is ample evidence that late-stage, nearsettlement larvae possess acute sensory and orientation capabilities that are integral to a larva's ability to locate suitable settlement habitat (Tolimieri et al., 2000; Atema et al., 2002; Kingsford et al., 2002; Simpson et al., 2004; Luczkovich et al., 2008; Leis and Lockett, 2005; Simpson et al., 2005; Montgomery et al., 2006; Gerlach et al., 2007; Mouritsen et al., 2013). Results from a range of experiments suggest that sound may be used by larvae (Simpson et al., 2004, 2005; Vermeij et al., 2010; Radford et al., 2011a); however, the distances over which acoustic cues might play a role are not known.

Sound fields are inherently composed of both a pressure and particle motion component, but the particle motion component has been typically paid scant attention, despite the fact that it is likely the primary acoustic stimulus for all sound-sensitive invertebrates and fishes (Nedelec et al., 2016). Propagation of reef sounds is likely to vary considerably based on source amplitude and spectral characteristics, bathymetry, bottom type, sea state, temperature, and salinity (Rogers and Cox, 1988). While some recent investigations of fish orientation behavior have directly measured particle motion in the lab (e.g., Zeddies et al., 2012; Coffin et al., 2014), until recently there have been no direct field measurements of coral reef acoustic particle motion. Such field measurements of both particle motion and sound 
pressure at a range of distances from a reef are required to assess the distances over which reef sound might act as a relevant cue for settlement-stage larval fishes and invertebrates.

\subsubsection{Anthropogenic noise}

In addition to biological soundscapes, other sound sources in the ocean might affect the behavior of marine fauna and impact our ability to understand and interpret sounds of biological origin. Noise resulting from anthropogenic activity in and adjacent to the oceans might directly affect a range of marine taxa (reviewed in Richardson, 1995; Hildebrand, 2004; Nowacek et al., 2007; Ladich, 2013; Kunc et al., 2016). Vessel noise playbacks have been found to disrupt feeding behavior in shore crabs (Carcinus maenas) (Wale et al., 2013), orientation behavior of cardinalfish (Apogon doryssa) (Holles et al., 2013), and antipredator behavior in European eels (Anguilla anguilla) (Simpson et al., 2015). North Atlantic right whales (Eubalaena glacialis) have been shown to call louder in the presence of elevated background noise (Parks et al., 2011). Vessel noise may affect embryonic development and increase mortality in sea hares (Stylocheilus striatus) (Nedelec et al., 2014). Temporary hearing threshold shifts after exposure to noise have been documented in aquatic fauna from goldfish (Carassius auratus) (Smith et al., 2004) to harbor seals (Phoca vitulina) (Kastak et al., 1999) and bottlenose dolphins (Tursiops truncatus) (Nachtigall et al., 2004). After September 11, 2001, when shipping traffic was temporarily halted, the corresponding 6 $\mathrm{dB}$ reduction in sound levels was correlated with reduced stress hormones in fecal samples retrieved from North Atlantic right whales (Rolland et al., 2012). Similarly, ship noise exposure has been linked to elevated stress hormone concentrations in the common carp (Cyprinus carpio) (Wysocki et al., 2006). Finally, exposure to high-intensity sounds such as pile driving has been associated with mortality in some fishes (reviewed in Popper \& Hastings 2009) and beaked whales (Cox et al., 2006).

Despite this wealth of experimental evidence for potentially adverse effects of noise on marine fauna, there have been only a very small number of studies that have attempted to quantify changes to ambient ocean noise levels. Recordings from the north Pacific Ocean indicated that low-frequency ambient noise levels increased by approximately $3 \mathrm{~dB}$ per decade from 1960-1990, potentially as a result of commercial shipping activity (McDonald et al., 2006). More recent estimates have suggested lower but positive rates of increase in that location (Andrew et al., 2011). Similarly, in the Atlantic Ocean, recordings taken near 
Bermuda have suggested low but positive rates of increase in ambient low-frequency ocean noise over time (S̊rović et al., 2016). However, there are few other studies from elsewhere in the world's oceans. Furthermore, these deep-water recordings likely do not reflect noise levels closer to shore where small vessel activity might cause high-amplitude but perhaps transient increases in ambient sound levels. Accordingly, alternative approaches to understanding both the impact of small-vessel activity on shallow-water soundscapes and the potential for ambient ocean noise to increase are needed.

\subsubsection{Practical considerations}

Tracking changes to marine communities poses many logistical and practical challenges, not least of which is the large geographic scale over which shallow-water marine ecosystems are found. The far-ranging distribution and isolation of many habitats makes conventional monitoring (e.g., visual surveys) logistically difficult, costly, and limited to daylight hours. Accordingly, finding effective new ways to identify and track marine biodiversity and health is of great importance. Acoustic recording technology may be one such method.

Acoustic data can provide detailed information on biological trends (Lammers et al., 2008). Recordings can be made in a range of conditions including at depth, at night, and in murky waters (Lammers et al., 2008). Locations can be monitored with acoustics for months or longer without human presence or disturbances (e.g., Parks et al., 2014). These recordings can be paired with additional physical measures to investigate broader ecosystem dynamics (e.g., Hofmann et al., 2013). Long-term recordings may be particularly useful to compare sound production levels before and after events such as storms (Locascio and Mann, 2005), habitat degradation (Ackleh et al., 2012), climate-related changes (Tittensor et al., 2010), or marine protection designation (Rayment et al., 2009). Thus, characterizing the bioacoustic signals produced in a given habitat may provide a low-cost method of tracking communities through space and time and could complement the broader shift towards the use of remote-sensing technology in reef research (Hedley et al., 2016).

Coral reefs are singularly amenable to bioacoustic research. They have been referred to as the "rainforests of the sea" because their productivity and biodiversity rivals that of their terrestrial counterpart. They host some of the highest diversity of life per unit area on Earth, and provide habitat for $25-30 \%$ of all marine animals, despite covering only $0.2 \%$ of the world's oceans (Reaka-Kudla, 1997; Knowlton et al., 2010; Plaisance et al., 2011). 
Reef-associated animals are a major source of protein for millions of people (WMO, 2010). In addition, reefs offer shoreline protection for communities and are a significant source of tourism revenue for developed and developing countries that may otherwise have no major industries (WMO, 2010). Globally, coral reef ecosystem services have been valued at USD $\$ 30$ billion per year (WMO, 2010).

Some of this value might be depleted as a result of stressors such as ocean warming (Jokiel and Coles, 1990), eutrophication (Szmant, 2002), overfishing (Harvell et al., 1999; Bruno et al., 2003, 2007; Hughes et al., 2003; Pandolfi et al., 2003), ocean acidification (Hoegh-Guldberg et al., 2007; Kroeker et al., 2013), and even anthropogenic noise (Simpson et al., 2016). Degradation of these ecologically and commercially important ecosystems threatens ecological balances and the human populations reliant on these marine resources, and underscores the need for further study.

\subsection{Thesis Overview}

This thesis quantifies the spatiotemporal variability in coral reef soundscapes. It relates that variability to the species assemblages present on reefs and identifies a series of putative biological drivers of reef-specific soundscapes. It assesses the distances over which reef sound might travel and the range over which such a cue may be useful for pelagic larvae in search of suitable benthic settlement habitat. It measures the occurrence of vessel noise on coral reefs and identifies the effects of that noise source on the overall soundscape. Finally, it examines how the growth of the commercial shipping fleet might result in elevated fleet-wide source levels.

In chapter 2, the relationship between reef species assemblages and ambient soundscapes is described based on data collected over a 4-month period from 3 reefs located in the U.S. Virgin Islands National Park. In chapter 3, that biological-acoustic relationship is further refined and elucidated through a long-term (approximately 16-month-long) study of 7 reefs located in Maui, Hawaii. In chapter 4, the distance over which reef sound pressure and particle motion might travel is quantified and the potential overlap with hearing thresholds of relevant marine species is estimated based on data collected from a single Hawaiian reef. In chapter 5 , the extent of vessel noise and its effects on the local soundscape are described for the same 3 reefs studied in chapter 2. Finally, in chapter 6 , projections going forward to 
2030 of the source and sound exposure levels of three segments of the global shipping fleet are presented. 
THIS PAGE INTENTIONALLY LEFT BLANK 


\section{Chapter 2}

\section{Coral reef species assemblages are}

\section{associated with ambient soundscapes}

This chapter was originally published as: Kaplan, M.B., Mooney, T.A., Partan, J., \& Solow, A.R. 2015. Coral reef species assemblages are associated with ambient soundscapes. Marine Ecology Progress Series 533:93-107. DOI: 10.3354/meps11382. This article is reprinted in this thesis under license from the copyright holder, Inter-Research. Under this license it is not permitted to further copy and distribute the entire article from this source separately from the thesis. This restriction ends 5 years after the date of original publication in Marine Ecology Progress Series. 


\subsection{Abstract}

Coral reefs provide a wide array of ecosystem services and harbor some of the highest levels of biodiversity on the planet, but many are in decline worldwide. Tracking changes is necessary for effective resource management. Biological sounds have been proposed as a means to quantify ecosystem health and biodiversity, but this requires a foundational understanding of natural bioacoustic variability and relationships to the taxa present. This investigation sought to characterize spatial and temporal variation in biological sound production within and among reefs that varied in their species assemblages. Multiple acoustic recorders were deployed for intensive $24 \mathrm{~h}$ periods and longer-term (ca. $4 \mathrm{mo}$ ) duty-cycled deployments on 3 reefs that varied in coral cover and fish density. Short-term results suggest that while there were statistically significant acoustic differences among recorders on a given reef, these differences were relatively small, indicating that a single sensor may be suitable for acoustic characterization of reefs. Analyses of sounds recorded over approximately 4 mo indicated that the strength of diel trends in a low-frequency fish band (100 to $1000 \mathrm{~Hz})$ was correlated with coral cover and fish density but the strength of high-frequency snapping shrimp (2 to $20 \mathrm{kHz}$ ) trends was not, suggesting that low-frequency recordings may be better indicators of the species assemblages present. Power spectra varied within reefs over the deployment periods, underscoring the need for long-duration recordings to characterize these trends. These findings suggest that, in spite of considerable spatial and temporal variability within reef soundscapes, diel trends in low-frequency sound production correlate with reef species assemblages.

\subsection{Introduction}

Coral reef ecosystems can support high biodiversity and provide key habitat for many taxa (Graham and Nash, 2013) as well as valuable ecosystem services (Graham et al., 2013). However, reefs are in decline worldwide as a result of multiple chronic and acute stressors (Bellwood et al., 2004; De'ath et al., 2012). Monitoring changes in reef ecosystems is a high priority, but traditional monitoring approaches can be intermittent and costly (Lammers et al., 2008). Biological sounds in the sea are produced by a diverse array of organisms and can play a vital role in the functioning of marine communities. This is because sound production is an important component of a number of biological processes, including spawn- 
ing events (Lobel, 1992), courtship behaviors (Mann and Lobel, 1997), feeding (Versluis, 2000), and competition (Johnston and Vives, 2003). Monitoring these sounds can reveal the occurrence of these behaviors, which are otherwise difficult to observe. Sounds can also be used to indicate the presence of certain taxa (e.g., Mann et al., 2009). Such information is valuable for species which are cryptic or require specific management. Unlike visual surveys, acoustic recordings can be collected without human presence, at night, and over long periods of time. However, to apply soundscape measurements as an ecological tool, it is necessary to understand the natural variability of those soundscape cues.

Biological sound production at the community level varies on multiple temporal and spatial scales. Fish sounds on Hawaiian coral reefs peak at crepuscular periods (Au et al., 2012), whereas fish calling activity on the Great Barrier Reef was found to be highest at midnight (McCauley and Cato, 2000). Radford et al. (2008) measured ambient noise at a temperate reef in New Zealand over an annual period, and found that the dominant biological sound sources were contained within 2 frequency bands: 700 to $2000 \mathrm{~Hz}$ (fish and urchins) and 2 to $15 \mathrm{kHz}$ (snapping shrimp), with diel and seasonal variation in amplitude. Sound production may be impacted by physical factors such as light (Staaterman et al., 2014); however, at present, the drivers of variability in sound production are mostly unknown.

Spatially, Radford et al. (2010) found spectral and temporal differences between nearby sandy, macroalgal- and urchin-dominated habitats. The frequency band associated with urchin feeding ( 800 to $2500 \mathrm{~Hz}$ ) had much greater sound intensity at the urchin-dominated habitat compared to other habitats, and the greatest differences among habitats were found at dusk (Radford et al., 2010). Similar among-site variability has been identified on coral reefs (Radford et al., 2014). Within-reef variability is also not well understood, but such an investigation is needed to determine how best to evaluate a soundscape. Given that settlement-stage fishes and invertebrates have been shown to use sound to locate and orient to settlement sites (Tolimieri et al., 2000, 2004; Simpson et al., 2004, 2005; Vermeij et al., 2010; Radford et al., 2011a), site-specific soundscapes may allow these animals to identify suitable habitats.

Despite goals to use marine soundscapes to evaluate community assemblages (McWilliam and Hawkins, 2013; Staaterman et al., 2014), acoustic recordings have not typically been paired with surveys of the species present in a given habitat, making it difficult to link species or community structure to sound production. One study that collected short (120 
s) recordings from coral reefs found a positive correlation between coral cover, fish density, and daytime sound intensity (Kennedy et al., 2010). A similar study using slightly longer recordings $(3 \mathrm{~min})$ collected from multiple sites and times of day and paired with visual surveys found relationships between acoustic parameters and the biota present (Nedelec et al., 2015). However, the brevity of the acoustic records used in these 2 studies likely overlooked the high temporal variability in biotic sound production that is typically found in many locations (Radford et al., 2008, 2014; Au et al., 2012; Staaterman et al., 2013). This variability suggests that longer-term recordings are better suited to determining whether acoustic differences among reefs are reflective of habitat differences and whether these differences persist over longer timescales.

As many fish species produce sound, the diversity of sound types may be indicative of fish assemblages (McWilliam and Hawkins, 2013) and perhaps overall community structure. Hard corals provide key habitat for many reef fish and invertebrates, and both coral cover and architectural complexity play major roles in influencing fish assemblages (e.g., McCormick, 1994; Friedlander et al., 2003; Jones et al., 2004). For example, in the Seychelles, fish species richness, functional group diversity, and abundance of corallivores decreased along a gradient of high to low coral cover and structural complexity, with very different fish assemblages at the habitat extremes (Chong-Seng et al., 2012). Evidence from a study using experimental reefs with variable numbers of coral species suggests that fish species richness, but not fish abundance or species evenness, is correlated with coral species richness (Messmer et al., 2011). Given these links between fish assemblages and coral cover/species richness, passive acoustic monitoring of the diversity and occurrence of sounds produced by coral reef fish may allow for overall habitat changes to be detected on a fine scale and over long periods of time.

To date, some effort has gone into the development of acoustic diversity indices, whereby acoustic records are used to make inferences about community biodiversity (Pijanowski et al., 2011). In theory, the use of an index allows for data reduction and improved ease of interpretation, which is desirable given that long-term acoustic monitoring produces vast quantities of data. Much of the work to date has focused on the terrestrial environment (Sueur et al., 2008; Depraetere et al., 2012; Gasc et al., 2013), but there have been some recent attempts to apply similar methods to marine bioacoustic recordings (McWilliam and Hawkins, 2013; Parks et al., 2014), with mixed success. 
To determine how species assemblages link to biological sound production, we deployed acoustic recorders on 3 reefs that varied in coral cover and fish density in the U.S. Virgin Islands National Park, a marine protected area which has been studied for several decades. Two general experiments were conducted: short-term $(24 \mathrm{~h})$, high-resolution measurements within each reef type and longer-term (ca. 4 mo) comparisons among the reefs. Acoustic recorder deployments were carried out in coordination with visual surveys of the benthos and fish assemblages in order to investigate the links between coral cover, fish abundance and diversity, and marine biological sound production. The utility of the acoustic entropy index (Sueur et al., 2008) and acoustic complexity index (Pieretti et al., 2011) was then evaluated as a means of interpreting these acoustic records.

\subsection{Methods}

To assess the relationship between species assemblages and coral reef bioacoustic soundscapes, this work focused on 3 reefs which varied in coral cover and fish abundance. Reefs were chosen based on long-term survey data (Edmunds, 2013) and a rapid, preliminary visual survey of 10 reefs in the area. Two of these - Tektite and Yawzi Point - have been studied for 25 yr (see Edmunds, 2013, for review). The third reef - Ram Head - was selected as a low coral cover comparison site and has been previously studied for larval settlement and current dynamics (Green and Edmunds, 2011). These reefs were chosen such that they varied as much as possible from each other in coral cover and fish density. All 3 reefs were located in the U.S. Virgin Islands National Park (Figure 2.1) and were similar in distance from shore and wave exposure. Tektite ranged from 9 to $18 \mathrm{~m}$ depth and consisted of a large sloping reef face, Yawzi ranged from ca. 5 to $10 \mathrm{~m}$ depth and was composed of a large mound that sloped down to sand, and Ram Head ranged from 8 to $13 \mathrm{~m}$ and was mostly flat, with patch reef sparsely located throughout the site. The 3 reefs are known to vary in coral settlement (Green and Edmunds, 2011).

\subsubsection{Visual surveys}

Benthic cover and fish abundance and diversity at each of the study sites were characterized through SCUBA-based visual surveys prior to instrument deployment and after recovery, following the standard Atlantic and Gulf Rapid Reef Assessment (AGRRA) methodology 
(available at www.agrra.org). At least 3 fish and benthic cover transects were carried out at each reef in both April and August 2013 (Table 2.1), and all surveys were carried out during daylight hours (09:00 to 17:00 h). Fish surveys consisted of $30 \mathrm{~m}$ transects (2 m wide) in which all fish along the transect were enumerated and classified to at least family level and size class. Benthic surveys consisted of $10 \mathrm{~m}$ long transects ( $1 \mathrm{~m}$ wide), with cover recorded at every $10 \mathrm{~cm}$ increment. Cover was later summarized into categories (coral, macroalgal, and other, which consisted primarily of crustose coralline algae, turf algae, other invertebrates, bare rock, dead coral, and sand). Surveys for fish and benthic cover were carried out adjacent to each other and to the acoustic recorder mooring (within a few meters). As there were no statistical differences between months, visual survey data were combined for analyses. Tests for significant differences among reefs in coral cover were conducted with a 1-way ANOVA. Fish survey data were analyzed by trophic level (herbivores, invertivores, consumers; data taken from FishBase available at www.fishbase.org) and summarized as fish density at each reef, based on the total area of transects. Visual survey data are presented as means ( \pm standard error). Differences in the distributions of fish at each trophic level among reefs were examined using a chi-square test. For both benthic and fish surveys, transects were randomized among reefs, and test statistics were recalculated 1000 times for increased robustness. The probability of getting the original statistical result by chance was determined by identifying the proportion of times that a randomized test statistic was greater than the original test statistic.

\subsubsection{Acoustic recordings}

Instrumentation. Acoustic measurements were made using DMON (Woods Hole Oceanographic Institution) and DSG (Loggerhead Instruments) recorders. For this investigation, the DMONs were configured with a low-noise preamplifier (20 dB gain), $13.2 \mathrm{~dB}$ user programmable gain, a 6-pole Sallen-Key anti-alias filter, a 16-bit analog-to-digital converter, and 32 GB of flash memory. We programmed the DMON to record on 2 hydrophone (Navy type II ceramics) channels: LF (16 kHz sample rate with an anti-aliasing filter at $7.5 \mathrm{kHz}$ and high pass filter at $8 \mathrm{~Hz})$ and $\mathrm{MF}(120 \mathrm{kHz}$ sample rate with an antialiasing filter at 50 $\mathrm{kHz}$ and high pass filter at $100 \mathrm{~Hz}$ ). The DSG records on a single channel $(80 \mathrm{kHz}$ sample rate) using a HTI-96 hydrophone (High-Tech) and contains a 16-bit computer board. There is a user-selectable gain setting; for these recordings, $20 \mathrm{~dB}$ was used, which resulted in a 
Table 2.1: Summary of visual survey transects undertaken in 2013 on 3 reefs in the U.S. Virgin Islands National Park.

\begin{tabular}{l|l|l|l|l|l|l}
\multicolumn{1}{c|}{ Site } & \multicolumn{2}{c|}{ Tektite } & \multicolumn{2}{c|}{ Yawzi } & \multicolumn{2}{c}{ Ram Head } \\
\hline Survey month & April & August & April & August & April & August \\
Number of fish transects & 8 & 3 & 9 & 4 & 5 & 4 \\
Number of benthic transects & 4 & 4 & 4 & 5 & 4 & 5
\end{tabular}

high-pass filter being implemented at $80 \mathrm{~Hz}$.

Deployments. Two sets of deployments were made. Short-term deployments used 4 continuously recording DMONs spaced approximately $20 \mathrm{~m}$ apart on each reef in both April and August for $24 \mathrm{~h}$ periods at a range of depths among the 3 reefs (ca. 5 to $14 \mathrm{~m}$ ). Because results were similar in April and August, only the latter results are presented here. Longterm records were collected using DMONs and DSGs recording on duty cycles at 2 locations per reef from April to August (ca. 8 to $12 \mathrm{~m}$ depth). Two concrete moorings were prepared for each site. Mooring 1 consisted of a DMON with customized duty-cycling software (2.5 min every $2 \mathrm{~h}$ ) and a DSG acoustic recorder (1 min every $20 \mathrm{~min}$ ) for redundancy in case of equipment failure. Mooring 2 consisted of a DMON only. Moorings were deployed between 17 and 19 April 2013 and retrieved between 2 and 3 August 2013, yielding approximately $103 \mathrm{~d}$ of potential data collection per site.

The only instrument to record at Tektite was the DSG. The redundancy of recorders proved essential as the DSGs deployed at Yawzi and Ram Head did not successfully record. As such, acoustic comparisons between sites involve multiple recording devices. The recording durations were as follows: Tektite, 19 April to 6 July 2013; Yawzi, 17 April to 1 August 2013; Ram Head, 19 April to 2 August 2013.

Temperature data loggers (HOBO pendant model UA-002-64, Onset Computer Corporation) were deployed with each acoustic recorder and sampled the ambient water temperature once every $10 \mathrm{~min}$.

\subsubsection{Acoustic analyses}

Analyses were carried out in Matlab 8.1 (MathWorks) using customized scripts written specifically for these data. Both short- and long-term DMON recordings were cut into 60 s segments. Boat noise and any other sporadic noise was identified visually using longterm spectral average (LTSA) plots implemented in Triton version 1.90 (Scripps Whale 
Acoustic Lab) and confirmed aurally. LTSAs were computed with $2 \mathrm{~s}$ averages and in 200 $\mathrm{Hz}$ bins. Sound files containing these anthropogenic and transient noises were excluded from all subsequent analyses but in some cases are included in visual representations of the soundscape for illustrative purposes. These steps resulted in the exclusion of $25 \%$ and $19 \%$ (short-term and long-term) of files from Tektite, 25\% and 7\% of files from Yawzi, and $8 \%$ and $9 \%$ of files from Ram Head. All remaining files were corrected for calibrated hydrophone sensitivity and resampled to $44 \mathrm{kHz}$. Spectral analysis used a Fast Fourier Transform (FFT) size of 880 points and a Hamming window with no overlap, yielding a spectral resolution of $50 \mathrm{~Hz}$ and a temporal resolution of 20 milliseconds (ms).

Likelihood ratio tests of the spectral density function of reef sound were used to test for temporal and spatial changes in periodograms (i.e. non-stationarity), and are described in the Supplement. For the temporal non-stationarity analysis, time series were constructed separately for each hour of the day, in part to suppress diel variability.

Sound pressure level (SPL) was calculated for each minute-long file obtained in the longterm recordings by band-pass filtering using a 4-pole Butterworth filter in 2 frequency bands: 100 to $1000 \mathrm{~Hz}$ and 2 to $20 \mathrm{kHz}$. These bands were chosen based on published frequency ranges of the majority of fish calls (Tricas and Boyle, 2014) and snapping shrimp sounds (Au and Banks, 1998). SPL was only calculated for DSG recordings from the same recording period as the DMONs and only for the DMON MF recordings, in order to match the DSG hydrophone frequency response more closely.

Abiotic noise, such as that produced by wind, can contribute substantially to SPL. In order to account for effects of wind speed, records were taken from a nearby weather buoy (National Data Buoy Center [NDBC] Station 41052, $18.251^{\circ} \mathrm{N}, 64.763^{\circ} \mathrm{W}$ ) and correlated with SPL in both frequency bands (averaged over the minute-long file length) for each recording and only for the time periods during which all 3 reefs had operational recorders. A similar analysis was conducted to characterize the relationship between temperature and SPL.

Octave band levels ( $\mathrm{dB}$ re $1 \mu \mathrm{Pa}$ ) were calculated for each clip with centroid frequencies $\left(F_{C}\right)$ at $125,250,500,1000$, and $2000 \mathrm{~Hz}$ (after Radford et al., 2014) using digital filters implemented in Matlab. Octave band levels were calculated for the new and full moon as follows. Lunar periodicity data were taken from the US Naval Observatory website (http://aa.usno.navy.mil/cgi-bin/aa_moonill2.pl). Data from $2 \mathrm{~d}$ on either side of 
the new and full moons that occurred during the deployment were denoted as 'new' or 'full' in both the fish and snapping shrimp bands and were combined for this analysis. Power spectra were produced for all clips using Welch's method (Welch, 1967). These power spectra were used to visually characterize changes in the spectral distribution of energy over time at each reef.

Acoustic entropy (H) index values (Sueur et al., 2008) were computed using the first 15 $\mathrm{s}$ of each of the resampled files. Entropy was computed in 3 bands using band-pass filters - the full band (100 to $20500 \mathrm{~Hz}$ ), the low-frequency fish band (100 to $1000 \mathrm{~Hz}$ ), and the high-frequency shrimp band (2 to $20 \mathrm{kHz}$ ). In addition, the Acoustic Complexity Index (ACI; (Pieretti et al., 2011) was computed for each file using a temporal step of $12 \mathrm{~s}$ and FFT size of 880 points. The Bonferroni correction was used to reduce the critical p-value whenever multiple comparisons were carried out.

\subsection{Results}

Coral cover differed significantly among reefs (Figure 2.2A, $\mathrm{F}_{2,23}=19.24, \mathrm{p}<0.001$ ) and the results of a randomization test indicated that the probability of getting this result by chance was less than 1 in 1000. Coral cover was highest at Tektite. Ram Head was lowest in coral cover but highest in 'Other' (which included crustose coralline algae, turf algae, other invertebrates, bare rock, and sand). Fish assemblages also differed significantly among reefs (Figure 2.2B, $\chi^{2}=26.573, \mathrm{df}=2, \mathrm{p}<0.001$ ); however, the results of a randomization test indicated that the probability of this result arising by chance was greater than 0.05 . Observed herbivore and consumer fish densities were highest at Tektite and lowest at Ram Head, with no difference in invertivore density.

Results from the spatial non-stationarity analysis (see the Supplement for methods) indicated significant differences within reefs among recorders over the $24 \mathrm{~h}$ short-term deployments (Tektite: $\chi^{2}=3.99 \times 10^{5}, \mathrm{df}=3.1 \times 10^{5}, \mathrm{p}<0.0001$; Yawzi: $\chi^{2}=4.02 \times 10^{5}, \mathrm{df}=$

$3.1 \times 10^{5}, \mathrm{p}<0.0001$; Ram Head: $\left.\chi^{2}=4.15 \times 10^{5}, \mathrm{df}=3.3 \times 10^{5}, \mathrm{p}<0.0001\right)$. However, these differences appeared to be very small (Figure 2.3). While there was some variability among recorders at any one frequency, all power spectra followed a similar shape at a given time and reef. Thus, statistical differences were likely a result of high statistical power, and for all further analyses data from a single recorder was used. 


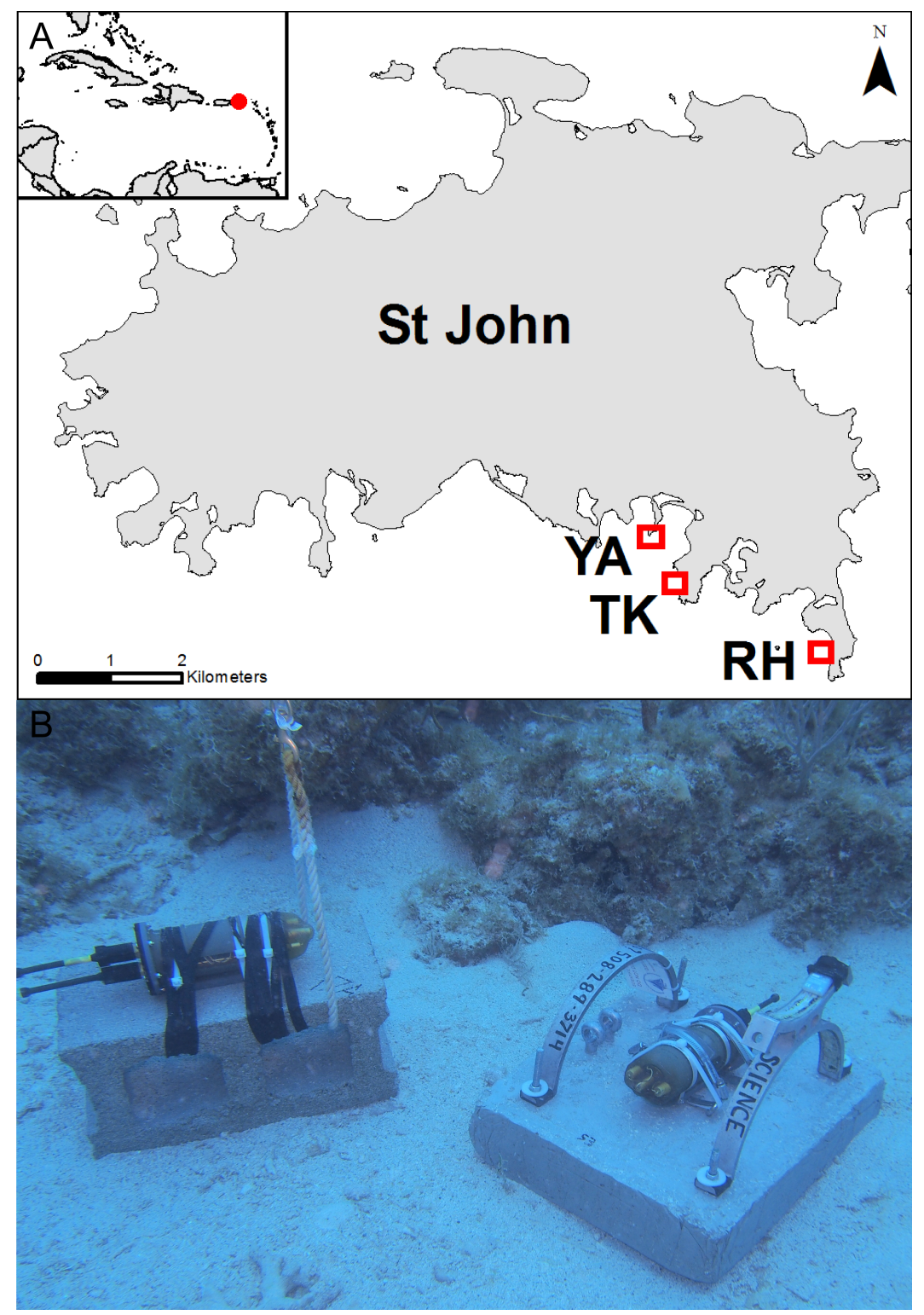

Figure 2.1: (A) Locations of 3 reefs located within the U.S. Virgin Islands National Park on which acoustic recording devices were deployed (TK: Tektite, YA: Yawzi, RH: Ram Head); lat/long: $18.336^{\circ} \mathrm{N}, 64.739^{\circ} \mathrm{W}$ ). (B) Example of acoustic recorder moorings showing a DMON deployed for (left) a $24 \mathrm{~h}$ period and (right) a 4 mo duty-cycled period. 

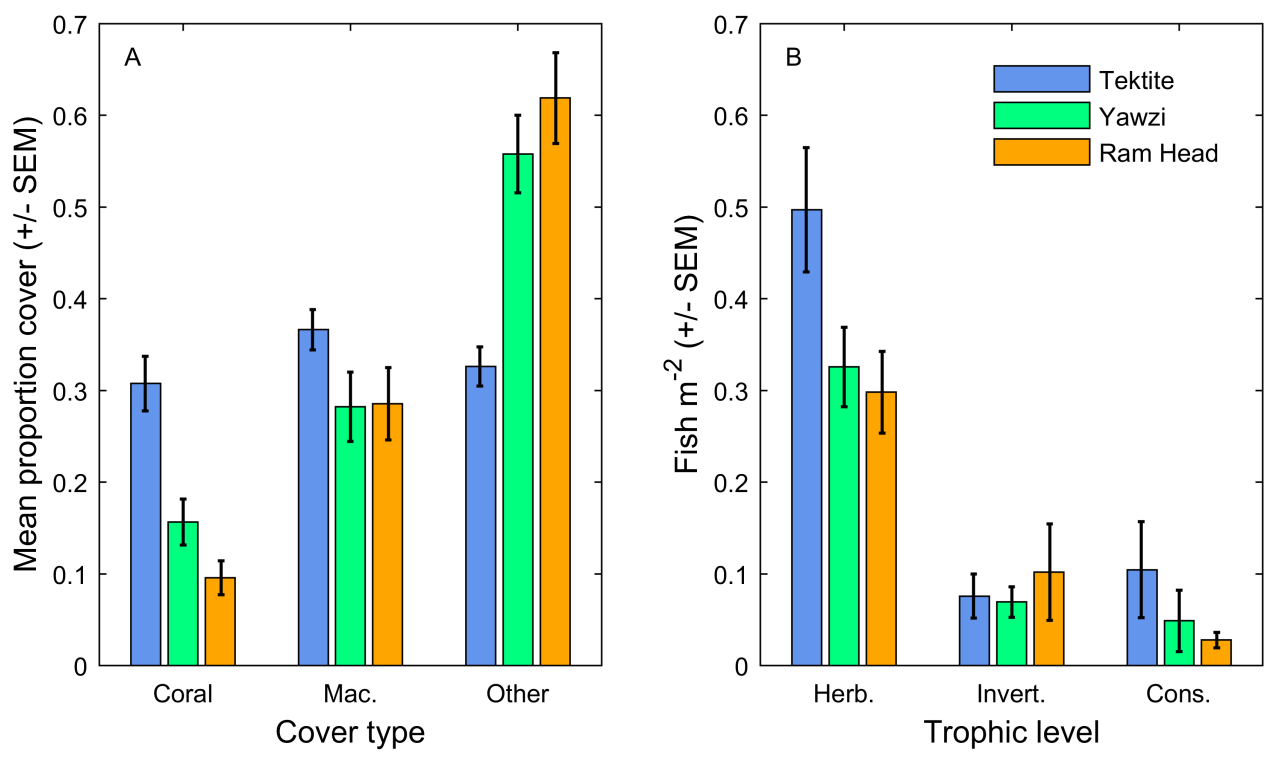

Figure 2.2: (A) Proportion (mean \pm SEM) of benthic cover (coral, macroalgal, and other) and (B) fish density by trophic level (mean density $m^{2} \pm \mathrm{SEM}$ ) for each reef. Fish density was split into functional groups (herbivores, invertivores, and consumers).

Median sound pressure levels in the low-frequency fish band were typically highest at Tektite and differences among reefs were most pronounced at approximately dusk and dawn (Figure 2.4A). At other times, the differences were often marginal. Ram Head typically showed the lowest median sound pressure, with considerably reduced levels during the day, and only slightly reduced levels compared to the other reefs at night. The median SPL in the high-frequency shrimp band was greatest at Yawzi, followed by Ram Head and then Tektite (Figure 2.4B). Elevated median sound production was apparent at dawn but not at dusk. Unlike in the fish band, the shrimp band seemed to follow a similar daily pattern at all reefs with differences mainly in sound intensity.

The magnitude of the diel trend in low-frequency fish sounds (i.e., the difference between median low-frequency SPL at dusk [ca. 18:00 h] and midnight, and dawn [ca. 06:00 h] and midnight) was significantly but weakly correlated with percent coral cover and fish density (Table 2.2), with the strongest trend apparent at Tektite, followed by Yawzi and Ram Head (Figure 2.5). There was only 1 significant correlation in the high-frequency snapping shrimp band (between the strength of the dawn peak in sound production and fish density). This correlation was weaker than all of the correlations between trends in low-frequency fish-sound production and species assemblages. 


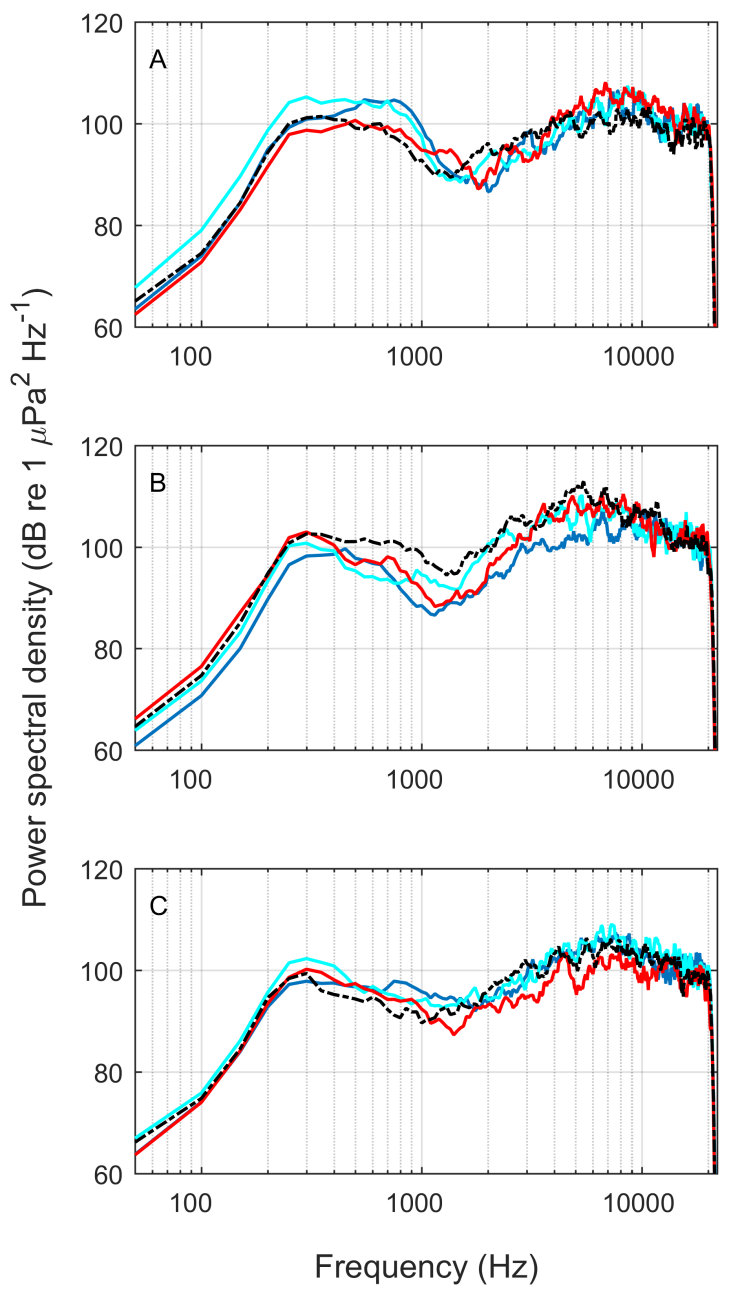

Figure 2.3: Power spectra from each of 4 recorders (different colors) at dusk (18:00 h) for (A) Tektite, (B) Yawzi, and (C) Ram Head. Recordings were collected over $24 \mathrm{~h}$ periods in August 2013. Power spectra have been smoothed using convolution. The shapes of the spectra at a given reef and time of day are similar, with some variation among recorders at certain frequencies.

Temperature increased over the deployment period across all 3 reefs from ca. $26.7^{\circ} \mathrm{C}$ in mid-April to ca. $29.4^{\circ} \mathrm{C}$ in early August, with limited variation among reefs. There were no significant correlations between temperature and SPL in the fish band at any of the 3 reefs. Correlations in the shrimp band were significant at both Tektite and Ram Head but not Yawzi (Tektite: $R^{2}=0.025, F_{1,720}=18.273, \mathrm{p}<0.0001$; Ram Head: $R^{2}=0.07, F_{1,819}$ $=58.261, \mathrm{p}<0.0001$; Yawzi: $R^{2}=0.001, F_{1,836}=0.815, \mathrm{p}=$ not significant $)$. Wind speed was not significantly correlated with SPL in either frequency band at any site except for 

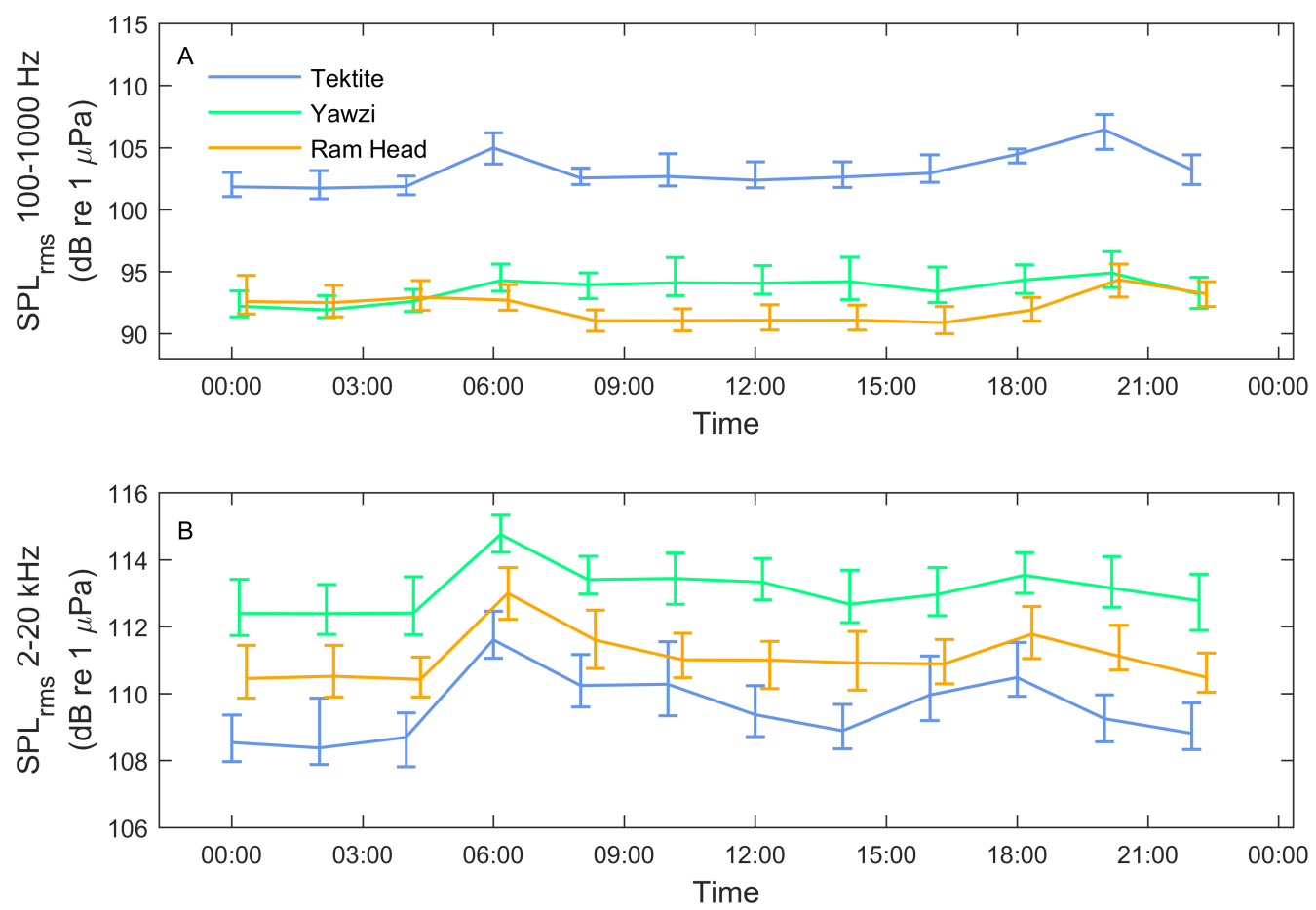

Figure 2.4: Sound pressure level (SPL, rms) in the (A) 100 to $1000 \mathrm{~Hz}$ band and (B) 2 to $20 \mathrm{kHz}$ band, pooled across a ca. 4 mo recording duration at each reef (median with 25-75 percentiles). The greatest peaks in SPL in the low-frequency fish band (A) were at Tektite at dawn $(06: 00 \mathrm{~h})$ and after dusk $(20: 00 \mathrm{~h})$, with smaller peaks at Yawzi, and no observable peaks at Ram Head. Overall high-frequency SPL (B) was highest at Yawzi, followed by Ram Head and then Tektite for all times of day, with a stronger peak at dawn than dusk at all 3 reefs.

Ram Head in the shrimp band $\left(R^{2}=0.03, F_{1,756}=21.668, \mathrm{p}<0.0001\right.$, see Figure 2.S1 in the Supplement).

The low-frequency fish band was separated into octaves $(125,250,500,1000,2000 \mathrm{~Hz})$ to identify the frequencies at which sound levels were elevated. While the distribution of energy did not differ consistently among sites or between the new or full moon, there were differences at certain frequencies. In particular, octave band levels were elevated at $500 \mathrm{~Hz}$ at Tektite at dusk and dawn compared to other reefs during the new and full moon (Figure 2.6). Octave band levels were elevated at $2000 \mathrm{~Hz}$ for all times of day at Yawzi and Ram Head, likely as a result of higher shrimp snapping amplitudes at those reefs compared to Tektite.

Power spectral density estimates were combined to produce heat maps showing changes 


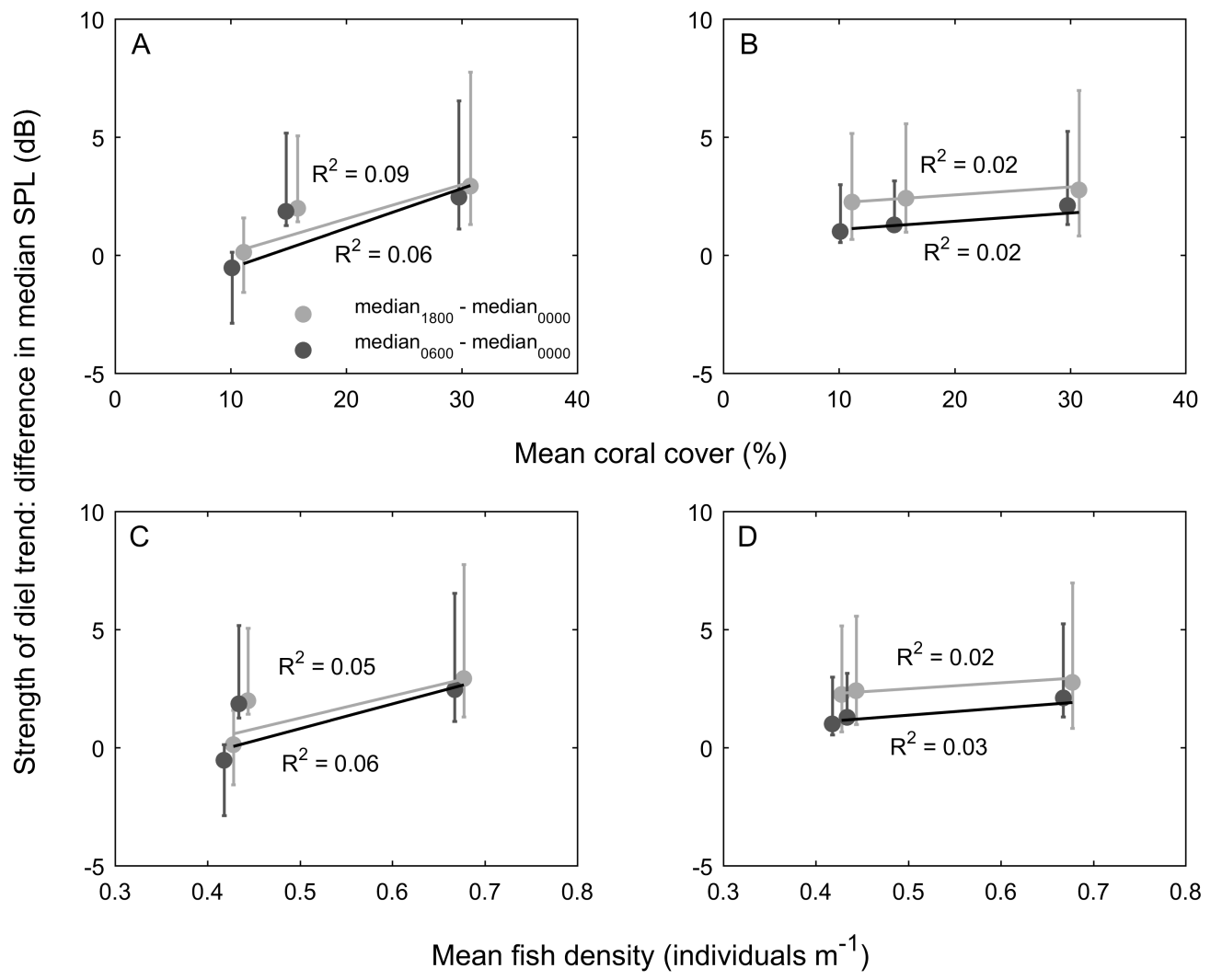

Figure 2.5: Correlation between $(\mathrm{A}, \mathrm{B})$ mean coral cover and $(\mathrm{C}, \mathrm{D})$ mean fish density at each reef and the strength of diel trend, measured as the difference in median sound pressure level (SPL) between dusk (ca. 18:00 h) and midnight, and dawn (ca. 06:00 h) and midnight, in $(\mathrm{A}, \mathrm{C})$ the 100 to $1000 \mathrm{~Hz}$ and (B,D) the 2 to $20 \mathrm{kHz}$ bands over the 4 mo duty-cycled recording period (differences shown with $25-75$ percentiles). $R^{2}$ values are shown adjacent to the linear regressions.

in the distribution of energy by frequency over a $24 \mathrm{~h}$ period for each site in July 2013 (Figure 2.7). These visual representations indicate that spectral and temporal variability was greatest at Tektite, with diel trends of sound increases at dusk and dawn. The crepuscular trend was present but difficult to discern for Yawzi, and largely absent for Ram Head. The noon peak at Ram Head was a result of some incidental (likely anthropogenic) impulse noise that was recorded, reflecting the intrusion of abiotic sounds into the coral reef soundscape.

Results from the temporal non-stationarity analysis indicated that power spectra for each reef changed over the deployment period (Tektite: $\chi^{2}=1.56 \times 10^{7}$, df $=3.7 \times 10^{5}, \mathrm{p}<$ 0.0001; Yawzi: $\chi^{2}=1.94 \times 10^{7}, \mathrm{df}=3.7 \times 10^{5}, \mathrm{p}<0.0001$; Ram Head: $\chi^{2}=1.92 \times 10^{7}, \mathrm{df}$ $\left.=3.7 \times 10^{5}, \mathrm{p}<0.0001\right)$. Results were similar when this analysis was carried out separately 

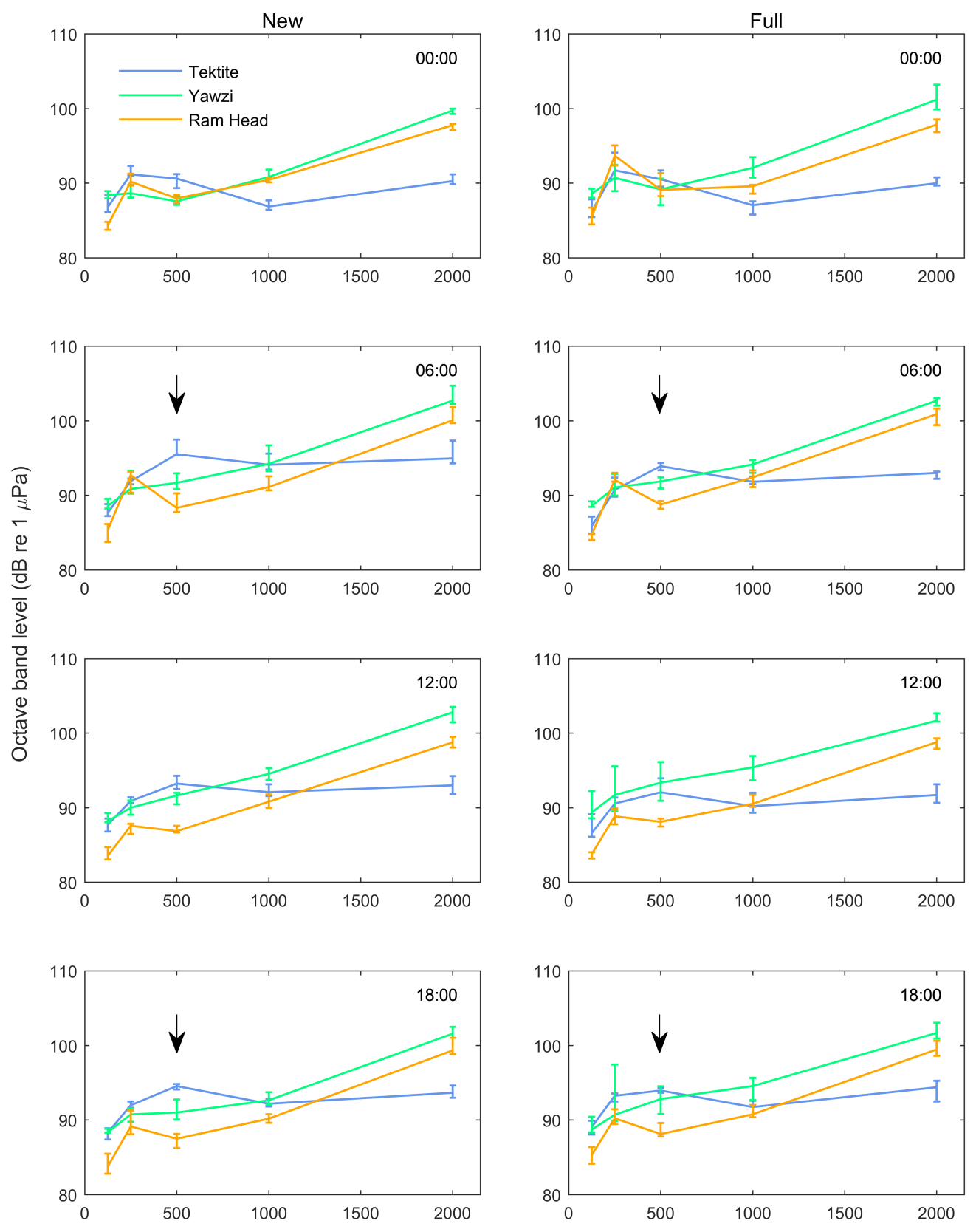

Octave band $(\mathrm{Hz})$

Figure 2.6: Octave band levels $\left(F_{C}=125,250,500,1000,2000\right)$ at 4 times of day (00:00, 06:00, 12:00, 18:00 h) at the new and full moons, pooled over the 4 mo recording period (median with $25-75$ percentiles). low-frequency octave band levels $(500 \mathrm{~Hz})$ are elevated at Tektite compared to other sites at dusk and dawn during the new and full moon (black arrows).

for each time of day (i.e., power spectra for each time of day changed over the course of the deployment). However, there was no clear trend over time; rather, spectra varied on short time scales throughout the deployment period within each reef (Figure 2.8). 
Table 2.2: Statistical results from the correlation of the strength of diel trends in sound production in low-frequency (LF) and high-frequency (HF) at dawn and dusk with coral cover and fish density from each reef. Alpha was reduced using the Bonferroni correction to 0.0063 .

\begin{tabular}{l|l|l|l|l|l|l} 
& \multicolumn{3}{|c|}{ Coral cover } & \multicolumn{3}{c}{ Fish density } \\
\hline & $R^{2}$ & $F_{1,236}$ & $\mathrm{p}$ & $R^{2}$ & $F_{1,236}$ & $\mathrm{p}$ \\
\hline LF-dawn & 0.087 & 22.4 & $<0.001$ & $0.062-2$ & 15.642 & $<0.001$ \\
LF-dusk & 0.060 & 15.125 & $<0.001$ & 0.046 & 11.535 & $<0.001$ \\
HF-dawn & 0.023 & 5.458 & $0.02-$ & 0.031 & 6.53 & 0.006 \\
HF-dusk & 0.024 & 5.773 & 0.017 & 0.025 & 6.075 & 0.014
\end{tabular}

To test what kind of sounds drive the acoustic entropy index, we partitioned the entropy into both fish and shrimp bands and compared them to the full band entropy. Acoustic entropy values were largely driven by the 2 to $20 \mathrm{kHz}$ (shrimp) frequency range (Figure 2.9). Correlations between entropy calculated in just the shrimp band and in the full band were strong (Figure 2.9A2) or very strong (Figure 2.9B2,C2) for all 3 reefs. However, correlations between entropy in the fish band and the total band were very weak at all 3 reefs. There were significant differences among reefs in the mean entropy values in the fish band $\left(F_{2,3068}=\right.$ 151.56, $\mathrm{p}<0.0001$ ), and the results of a post hoc test indicated that the fish entropy values at Yawzi and Ram Head were significantly higher than those at Tektite. This result is divergent from the visual survey data and the finding that the strength of the diel trend in sound production in the fish band correlates with coral cover and fish density, as fish band entropy did not correlate with either of these biological attributes of the reefs.

Mean ACI values (Tektite: 341.4; Yawzi: 355.1; Ram Head: 354.2) did not follow a discernable pattern and were not correlated with species assemblages at each reef. Similarly, the diel trend in ACI values did not correlate with the diel trend in SPL.

\subsection{Discussion}

Marine soundscapes are increasingly being studied as a means to identify differences among habitats. This is the first study, to our knowledge, that pairs a detailed assessment of the soundscape with traditional visual surveys of coral reef benthos and fish communities. The goals were to both evaluate the spatiotemporal variation of the soundscapes of 3 reefs and address how these differing acoustic environments may be representative of different habitats or community structure. Acoustic differences among reefs were evident but were 

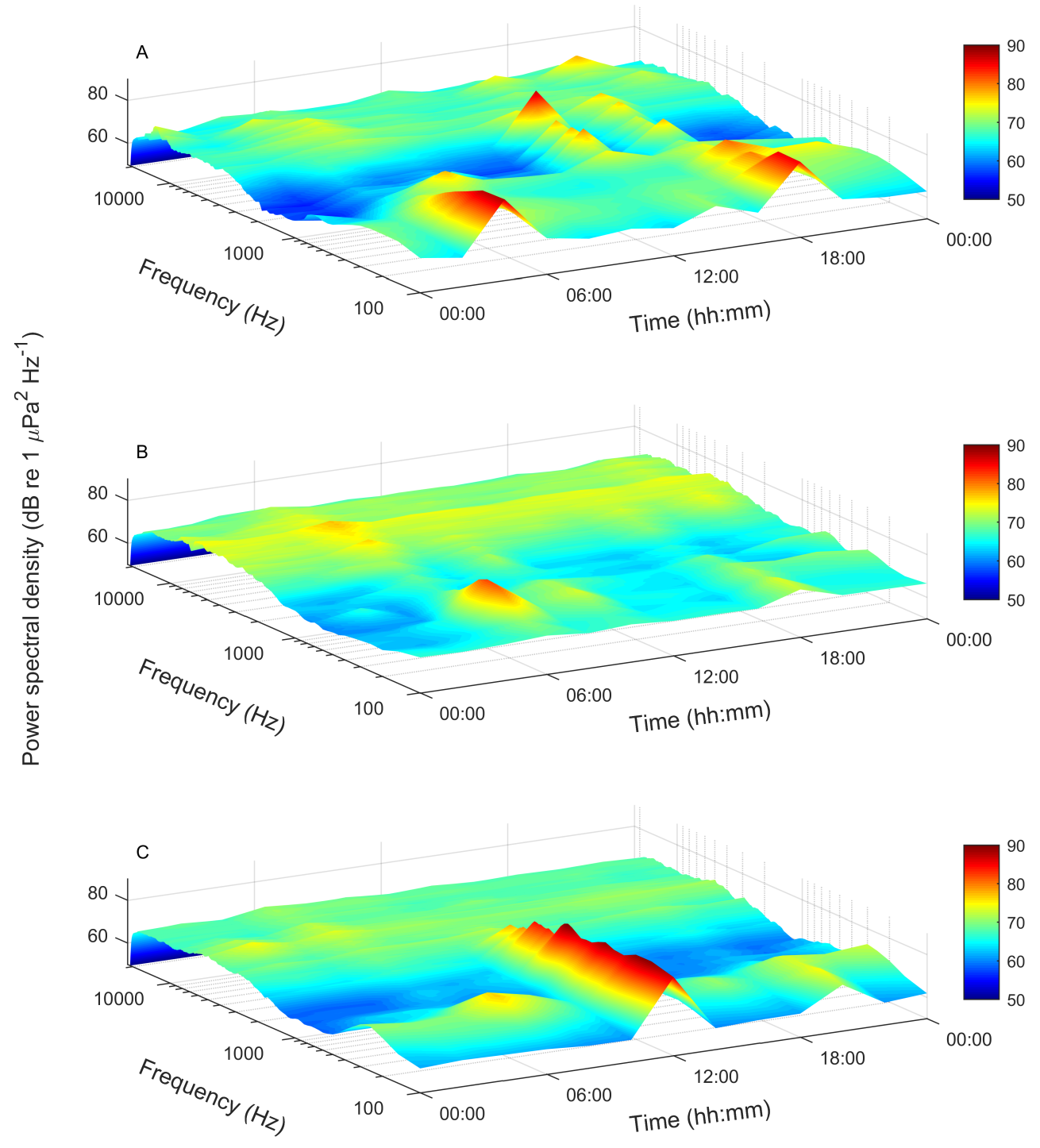

Figure 2.7: Power spectral density estimates over a $24 \mathrm{~h}$ period from 4 to 5 July 2013 at (A) Tektite, (B) Yawzi, and (C) Ram Head, displayed up to $20 \mathrm{kHz}$. Color bars indicate power spectral density. Peaks in sound production are apparent at Tektite at dusk and dawn. Smaller peaks are apparent at dusk at Yawzi and Ram Head, with less energy at dusk. This period of time was exclusive of boat noise at all reefs but a series of 5 consecutive high-amplitude impulsive sounds is the cause of the elevated energy at noon at Ram Head.

relatively small in magnitude. For example, there were differences between sites in the low-frequency fish band at crepuscular times, when fish and invertebrates may be more acoustically active (Lammers et al., 2008; Radford et al., 2014; Staaterman et al., 2014), 
but not at many other times of day. These differences at low frequencies were associated with similar but also relatively small differences in coral cover and fish density. While there were significant differences in fish assemblages among reefs, these were not sufficiently large enough in magnitude to withstand a randomization test. The reduced SPL during the day in the fish band at Ram Head but not at the other reefs could result from among-reef differences in the acoustically active fishes. Subsequent investigations should attempt to identify the individual sound types present at each reef to better address this question. Overall, results suggest that the species assemblages present at each reef were associated with the localized bioacoustics soundscape. However, data collected from reefs that vary more in fish density and coral cover would likely better elucidate this trend. The regression-based methods used here (Figure 2.5) suggest that there are soundscape gradients relative to some measures of community structure. While more reefs were evaluated here than in other studies (e.g., Staaterman et al., 2013, 2014), investigations that address additional reefs could provide further data on these links.

Notably, there were fewer differences between sites and soundscapes in the high-frequency snapping shrimp band. While overall shrimp sound pressure levels varied in intensity, the temporal trends did not differ between reefs of varying species assemblages (Figure 2.5). This suggests that while shrimp were potentially more abundant at Yawzi, their snapping patterns followed the same diel trends. This is perhaps reflective of the relative similarity of physical conditions between sites (e.g., light, depth, and temperature). Snapping shrimp patterns were not related to coral cover (Figure 2.6), which suggests that this portion of the soundscape, while often dominant in intensity, may not be reflective of benthic cover and associated fish assemblages. Indeed, snapping shrimp sounds are abundant in a variety of habitats (Lammers et al. 2008). Unlike many fish species, snapping shrimp are not similarly reliant on live coral for shelter and nutrition; instead they select habitat that provides shelter such as rocky outcrops, live or dead coral, and sponges (Cato and Bell, 1992). Accordingly, their abundance (and by extension, sound production) are likely not driven by live coral cover per se, which could explain the lack of relationship between reef coral cover and shrimp sound production. If coral reef sounds are used for settlement, this discrepancy suggests that the higher-frequency snapping shrimp band may be a less reliable indicator of suitable habitat.

Results from the short-term deployment indicated that there were significant spectral 
differences among recorders on a reef at a given time of day; however, a visual assessment of a subset of these spectra confirms only limited inter-recorder variability (Figure 2.3). The biological relevance of these small differences is unknown. Animals may be able to discern these finescale acoustic differences when searching for suitable settlement habitat, but given the overall consistency in power spectra within reefs, it is likely that a single sensor deployed on a reef would be adequate to record the broad trends in sound production.

The presence of diel trends in both frequency bands suggests that snapshot-type recordings likely miss substantial temporal trends. Given that differences among reefs may only be evident when sound production increases (i.e., during crepuscular times), these results reflect the importance of collecting recordings over at least a diel cycle to capture these natural trends. For example, a recording collected at noon would not necessarily indicate any difference in sound production at low-frequency between Tektite and Yawzi, whereas a recording collected at dusk would (Figure 2.4).

There was considerable variability in power spectral density at $500 \mathrm{~Hz}$ and $5 \mathrm{kHz}$ at each reef over the course of the deployments (Figure 2.8), but these changes were not associated with the temporal or physical characteristics measured (lunar cycles, changes in temperature or wind speed). Because of this variability within reefs over time, recordings collected over longer durations (i.e., weeks to months) may be best suited to characterizing trends in sound production and for comparing among reefs.

Coral cover and reef architectural complexity play a major role in influencing fish assemblages (e.g., McCormick, 1994; Friedlander et al., 2003; Jones et al., 2004). Thus, the greater magnitude trend in low-frequency diel sound production at Tektite may be driven by links between elevated coral cover and higher fish densities at that reef. As many fish species produce sound during spawning, which often occurs around the new moon (D'Alessandro et al., 2007), elevated levels of low-frequency SPL at Tektite during the new moon could be linked to spawning activity, which has been shown to occur around the time of year that these instruments were deployed (D'Alessandro et al., 2007).

Several behavioral experiments have suggested that larval fish may use reef sound to identify a suitable settlement habitat (e.g., Tolimieri et al., 2000, 2004; Simpson et al., 2004, 2005; Radford et al., 2011a). Most fishes without specialized morphological adaptations cannot hear frequencies higher than around $1000 \mathrm{~Hz}$ (Popper and Fay, 2011), and the majority of fish calls are often below $1000 \mathrm{~Hz}$ (Tricas and Boyle, 2014). Thus, low-frequency sounds 

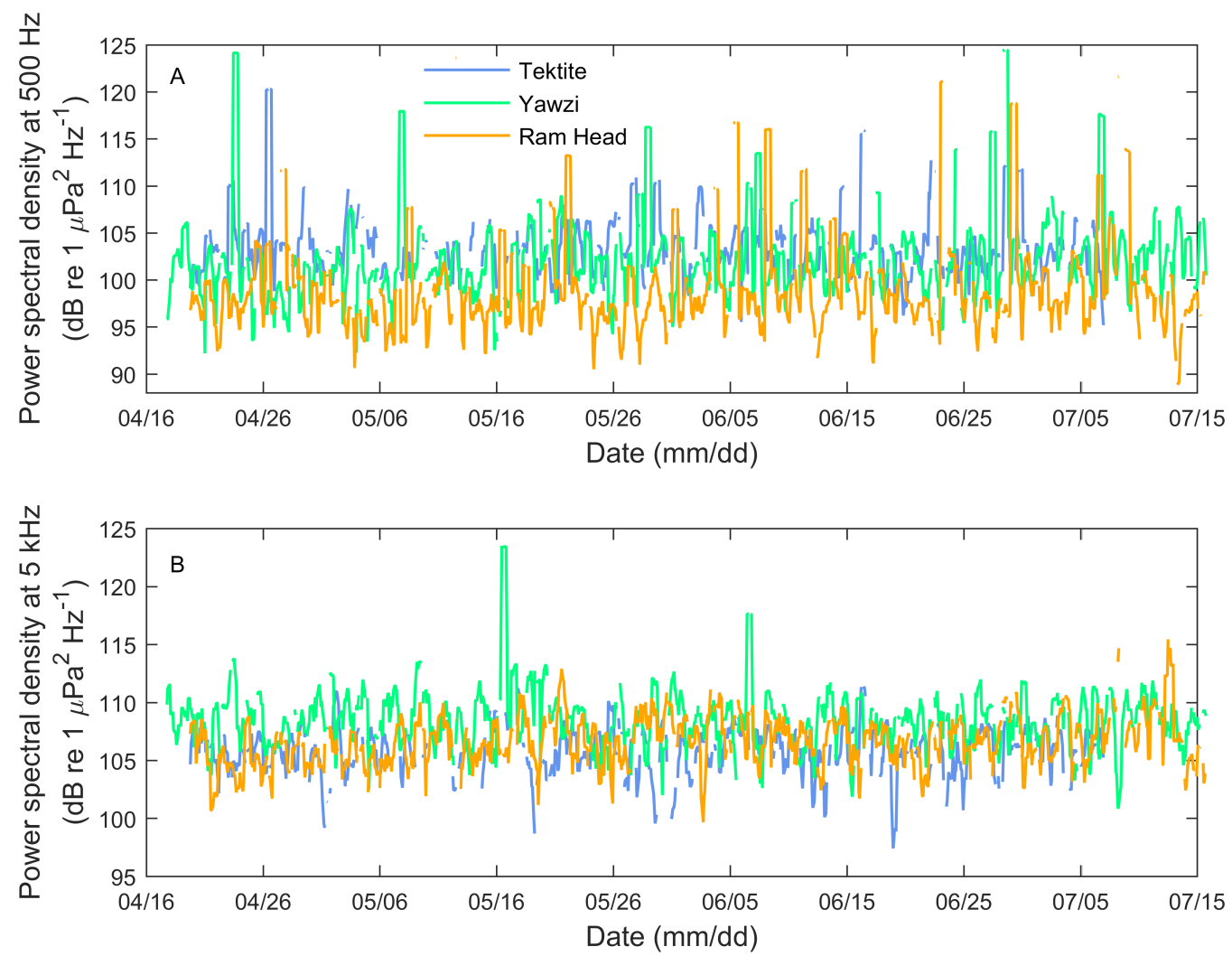

Figure 2.8: Power spectral density at (A) $500 \mathrm{~Hz}$ and (B) $5 \mathrm{kHz}$ for 3 reefs from the entire long-term deployment period. Missing data (white space) is a result of files containing boat noise being removed and/or recorder failure (e.g., end of blue line). Results from the non-stationarity analysis indicate that the periodograms changed over the course of the deployment period; however, there is no clear temporal trend at any site. Rather, there is constant variability.

on reefs will likely be of higher relevance as a settlement cue than high frequencies (cf. Simpson et al., 2008), where sound production may not be as closely linked to habitat quality. Accordingly, passive acoustic recordings of these lower-frequency fish bands may provide a better evaluation of community assemblages. Ocean noise levels are increasing at low frequencies as a result of commercial shipping activities (Andrew et al., 2011; McDonald et al., 2006; Chapman and Price, 2011). While most of these increases are below $300 \mathrm{~Hz}$, an elevated low-frequency noise floor could have adverse effects on acoustic communication in fishes and, by extension, larval recruitment to reefs.

If differences in low-frequency sound production were a result of differences in fish species assemblages, why was only limited difference in fish density detected through the visual 

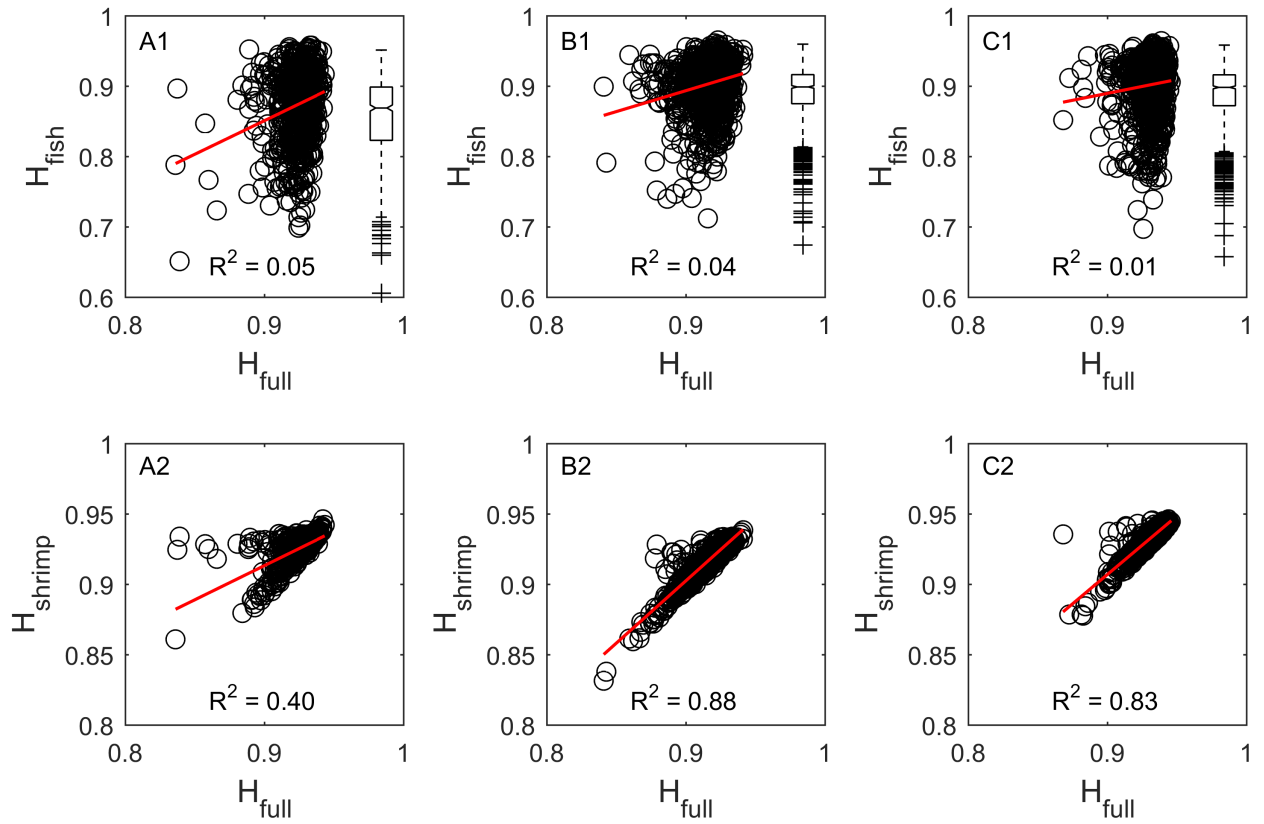

Figure 2.9: Top graphs: relationships between entropy $(\mathrm{H})$ calculated in a low-frequency fish band $(100$ to $1000 \mathrm{~Hz})$ and the full band $(100 \mathrm{~Hz}$ to $20 \mathrm{kHz})$ and boxplots of the range of lowfrequency $\mathrm{H}$ at (A1) Tektite, (B1) Yawzi, and (C1) Ram Head (central mark is the median and the edges of the box are 25th and 75th percentiles). Bottom graphs: the relationship between $\mathrm{H}$ in a high-frequency shrimp band (2 to $20 \mathrm{kHz})$ and the full band at each of the 3 sites (A2, B2, C2). Linear regressions are shown in red and $R^{2}$ values are included for each relationship.

surveys? Many soniferous species, such as squirrelfishes (Amorim, 2006), are cryptic and nocturnal. Other species may also shy away from divers and human observers. Thus, visual fish surveys conducted during daylight hours may largely miss these and similar species (Brock, 1982). As far as possible, further studies should ensure that efforts are taken to identify cryptic species, which may disproportionately contribute to acoustic recordings. Similar analyses may also benefit from comparing reefs with larger differences in fish assemblages. Acoustic data have the benefit of recording fishes that may be missed by visual surveys; however, the number of soniferous fishes is not well known. Out of approximately 30000 extant fish species, 700 are known to produce sound across at least 30 families (Luczkovich et al., 2008). Additional effort to determine the distribution of sound production across unstudied fishes is needed.

Acoustic entropy values were largely driven by snapping shrimp activity. Shrimp snaps are broadband and were ubiquitous in the acoustic records obtained at all 3 reefs. How- 
ever, they represent a single sound type. Accordingly, high values of acoustic entropy from snapping shrimp sounds are not reflective of overall acoustic diversity. To address this fundamental limitation of this index, we compared the entropy calculated in a low-frequency fish band to visual survey data and observed trends in amplitude at each reef, but no relationship was observed. While it is possible that this index is correctly identifying Yawzi as the reef with highest call diversity, that finding is not supported by either the visual survey data or the sound amplitude data.

Similarly, the ACI did not yield results that were consistent with the other analyses presented here. There are a few reasons why this might be the case. ACI values could be higher in recordings with lots of variability (i.e., large amplitude differences between adjacent frequency and time bins) but this variability could result from a small number of vocalizations spaced throughout a recording. Conversely, in a soundscape with high bioacoustic activity, ACI values could be low because of a high density of calling activity (i.e., chorusing) and corresponding small differences in intensity between frequency and time bins. Thus, more work is needed to develop an index that is well suited to marine soundscapes.

\subsection{Conclusion}

Assessments of extended acoustic records from reefs that vary in species assemblages reflect trends in biological sound production that correlate with coral cover and fish density. In particular, low-frequency sound, which is dominated by fish calls, and crepuscular soniferous activity, when fish are most acoustically active, provided the strongest links to visual characterizations of the reef. Conversely, high-frequency acoustic recordings of snapping shrimp did not correlate with the species present. Acoustic and biological differences among reefs were small, and further work on a broader range of reefs is needed to better elucidate these

relationships. Additional work is also needed to develop acoustic diversity indices that are suitable for the marine environment. Overall, these results strengthen the basis for using acoustic recordings to help guide monitoring and conservation efforts. 


\section{7 $\quad$ Supplementary Material}

\subsubsection{Supplementary figure}

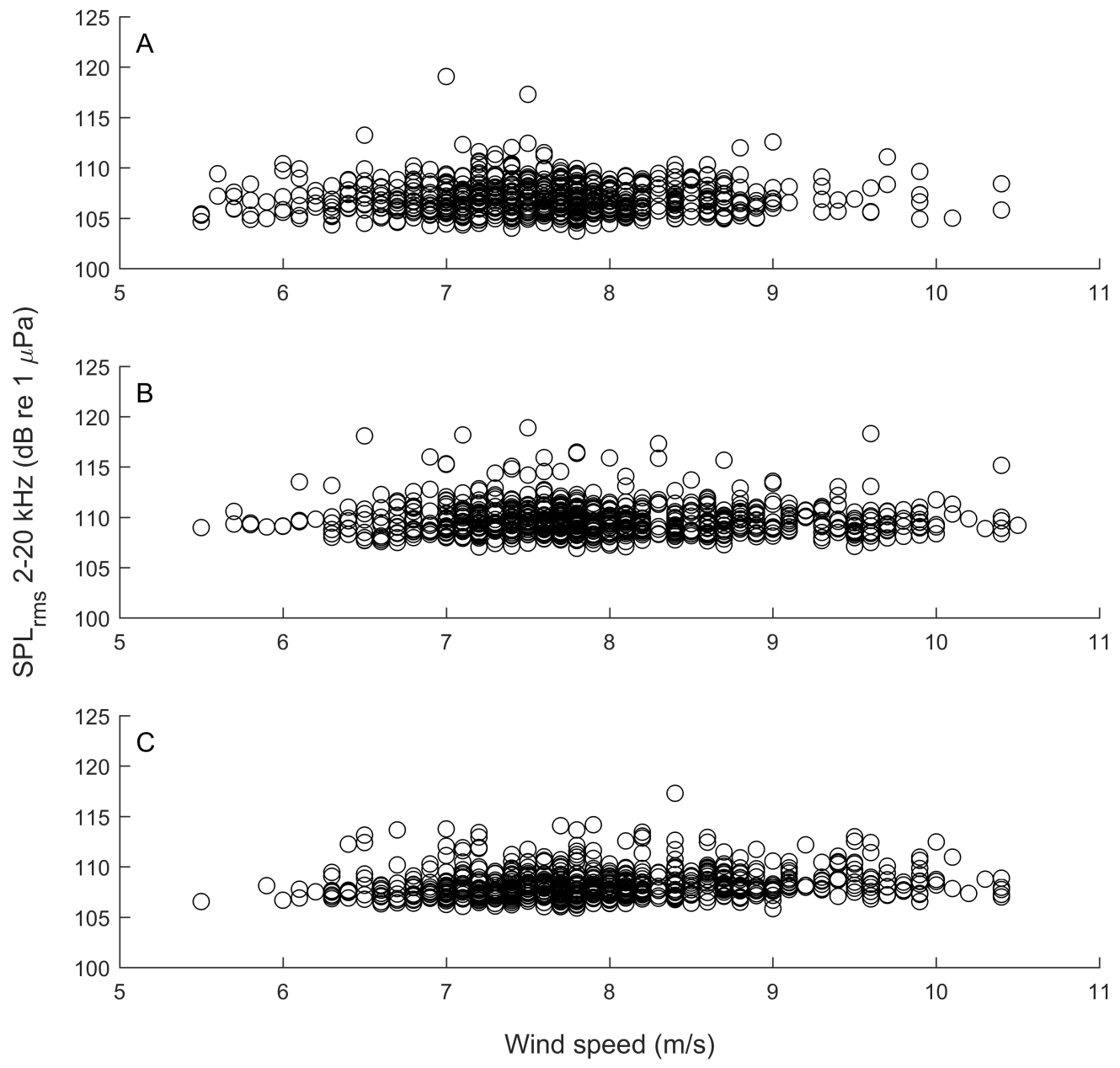

Figure 2.S1: Relationship between sound pressure level in the high-frequency shrimp band $(2-20 \mathrm{kHz})$ and wind speed for three U.S. Virgin Islands reefs, Tektite (A), Yawzi (B), and Ram Head (C). Only Ram Head demonstrated a significant relationship between wind speed and sound pressure level, albeit weakly. 


\subsubsection{Non-stationarity analysis}

This supplement outlines periodogram-based likelihood ratio (LR) tests for temporal and spatial non-stationarity of the spectral density function (sdf) of reef sound. The statistical results underlying these tests are available in Dzhaparidze (1986).

Let $X_{t h}, t=1,2, \ldots, T$, be a collection of $T$ independent time series each of length $n$ recorded at times of day $h=1,2, \ldots, H$ on days $t=1,2, \ldots, T$ and let $f_{t h}(\omega)$ be the unknown sdf at time of day $h$ on day $t$. Interest centers on testing the null hypothesis $H_{o}$ : $f_{t h}(\omega)=f_{h}(\omega)$ for all $h$ and $t$ that the sdf at each time of day is stationary over time against the general alternative hypothesis $H_{1}$ that it is not.

Let $I_{t h}\left(\omega_{j}\right)$ be the periodogram ordinate for $X_{t h}$ at Fourier frequency $\omega_{j}, j=1,2, \ldots, J$. It is a standard result that $I_{t h}\left(\omega_{j}\right)$ is approximately independent of $I_{t h}\left(\omega_{k}\right)$ and has an approximate exponential distribution with mean $f_{t h}\left(\omega_{j}\right)$ and probability density function:

$$
g\left(I_{t h}\left(\omega_{j}\right)\right)=\frac{1}{f_{t h}\left(\omega_{j}\right)} \exp \left(-\frac{I_{t h}\left(\omega_{j}\right)}{f_{t h}\left(\omega_{j}\right)}\right)
$$

The log likelihood is given by:

$$
\log L=-\sum_{t=1}^{T} \sum_{h=1}^{H} \sum_{j=1}^{J}\left(\log f_{t h}\left(\omega_{j}\right)+\frac{I_{t h}\left(\omega_{j}\right)}{f_{t h}\left(\omega_{j}\right)}\right)
$$

The maximum likelihood (ML) estimate of $f_{t h}\left(\omega_{j}\right)$ under $H_{1}$ is simply $I_{t h}\left(\omega_{j}\right)$ and the corresponding maximized value of the log likelihood is:

$$
\log L_{1}=-T H J-\sum_{t=1}^{T} \sum_{h=1}^{H} \sum_{j=1}^{J} \log I_{t h}\left(\omega_{j}\right)
$$

The ML estimate of the common sdf $f_{h}\left(\omega_{j}\right)$ under $H_{o}$ is the periodogram average:

$$
\hat{f}_{h}\left(\omega_{j}\right)=\frac{1}{T} \sum_{t=1}^{T} I_{t h}\left(\omega_{j}\right)
$$

and the corresponding maximized value of the log likelihood is:

$$
\log L_{o}=-T H J-T \sum_{h=1}^{H} \sum_{j=1}^{J} \log \hat{f}_{h}\left(\omega_{j}\right)
$$

Finally, the LR statistic for testing $H_{o}$ against $H_{1}$ is: 


$$
L R=2\left(\log L_{1}=\log L_{o}\right)
$$

which, under $H_{o}$, has an approximate chi squared distribution with degrees of freedom given by $(T-1) H J$.

The same general approach can be used to test for spatial non-stationarity. Let $X_{t k}, t=$ $1,2, \ldots, T$ be a collection of $T$ independent time series each of length $n$ recorded at locations $k=$ $1,2, \ldots, K$ at times $t=1,2, \ldots, T$ and let $f_{t k}\left(\omega_{j}\right)$ be the unknown sdf at locationk at time $t$. Interest centers on testing the null hypothesis $H_{o}: f_{t k}\left(\omega_{j}\right)=c_{t k} f_{t}\left(\omega_{j}\right)$ that at each time the sdf's at the different locations are the same up to a multiplicative scaling against the general alternative hypothesis $H_{1}$ that they are not. For definiteness, under $H_{o}$, take $c_{t 1}=1$ for all $t$.

As before, the maximized value of the log likelihood under $H_{1}$ is:

$$
\log L_{1}=-T K J-\sum_{t=1}^{T} \sum_{k=1}^{K} \sum_{j=1}^{J} \log I_{t k}\left(\omega_{j}\right)
$$

where $I_{t k}\left(\omega_{j}\right)$ is the value of the peridogram at time $t$ and location $k$ for Fourier frequency $\omega_{j}$. Maximizing the $\log$ likelihood under $H_{o}$ must be done numerically. In doing so, it is helpful to note that for fixed values of the scaling parameters $c_{1}=1, c_{2}, \ldots, c_{K}$, the ML estimate of $f_{t}\left(\omega_{j}\right)$ is the weighted average:

$$
\hat{f}_{t}\left(\omega_{j}\right)=\frac{1}{K} \sum_{j=1}^{J}\left(I_{t k}\left(\omega_{j}\right) / c_{k}\right)
$$

As before, the LR statistic for testing $H_{o}$ against $H_{1}$ is:

$$
L R=2\left(\log L_{1}=\log L_{o}\right)
$$

where $\log L_{o}$ is the numerically maximized $\log$ likelihood under $H_{o}$. Under $H_{o}$, LR has an approximate chi squared distribution with degrees of freedom given by $T(K J-(K+J-1))$. 
THIS PAGE INTENTIONALLY LEFT BLANK 


\section{Chapter 3}

\section{Acoustic and biological trends on coral reefs off Maui, Hawaii}

This chapter is currently in preparation for submission as: Kaplan, M.B., Lammers, M.O., Zang, E., Solow, A.R., \& Mooney, T.A. Acoustic and biological trends on coral reefs off Maui, Hawaii. 


\subsection{Abstract}

Coral reef soundscapes are dynamic and demonstrate amplitude and spectral variability across timescales from seconds to seasons. This biological sound production may reflect the appreciable biodiversity of these ecosystems. To investigate how sounds produced on a given reef relate to abiotic and biotic parameters and how that relationship may change over time, an observational study was started in September 2014. Seven Hawaiian reefs that vary in coral cover, rugosity, and fish assemblages were equipped with temperature loggers and acoustic recording devices that recorded on a $10 \%$ duty cycle for 16 months. Benthic and fish visual survey data were collected 4 times over the course of the study. On average, reefs ranged from 0 to $80 \%$ live coral cover, although changes between surveys were noted, in particular during the major El Nino-related bleaching event of October 2015. Acoustic analyses were carried out in 2 frequency bands $(50-1200 \mathrm{~Hz}$ and $1.8-20.5 \mathrm{kHz})$ that corresponded with the dominant spectral features of the major sound-producing taxa on these reefs, fish and snapping shrimp, respectively. In the low-frequency band, the presence of humpback whales (December-May) was the major driver of sound level, whereas in the high-frequency band sound level closely tracked water temperature. On shorter timescales, the magnitude of the diel trend in sound production was greater than that of the lunar trend, but both varied in strength among reefs, which may reflect differences in the species assemblages present. Regression trees indicated that, at low frequencies, the relationship between species assemblages and acoustic parameters varied by season (i.e., the time that visual survey results were collected); however, at high frequencies, a given reef was generally most like itself over time. Thus, while long-term acoustic recordings can capture the substantial acoustic variability present in coral reef ecosystems, the broad-scale analyses presented here may not be sufficient to predict species assemblages from acoustic data alone. Indeed, a more granular approach might be needed.

\subsection{Introduction}

Coral reefs vary considerably in their species assemblages in both space and time (Parravicini et al., 2013; Williams et al., 2015). Accordingly, a large body of ecological literature has focused on trying to identify the drivers of this variability. Much effort has gone into characterizing links between biophysical attributes of coral reefs and fish species assem- 
blages. Parameters such as depth, substrate complexity (rugosity), live coral cover, and coral species richness appear to be important correlates with fish species richness and abundance (McCormick, 1994; Friedlander et al., 2003; Messmer et al., 2011; Komyakova et al., 2013).

Just as the biological composition of coral reefs changes over time, so too do the associated ambient soundscapes (Staaterman et al., 2014; Kaplan et al., 2015; Nedelec et al., 2015). For example, in an approximately year-long study of 2 Caribbean reefs, Staaterman et al. (2014) found that sound levels varied on diel and lunar scales. However, there was only a limited attempt to relate that variability to the species assemblages present. Kaplan et al. (2015) made a stronger effort to link the strength of diel trends in sound production to fish density and coral cover on 3 Caribbean reefs, but over a shorter (4-month) study. Nedelec et al. (2015) noted relationships between acoustic parameters that varied among 42 study reefs in French Polynesia and biophysical variables such as coral cover, fish density, sea state, etc., but used extremely brief recordings that likely overlooked the appreciable variation in sound level over longer timescales that has been demonstrated in other studies.

Collectively, these studies present initial characterizations of some of the putative drivers of the patterns of sound production, such as diel and lunar periodicities, water temperature, and the biota present. However, links between biophysical elements of a reef and the observed biological soundscape have not been robustly described. Furthermore, these drivers of biological sound production may vary in importance over time and among communities of sound-producing organisms. Thus, efforts to examine this variability must assess multiple study sites over long timescales to comprehensively elucidate these drivers and the magnitude of their contributions.

This growing body of work suggests that monitoring the sounds produced by the diverse array of soniferous coral reef organisms may be a cost-effective means of tracking reef community assemblages and their changes over time (Lammers et al., 2008). Acoustic observation could supplement or potentially obviate the need for frequent, traditional visual surveys by divers. However, to develop the capability to infer species assemblages and ecological dynamics from acoustic data (i.e., inverse prediction), it is first necessary to examine the relationship between biological sounds on reefs and fundamental ecological parameters that would be of interest to scientists and resource managers, such as fish species richness and abundance and live coral and algal cover, and, crucially, how that relationship may change 
over time.

In recent years, there has been considerable interest in applying acoustic biodiversity metrics developed for terrestrial ecosystems to marine soundscapes (e.g., Parks et al., 2014; Bertucci et al., 2016); however, there has been no compelling evidence to suggest that such metrics provide valuable information not available from more traditional measures like sound pressure level. For example, in a recent study, Bertucci et al. (2016) attempted to apply the Acoustic Complexity Index (ACI) to recordings of coral reefs. The authors claimed that higher ACI values found in recordings of coral reef Marine Protected Areas (MPAs) compared to non-MPAs would allow them to evaluate species richness within marine soundscapes. Confusingly, the authors also indicated that there were no significant differences between protected and unprotected areas in any visual survey parameter, thereby demonstrating that differences in ACI values among protected versus non-protected reefs are either spurious or not reflective of underlying species assemblages (Bertucci et al., 2016). At present, more traditional metrics such as sound pressure level and the variance in sound level over time are likely to be more robust and easier to comprehend than indices such as the ACI, which are expressed in arbitrary units.

In addition to biological sounds, anthropogenic noise such as engine noise from small boats can modify reef soundscapes in significant ways (e.g., Kaplan and Mooney, 2015). The extent of human activity can and does vary among reefs because of differing degrees of remoteness, protection (e.g., areas closed to vessels), and heterogeneous utilization rates. Recent work suggests that noise from small vessels may increase the predation risk for some reef fish (Simpson et al., 2016). Accordingly, these human-mediated elements could also play a role in driving biological sound production and species assemblages on coral reefs.

To parameterize the drivers of sound production on reefs across space, time, and ecological gradients such as live coral cover and fish density, long-term study of a range of geographically and ecologically disparate reefs is needed. This study attempted to fill that gap by examining the soundscapes and visually observable species assemblages at 7 reefs that varied in benthic cover, fish species assemblages, and degree of protection on the west and south sides of Maui, Hawaii, for an approximate 16-month period. 


\subsection{Methods}

\subsubsection{Site selection}

Reefs were selected for study on the west side of Maui, Hawaii, in September 2014. Reefs were chosen to be similar in depth but different in terms of benthic cover, fish species richness and individual abundance, structural complexity, geographic location, and degree of protection. These parameters were assessed in an ad hoc manner during the site selection period and confirmed ex post using visual surveys. Because of an instrument malfunction, 1 reef was ultimately excluded from the study, leaving 7 reefs for data analysis (Figure 3.1a; Table 3.1). Of these, 1 (Ahihi) was completely closed to vessel traffic, 2 were Marine Life Conservation Districts closed to some forms of fishing (Honolua and Molokini), and 1 was a Fishery Management Area closed to the fishing of herbivores (Kahekili).

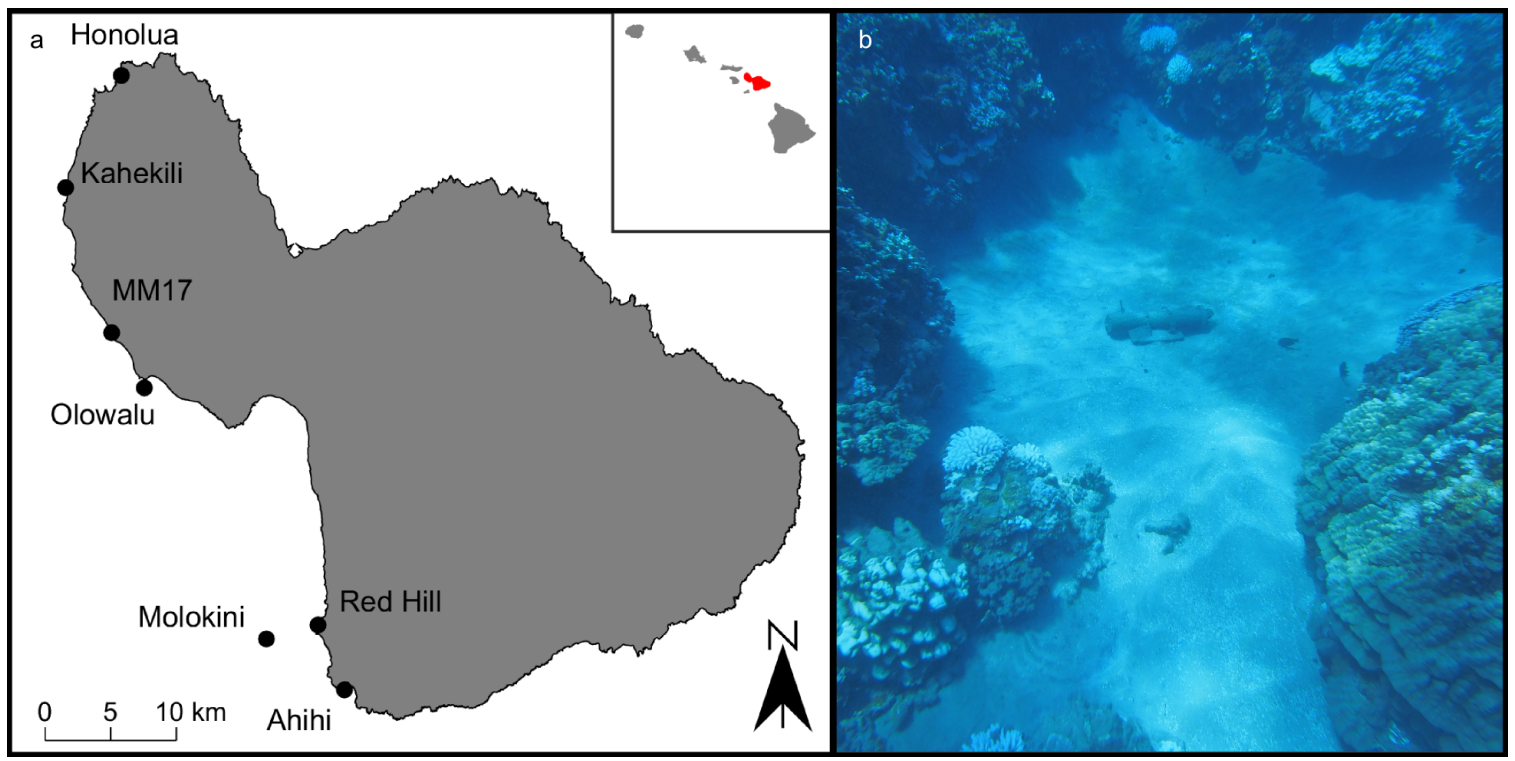

Figure 3.1: Map (a) depicting the location of the 7 study reefs in Maui, Hawaii and (b) an example of the EAR acoustic recorder deployed on reef Olowalu.

\subsubsection{Visual surveys}

Visual surveys were carried out at each study reef in September 2014, February/March 2015, October 2015, and January 2016. Data were collected by the same 2 divers for the duration of the study to ensure consistency among surveys. Four benthic transects per reef were conducted using a $10 \mathrm{~m}$ sinking lead line that followed the contours of the reef. Each 
transect started adjacent to the acoustic recorder moored at that reef and fanned out in a radial pattern. At each $10 \mathrm{~cm}$ increment, benthic cover was recorded as 1 of the following categories: live coral (identified to genus), macroalgae, turf algae, sand, bare rock, dead coral, bleached coral, and other invertebrates. Benthic cover data were later summarized across transects for each visual survey into the following categories: live coral, bleached coral, macroalgae, crustose coralline algae, turf algae, and "other" (e.g., bare rock, sand, dead coral, other invertebrates).

To quantify rugosity the straight-line distance of the lead line was measured with a fiberglass tape, and rugosity was calculated as the ratio of the length of the lead line to the length of the straight-line distance.

Four belt transect surveys for fish were carried out concurrently with the benthic surveys. These consisted of $30 \mathrm{~m}$ transects $(2.5 \mathrm{~m}$ on either side of the transect). Start points, adjacent to the acoustic recorder, were selected randomly. Each fish transect took approximately 10 min to complete. The surveyor first swam rapidly down the transect, recording larger mobile fishes transiting the line, mid-water species, and any conspicuous, rare, or uncommon species. They then turned around and returned down the transect, slowly and carefully recording all other fishes with a focus on cryptic species. Each observed fish was identified to species level and categorized by size (total length) in the following bins: A $(0-10 \mathrm{~cm}), \mathrm{B}(11-15$ $\mathrm{cm}), \mathrm{C}(16-20 \mathrm{~cm}), \mathrm{D}(21-30 \mathrm{~cm}), \mathrm{E}(31-40 \mathrm{~cm})$, and F $(>40 \mathrm{~cm})$. Fish survey data were pooled across transects and summarized by species and size classes. Whether a species was soniferous or not was assessed by comparing these results to a previously published list of soniferous Hawaiian reef fish (Tricas and Boyle, 2014).

Table 3.1: Summary of study reef location, degree of protection (Natural Area Reserve (NAR), Marine Life Conservation District (MLCD), and Fishery Management Area (FEA)), and physical attributes.

\begin{tabular}{l|l|l|l|l|l} 
Reef & Latitude & Longitude & Conservation/Protection status & Depth $(\mathrm{m})$ & Rugosity \\
\hline Ahihi & 20.59528 & -156.42462 & NAR & 9.1 & 1.36 \\
Honolua & 21.01577 & -156.64054 & MLCD & 6.2 & 1.43 \\
Kahekili & 20.93892 & -156.69470 & FMA & 11.2 & 1.25 \\
MM17 & 20.83076 & -156.64317 & N/A & 11.2 & 1.04 \\
Molokini & 20.63222 & -156.49602 & MLCD & 14.6 & 1.18 \\
Olowalu & 20.80202 & -156.61855 & N/A & 10.1 & 1.24 \\
Red Hill & 20.63982 & -156.45059 & N/A & 7.1 & 1.24
\end{tabular}




\subsubsection{Acoustic data}

Acoustic data were collected at each reef using Ecological Acoustic Recorders (EARS, Lammers et al. 2008) equipped with SQ26-01 hydrophones (Sensor Technology Ltd., Collingwood, ON, Canada) with sensitivities of approximately $-193.5 \mathrm{dBV}$ re $1 \mu \mathrm{Pa}$ and configured with $47.5 \mathrm{~dB}$ of gain. Recordings were collected at sample rates of $50 \mathrm{kHz}(25 \mathrm{kHz}$ at Molokini) on a $10 \%$ duty cycle $(30 \mathrm{~s} / 300 \mathrm{~s})$. For all deployments, EARs were affixed to concrete blocks using hose clamps and cable ties and placed in sand patches adjacent to or within a reef (Figure 3.1b). All EARs except at Molokini were deployed in September 2014, refurbished in February/March 2015 and July 2015, and recovered in January 2016. The Molokini EAR was deployed and refurbished on a different schedule (November 2013, June 2014, October 2014, February 2015, October 2015, October 2016).

Analyses were carried out in MATLAB 9.1 (MathWorks, Natick, MA). Any 30 s sound file that contained normalized voltage readings of \pm 1 , indicating the presence of clipping, were automatically excluded (Table 3.2). However, because of rounding in and timing of the analog-to-digital converter it is possible that clipping could occur at values lower than \pm 1. All remaining sound files were individually analyzed as follows. Files were corrected for hydrophone sensitivity and resampled to $44 \mathrm{kHz}$ for improved computational efficiency and so as to retain frequencies of interest (except for recordings from Molokini, which were not resampled because of the lower sample rate). Root-mean-square (RMS) sound pressure level $(\mathrm{dB}$ re $1 \mu \mathrm{Pa})$ was calculated in 2 frequency bands - low $(50-1200 \mathrm{~Hz})$ and high $(1800-$ $20500 \mathrm{~Hz}$; 2000-12000 Hz for Molokini) - using four-pole Butterworth bandpass filters. While a narrower bandwidth at high frequencies was used for Molokini, this choice did not affect results because no explicit comparisons of sound levels among reefs were made. These bands were chosen to correspond with the published frequency ranges of fish and snapping shrimp calls, respectively (Tricas and Boyle, 2014; Au and Banks, 1998). Power spectra were produced for all files across the full band $(50-20500 \mathrm{~Hz} ; 50-12000 \mathrm{~Hz}$ for Molokini because of the lower sample rate) using Welch's method (Welch, 1967) with a Fast Fourier Transform (FFT) size of 880 points (500 for Molokini), 75\% overlap and a Hann window. Octave band levels $(\mathrm{dB}$ re $1 \mu \mathrm{Pa})$ were calculated for each file at centroid frequencies of 125, 250, 500, 1000, and $2000 \mathrm{~Hz}$ using digital filters (Radford et al., 2014). All calculations except for that of the power spectrum were also made on every $100 \mathrm{~ms}$ segment of each file to assess 
within-recording variability over shorter time scales.

Table 3.2: Number of $30 \mathrm{~s}$ recordings collected at each reef over the study period and the number and percentage retained after exclusion based on clipping or presence of anthropogenic noise.

\begin{tabular}{l|l|l|l} 
Reef & $\begin{array}{l}\text { Total number of } \\
\text { recordings made }\end{array}$ & $\begin{array}{l}\text { Number of recordings after } \\
\text { exclusion of clipped recordings } \\
\text { and recordings with anthropogenic } \\
\text { noise }\end{array}$ & $\begin{array}{l}\text { Percentage of } \\
\text { recordings analyzed }\end{array}$ \\
\hline Ahihi & 110219 & 104264 & 94.6 \\
Honolua & 116651 & 110598 & 94.8 \\
Kahekili & 108264 & 104605 & 96.6 \\
MM17 & 119845 & 91075 & 76 \\
Molokini & 178672 & 74190 & 41.5 \\
Olowalu & 94357 & 67753 & 71.8 \\
Red Hill & 127444 & 102043 & 80.1
\end{tabular}

Because there can be appreciable amplitude and frequency variation within sound files, SPL alone may not be adequate to characterize the levels of biological sound production in each recording. Indeed, quantification of the magnitude of the within-recording amplitude and spectral variability should be expressed in physical units. Accordingly, the median absolute deviation (i.e., the median absolute deviation from the median level; MAD) was computed as follows. First, MAD was computed individually for octave bands at 125, 250, 500, and $1000 \mathrm{~Hz}$ in $100 \mathrm{~ms}$ windows and then averaged (on the linear scale in Pascals) across those octave bands to generate a single low-frequency value for each recording. A similar approach was used for the octave band at $2000 \mathrm{~Hz}$ to capture the complexity at high frequencies. This metric describes within-recording variability over time and frequency in $\mathrm{dB}$ values, and is an accurate and simple representation of the extent to which there are relatively high-amplitude transients followed by comparatively low-amplitude background noise.

To ensure that these analyses focused on sounds of biological origin, vessel and other extrinsic anthropogenic noise was identified and excised. This was done individually for each reef by visually identifying and aurally confirming such sounds in long-term spectral average plots produced in Triton version 1.91 (Scripps Whale Acoustics Lab, San Diego, CA).

The presence of humpback whales (Megaptera novaengliae) during the winter months (approximately December-May) represented an undesired biological sound source, in particular when making among-reef comparisons of low-frequency sound, where humpback whale 
song overlaps with and can significantly mask lower amplitude fish calls. Thus, low-frequency sound data were not considered between 1 December-30 April except in visualizations of daily average levels over the total deployment period.

To calculate the strength of the diel increases in sound production at the crepuscular times, the difference between the median sound levels at dawn and day and between dusk and day were calculated. This was done so that comparisons among reefs could be made irrespective of reef-specific background noise levels. Dawn was defined as 1 hour before to 15 minutes after sunrise, dusk was defined as 15 minutes before to 1 hour after sunset, and day was defined as 15 minutes after sunrise to 15 minutes before sunset.

Comparisons between diel and lunar periodicity were made by constructing periodograms of the SPL time series in both frequency bands. Linear interpolation to fill in missing data was necessary to ensure a constant sampling rate of 1 recording/5 min or 288 samples/day.

\subsubsection{Environmental parameters}

Temperature data loggers (HOBO pendant models UA-001-64 and UA-002-64, Onset Computer Corporation, Bourne, MA), sampling every 10 minutes, were deployed alongside EARs at all reefs for the duration of the study, except for Molokini, where temperature data were only collected from July 2015 until January 2016. Sunrise and sunset were computed for each day of the deployment in MATLAB using the coordinates of each reef with the suncycle tool from the AIR SEA TOOLBOX (v. 2.0). Wind speeds were gathered from a nearby NOAA National Ocean Service weather buoy $\left(20.895^{\circ} \mathrm{N}\right.$, $\left.156.469^{\circ} \mathrm{W}\right)$. Lunar illumination data were obtained from the US Naval Observatory website (http://aa.usno.navy.mil/data/docs/MoonFraction.php).

\subsubsection{Statistical analysis}

Correlations between wind speed and SPL were computed using hourly averages for each variable. Correlations between temperature and SPL were computed using daily averages for each variable. Visual survey data were correlated with acoustic data coinciding $( \pm 30$ days) with the survey dates to avoid the possibility that the biological components of the reef might change in between surveys. Accordingly, high-frequency correlations were made at each of the 4 visual survey periods whereas low-frequency correlations were only made for visual surveys conducted in September 2014 and October 2015, to avoid including any 
acoustic data that contained humpback whale song.

Regression trees were used to evaluate the biophysical drivers of biological sound production and their relative importance (De'ath and Fabricius, 2000). Predictor variables were number of soniferous fish less than $15 \mathrm{~cm}$ total length, number of soniferous fish greater than $15 \mathrm{~cm}$ total length, number of soniferous fish species, and percent cover of coral, bleached coral, macroalgae, turf algae, crustose coralline algae, and "other". The response variable was computed using principal component analysis (PCA) and was equal to the first principal component of the following variables: the strength of the diel trend and MAD values for dawn and dusk for both the new and full moon within the relevant sampling month (i.e., overlapping with the visual survey dates). These variables were normalized prior to PCA. Accordingly, there were 8 predictor variables reduced using PCA for each frequency band. Samples (i.e., reefs at a given sampling time) that were missing any of the above response variables were excluded to ensure fair comparisons among reefs.

Regression trees were fitted in MATLAB using fitrtree, and overfitting was forced such that each observation was represented by a unique leaf node. Predictor variable importance was assessed using the MATLAB function predictorimportance, which computes the sum of changes in mean squared error (MSE) due to splits on every predictor and then divides that sum by the number of branch nodes.

\subsection{Results}

Benthic cover varied among and within the study reefs (Figure 3.2a). Live coral cover was generally highest at Molokini and Olowalu and lowest (i.e., zero) at MM17, a sandy non-reef control site. Honolua had the highest proportion of turf algae and Ahihi had the highest crustose coralline algae cover. Within-reef cover was relatively consistent over time except during the October 2015 survey, when an appreciable proportion of live coral was bleached at every reef except MM17. Reefs that contained the highest amount of live coral, such as Molokini and Olowalu, also had the greatest proportion of bleaching. By January 2016, the majority of this bleaching had diminished and recovery was observable at every bleached reef, although some, such as Red Hill, suffered mortality.

Corals from the genus Porites dominated live coral cover at Ahihi, Kahekili, Olowalu, and Red Hill, whereas corals from the genus Montipora were dominant at Molokini. At 
Honolua, live cover was more evenly split between Porites and Montipora corals. Other observed genera included Pocillopora, Pavona, and Fungia.

Fish survey results were less consistent, with both individual abundance and observed number of species following different trends at each reef (Figure 3.2b-c). For example, both individual abundance and species richness appeared to decrease over time at Ahihi while staying relatively flat at Kahekili and increasing and then decreasing at Red Hill. Nevertheless, there were some consistent patterns. MM17 always had the lowest species richness and individual abundance and Kahekili and Red Hill consistently demonstrated the highest individual abundance, whereas the observed number of species appeared to reach an asymptote at Kahekili, Molokini, and Red Hill.
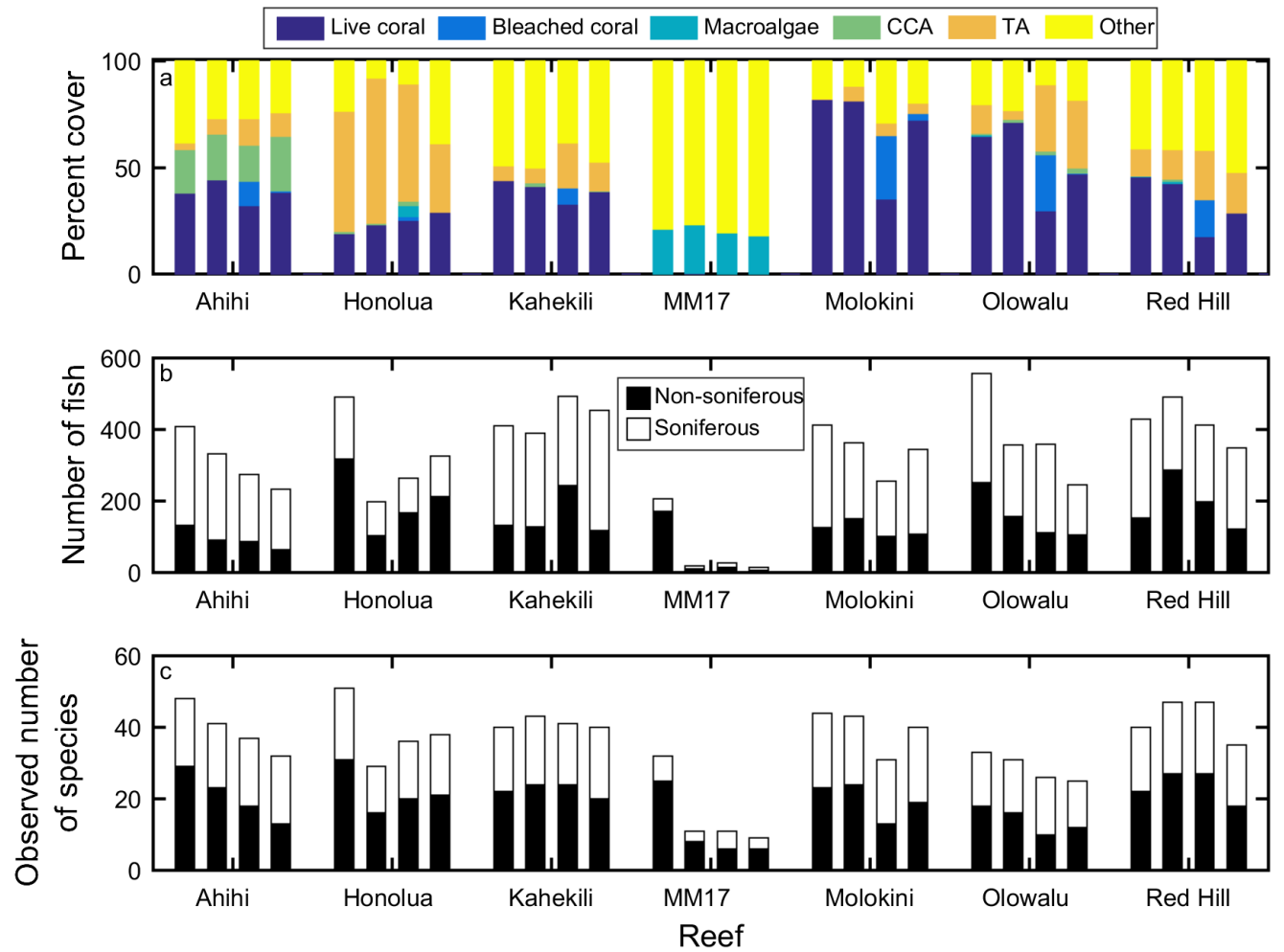

Figure 3.2: Visual survey results by reef (alphabetical order) and survey period (bars in order from September 2014, February/March 2015, October 2015, January 2016). Benthic cover (a) varied among and within reefs, in particular during the bleaching event of October 2015, but bleached coral largely recovered by January 2016. The (b) abundance of both soniferous and non-soniferous fish also varied among reefs and over time but was consistently lowest at MM17 and highest at Kahekili and Red Hill. Similarly, (c) fish species richness varied but did not appear to vary considerably among reefs with the exception of MM17, where the fewest species were represented. Data on sound production were obtained from Tricas and Boyle (2014). 
The proportion of soniferous fish individuals and species varied among surveys and reefs but in general was approximately half of the total. For fish up to $15 \mathrm{~cm}$ total length (i.e., small fish), the most commonly observed soniferous species was the goldring bristletooth (Ctenochaetus strigosus, Acanthuridae). At MM17, the most common small soniferous species was the Hawaiian dascyllus (Dascyllus albisella, Pomacentridae) and at Molokini it was the blacklip butterflyfish (Chaetodon kleinii, Chaetodontidae). There was more variation among reefs, and within reefs among surveys, in terms of the most abundant soniferous fish species larger than $15 \mathrm{~cm}$. Representative families included Acanthuridae, Balistidae, Chaetodontidae, Holocentridae, Labridae, Monacanthidae, Mullidae, Pomacentridae, Serranidae, and Zanclidae.

Small soniferous fish abundance and soniferous fish species richness appeared to correlate positively but noisily with live (unbleached) coral cover; however, no such relationship was obvious for large soniferous fish abundance (Figure 3.3).

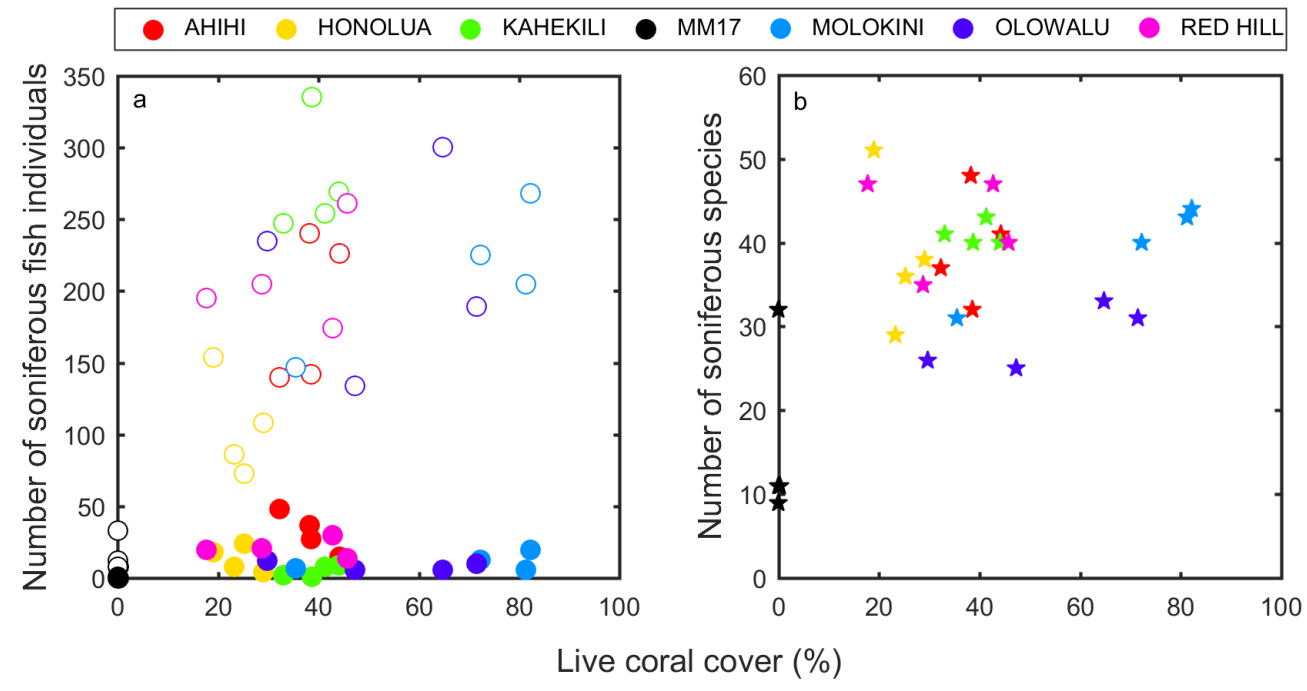

Figure 3.3: Relationship between (a) number of soniferous fish individuals (small - open circles; large - filled circles) and live (unbleached) coral cover and (b) number of soniferous fish species and live coral cover. Results from all 4 visual survey periods are included here. These data indicate a positive but noisy correlation between small soniferous fish abundance and live coral cover, and soniferous fish species richness and live coral cover.

Low-frequency SPL followed a strongly seasonal pattern at all sites except Ahihi, with daily average SPL in winter elevated by over $20 \mathrm{~dB}$ as a result of the presence of singing humpback whales (Figure 3.4). High-frequency SPL did not demonstrate such strong seasonality, and levels were much flatter over the course of the year. High-frequency levels 
appeared elevated at Ahihi by 2-3 dB after instrument redeployment in July 2015 compared to other deployment periods. No such elevation was apparent in low-frequency levels, which suggests that this shift could be a result of a change to instrument orientation during the redeployment process.
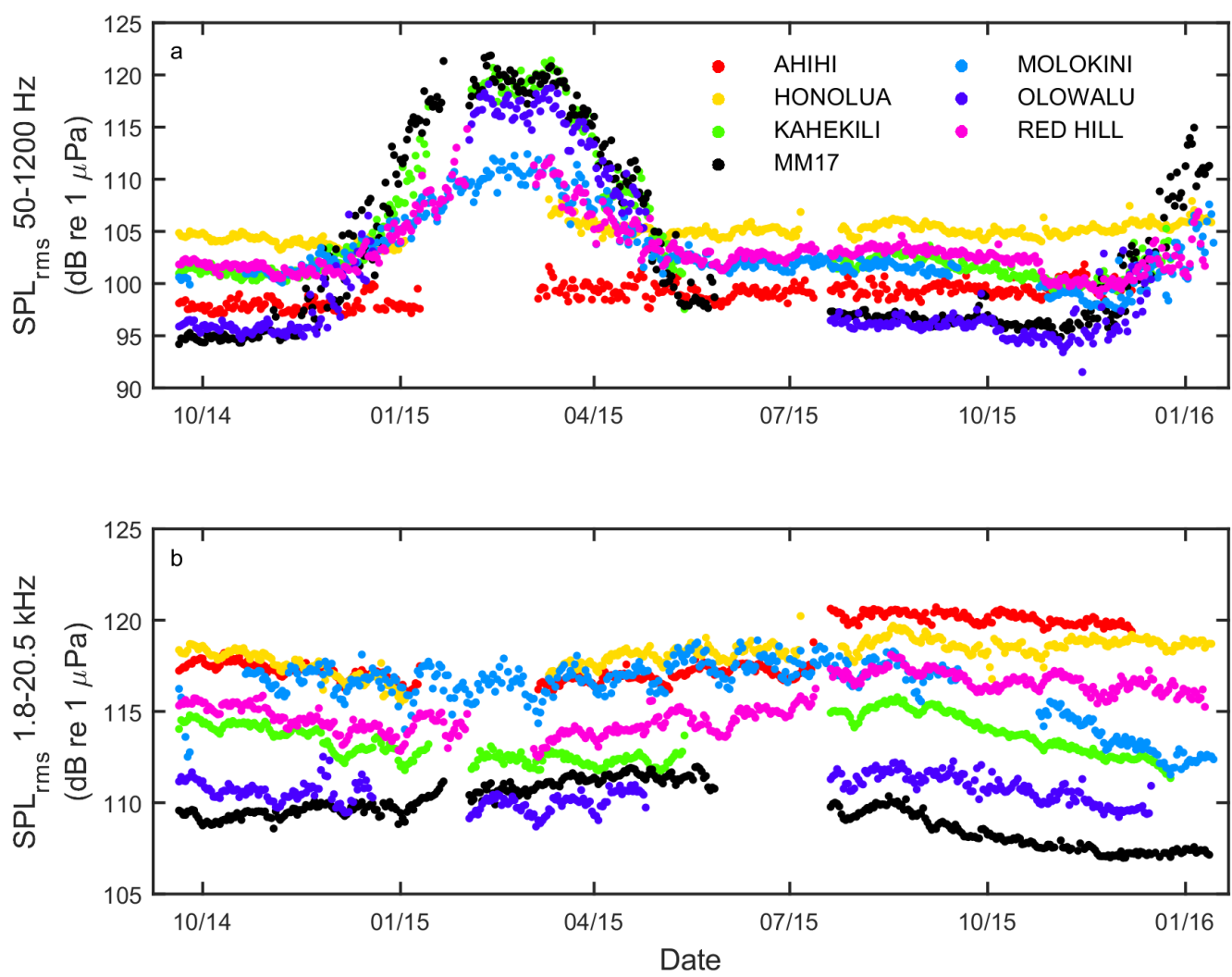

Figure 3.4: Daily average sound pressure level (SPL) in the (a) low- and (b) high-frequency band for the duration of the study at each reef. Peaks in sound level in the winter are evident in the low-frequency band as a result of the presence of singing humpback whales. Sound level in the high-frequency band appears to oscillate to a smaller degree and over shorter timescales.

There did not appear to be any correlation between wind speed and high-frequency SPL (Figure 3.S1) or between temperature and low-frequency SPL (Figure 3.5a, Figure 3.S2) at any reef; however, correlations between temperature and high-frequency SPL (Figure 3.5b, Figure 3.S3) were positive and relatively strong at every reef except MM17, the sandy control site, where the correlation was negative.

Sound pressure level at Kahekili, representative of trends at other reefs, was generally highest during the new moon at dawn compared to other times of day and moon phases 


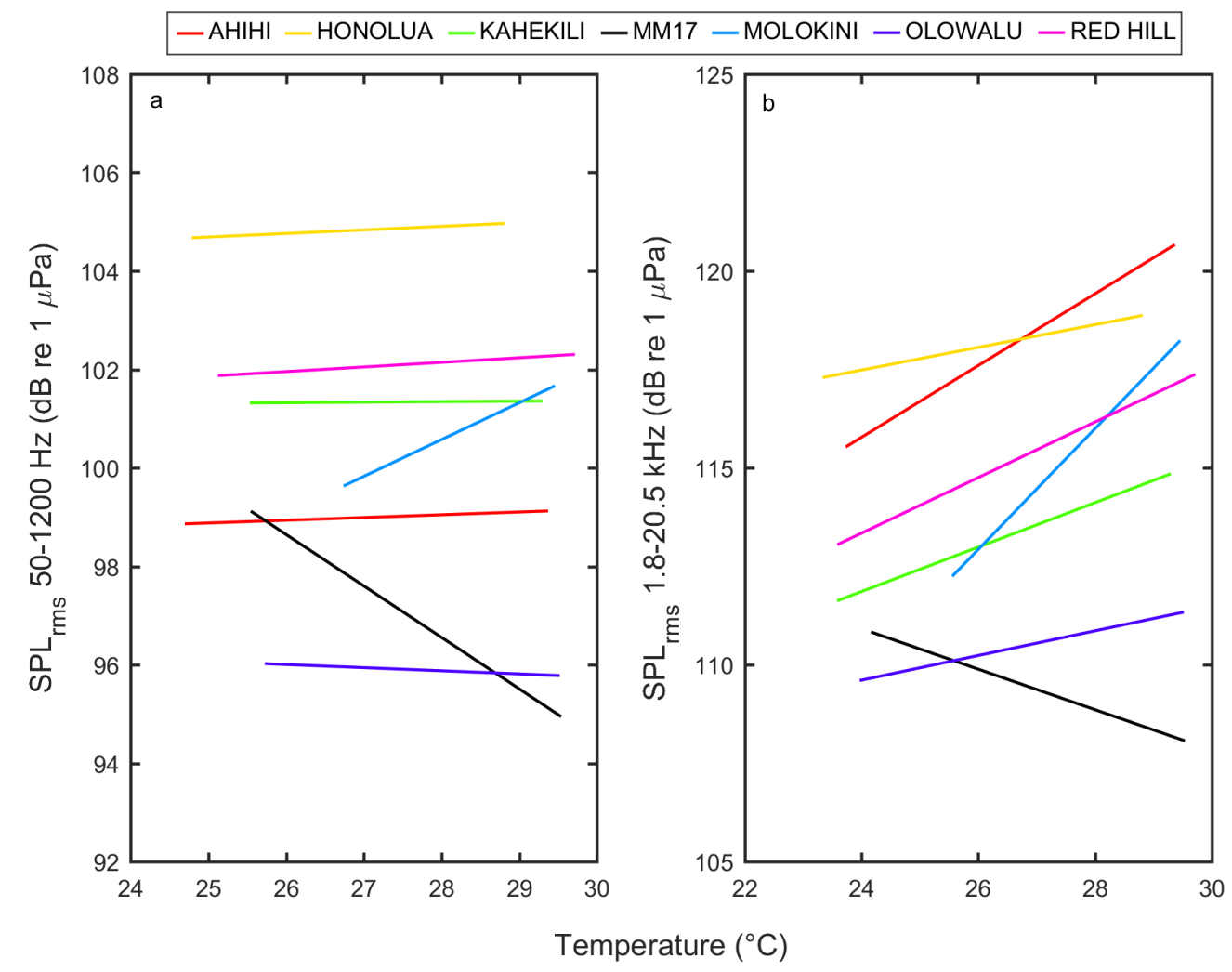

Figure 3.5: Linear regression lines of daily average water temperature and SPL at (a) low and (b) high frequencies across the study reefs. There was no general relationship apparent at low frequencies; conversely, at high frequencies positive correlations between temperature and SPL were seen at all reefs except for MM17, the sandy control site. Equations of the lines and evaluations of fit are included in Figures 3.S2-3.

(Figure 3.6a), except at MM17 where there was no obvious diel or lunar trend in sound level (Figure 3.6). At high frequencies, levels were typically highest at the full moon at dusk (Figure 3.6b).

Characteristic peaks in SPL at dawn and dusk were evident in both frequency bands (Figure 3.7). After excluding times when humpback whales were present, the maximum SPL on a given day at low frequencies was often located around the crepuscular periods. At high frequencies, peak levels were almost always found before dawn or after dusk, reflecting the strong diel nature of snapping shrimp activities. At some reefs, such as Honolua, lowfrequency SPL appeared to stay somewhat elevated during the night between the dusk and dawn peaks, whereas at most other reefs the night levels were as low as the day levels. This divergence at Honolua was likely a result of shrimp snaps extending into lower frequencies than at other reefs (Figure 3.8). 

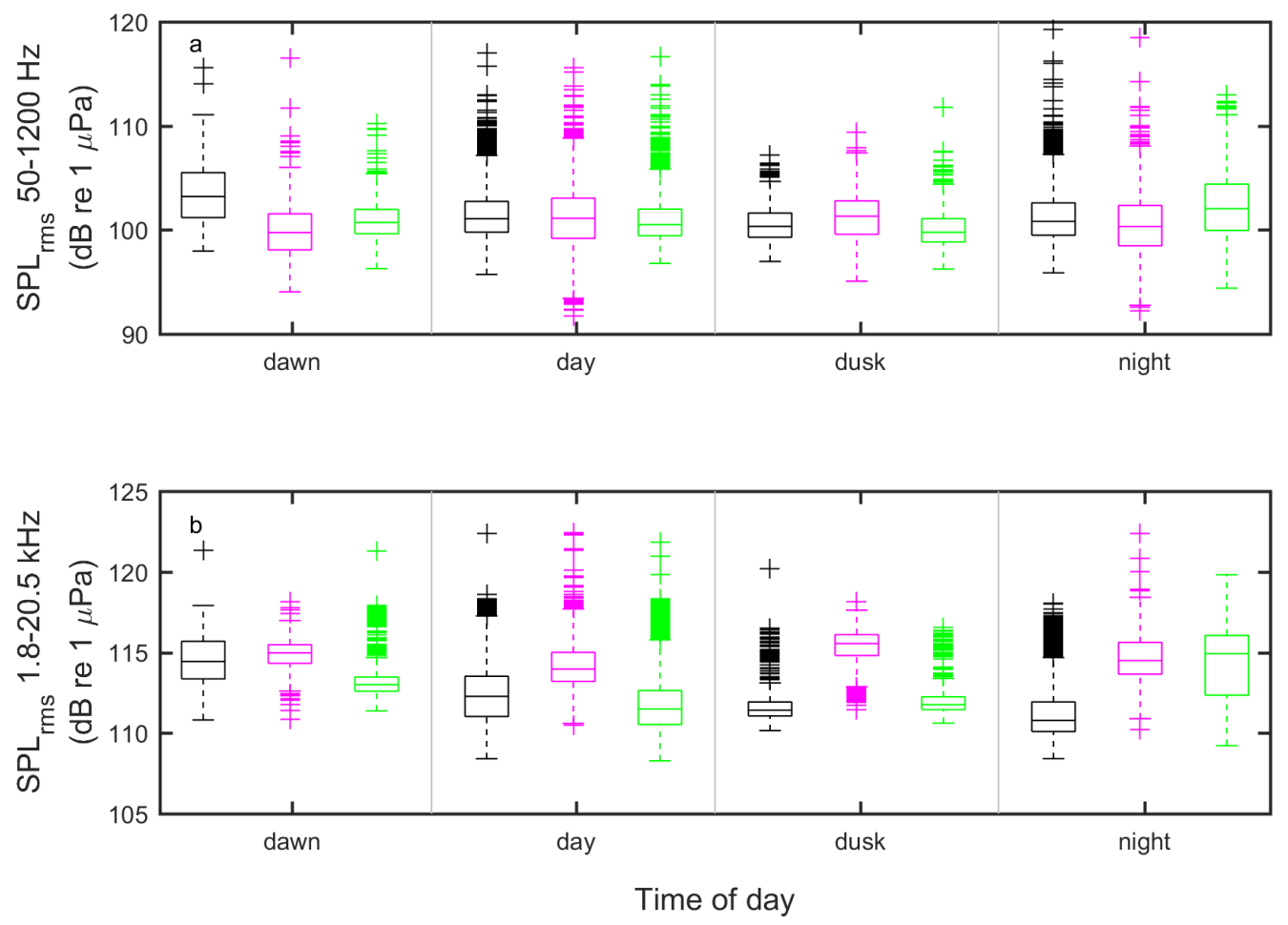

Figure 3.6: Boxplots presenting (a) low- and (b) high-frequency SPL at Kahekili during the new (black) and full (purple) moon and first/last quarter (green) at 4 times of day. At low frequencies, sound levels were typically highest during the new moon at dawn compared to all other times whereas at high frequencies dusk full-moon levels tended to be highest. Results were similar for other reefs, except MM17 where lunar and diel variability was limited in both frequency bands.

Trends in median absolute deviations (MAD), which reflect both spectral and amplitude variability within each 30 s sound file, followed a similar diel pattern to SPL on some but not all reefs (Figure 3.9). Honolua in particular demonstrated much greater crepuscular MAD values in the low-frequency band. Conversely, at high frequencies, Honolua levels were consistently high throughout the day and did not demonstrate diel periodicity. This is unlike other reefs, which in general demonstrated elevated levels between dusk and dawn. Elevated MAD levels at low frequencies at Honolua were likely driven in part by shrimp snaps that appeared to extend into considerably lower frequencies than is typical (Figure $3.10)$.

The magnitude of the diel trend appeared to be much greater than that of the lunar trend. Indeed, at Kahekili, the reef with the strongest lunar trend, diel periodicity was nearly 4 times stronger than lunar periodicity at both low and high frequencies (Figure 

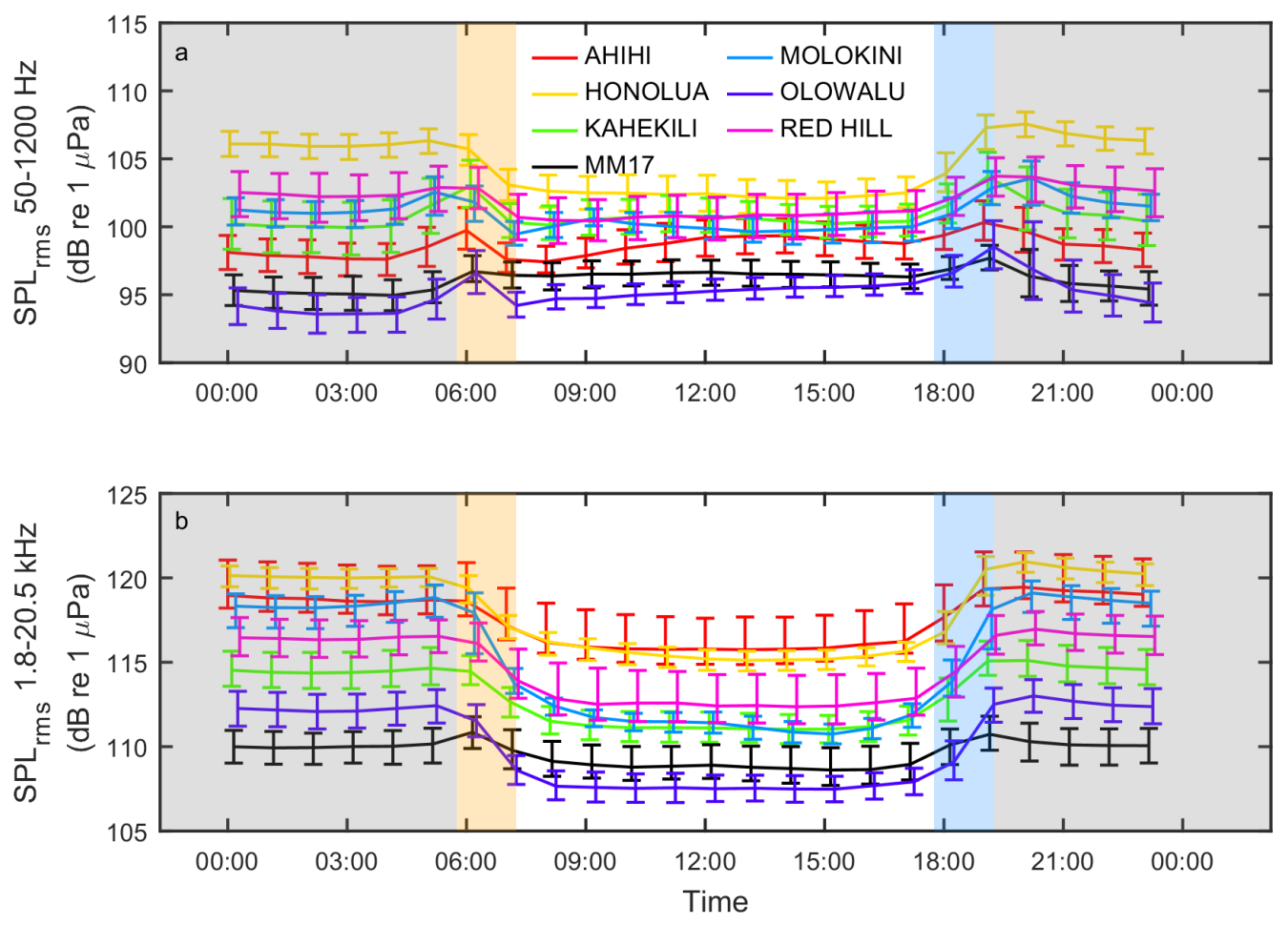

Figure 3.7: Median SPL (25-75 percentiles) at (a) low- and (b) high-frequency for each reef by hour of the day. There were elevated levels at every reef at dawn (orange shaded) and dusk (blue shaded). However, this pattern varied somewhat among reefs. For example, at Honolua, low-frequency levels remained elevated throughout the night (grey shaded), whereas at other reefs levels only were elevated at the crepuscular periods.

3.11). The excess strength in the diel trend was even greater for other reefs.

The relationship between biological attributes of a reef determined from the visual surveys and acoustic metrics varied between the 2 frequency bands of interest (Figure 3.1213). At low frequencies, reefs clustered based on survey period. Percent bleached cover, which was approximately zero in September 2014, was higher on nearly every reef in October 2015 and accordingly appears to have been an important factor in structuring the tree (Figure 3.12), although the magnitude of the difference in acoustic variables between these 2 sampling periods appears to be small. The most important structuring variables for the low-frequency acoustic attributes were the number of soniferous fish species and percent turf algae cover (Figure $3.14 \mathrm{a})$.

At high frequencies, 3 main categories emerged: Honolua, MM17 and Kahekili, and all others (Figure 3.13). Reefs did not appear to cluster based on sampling period, in contrast 

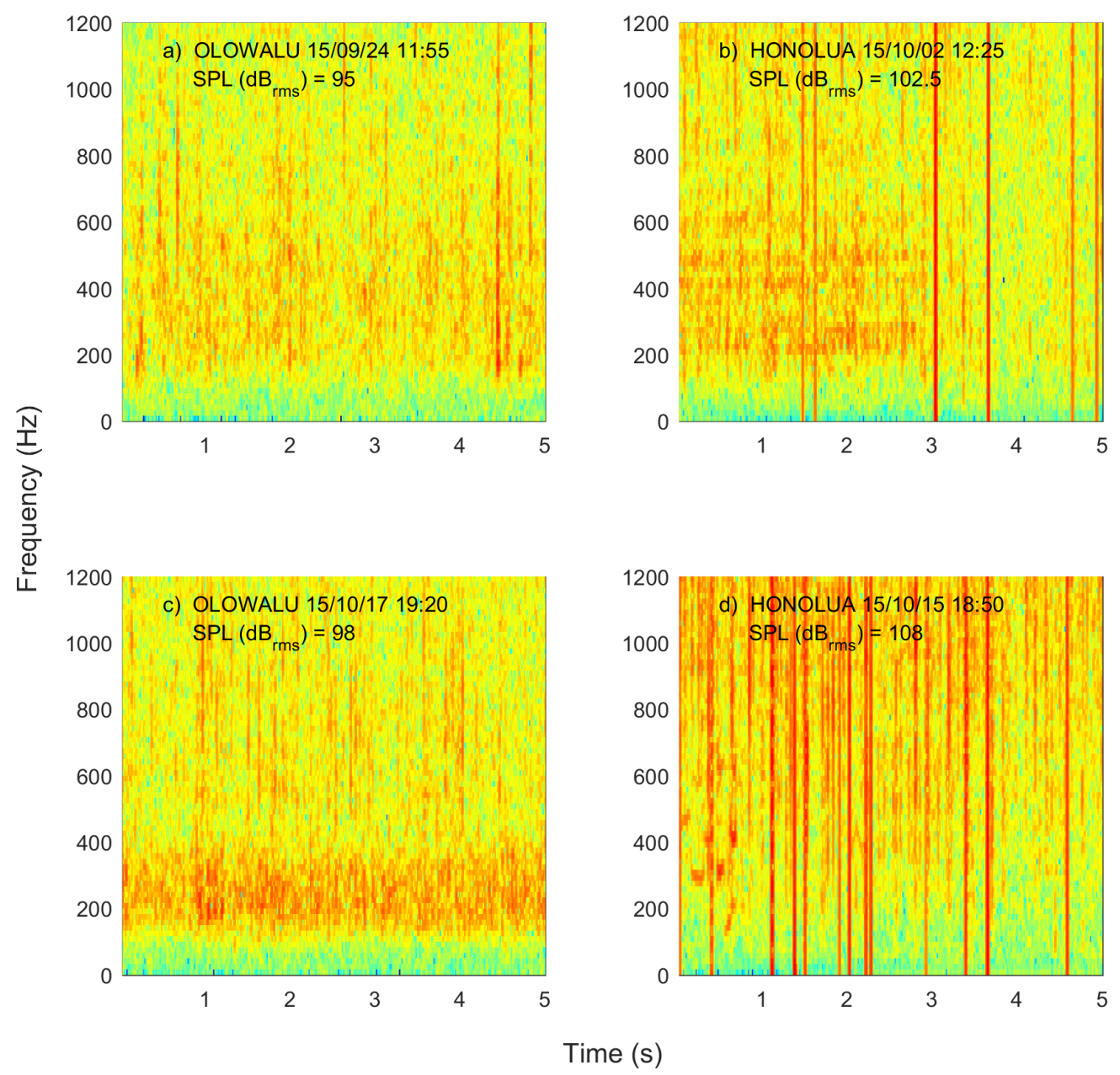

Figure 3.8: Spectrograms depicting $5 \mathrm{~s}$ of sound from around noon and around $8 \mathrm{pm}$ at $(\mathrm{a}, \mathrm{c})$ Olowalu and (b,d) Honolua at low frequencies and corresponding SPL values $(50-1200 \mathrm{~Hz}$, $\mathrm{dB}$ re $1 \mathrm{\mu Pa}$ ). These values were around the median values from the entire deployment period for these reefs at these times. SPL tended to increase at low frequencies at both reefs from midday to dusk. At Olowalu, the dusk increase appeared to be driven by elevated fish calling activity but was relatively limited, whereas at Honolua the increase was more likely a result of high-amplitude shrimp snaps extending into the lower frequencies (potentially indicative of clipping). Spectrogram settings: FFT size $=3000,75 \%$ overlap, Hann window, dynamic range $=30-90 \mathrm{~dB}$ re $1 \mathrm{\mu Pa}^{2} \mathrm{~Hz}^{-1}$.

to the low-frequency results. The most important predictor variable at high frequencies was turf algae (Figure 3.14b). 

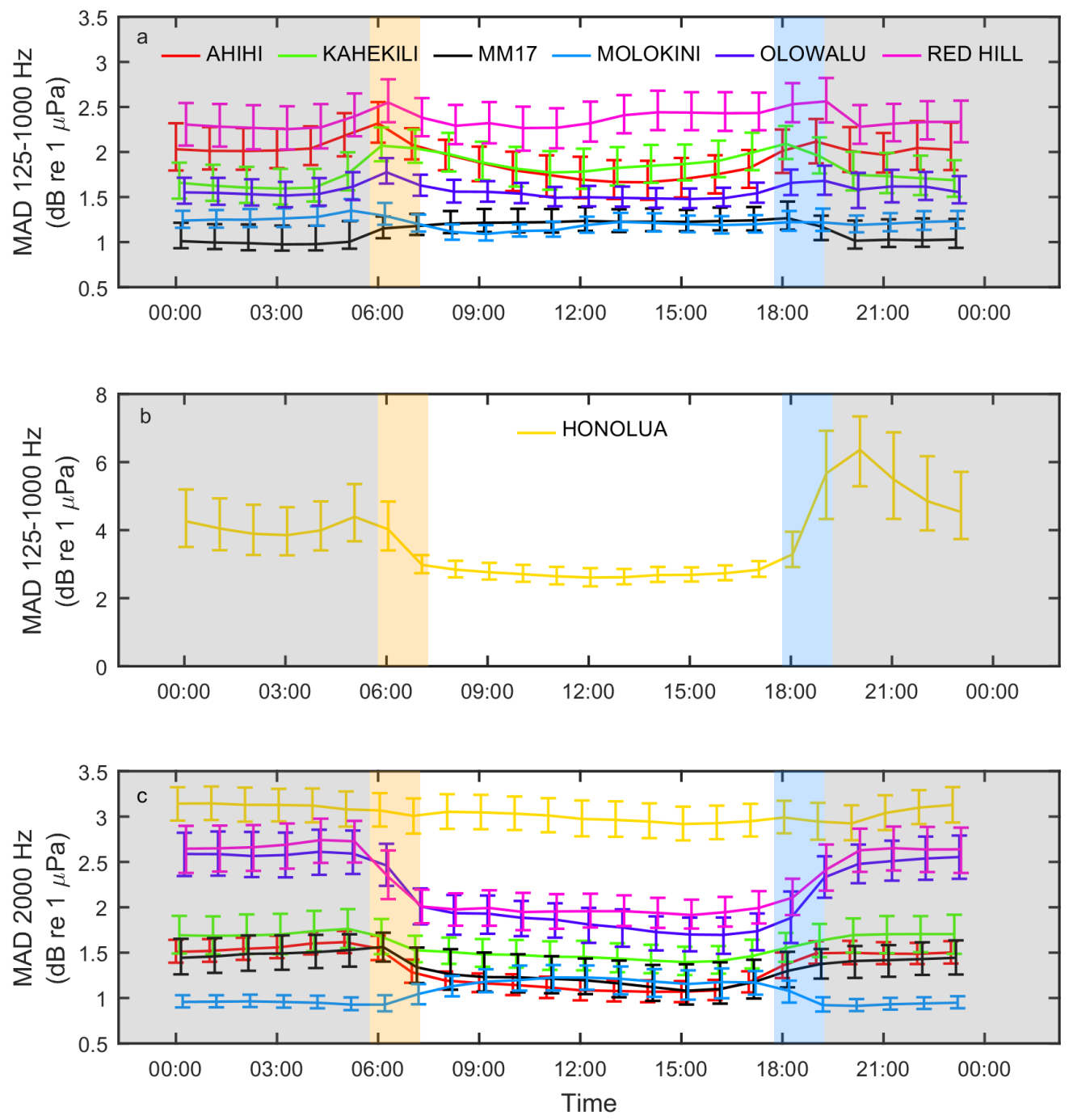

Figure 3.9: Median MAD values at $(\mathrm{a}-\mathrm{b})$ low and (c) high frequencies for each reef by hour of the day. While at (a) low frequencies many reefs demonstrated a similar pattern to amplitude periodicity with small peaks at dawn and dusk, (b) Honolua had much higher overall MAD levels, potentially as a result of clipping, and considerable elevations at the crepuscular periods (and therefore is plotted separately). Conversely, at (c) high frequencies Honolua was relatively flat over the diel period whereas other reefs were periodic with elevated levels between dawn and dusk. Molokini appeared to demonstrate the opposite pattern, with higher MAD levels during the day.

\subsection{Discussion}

The goal of this study was to better understand the drivers of biological sound production on coral reefs and the extent to which acoustic records reflect fundamental ecological parameters 

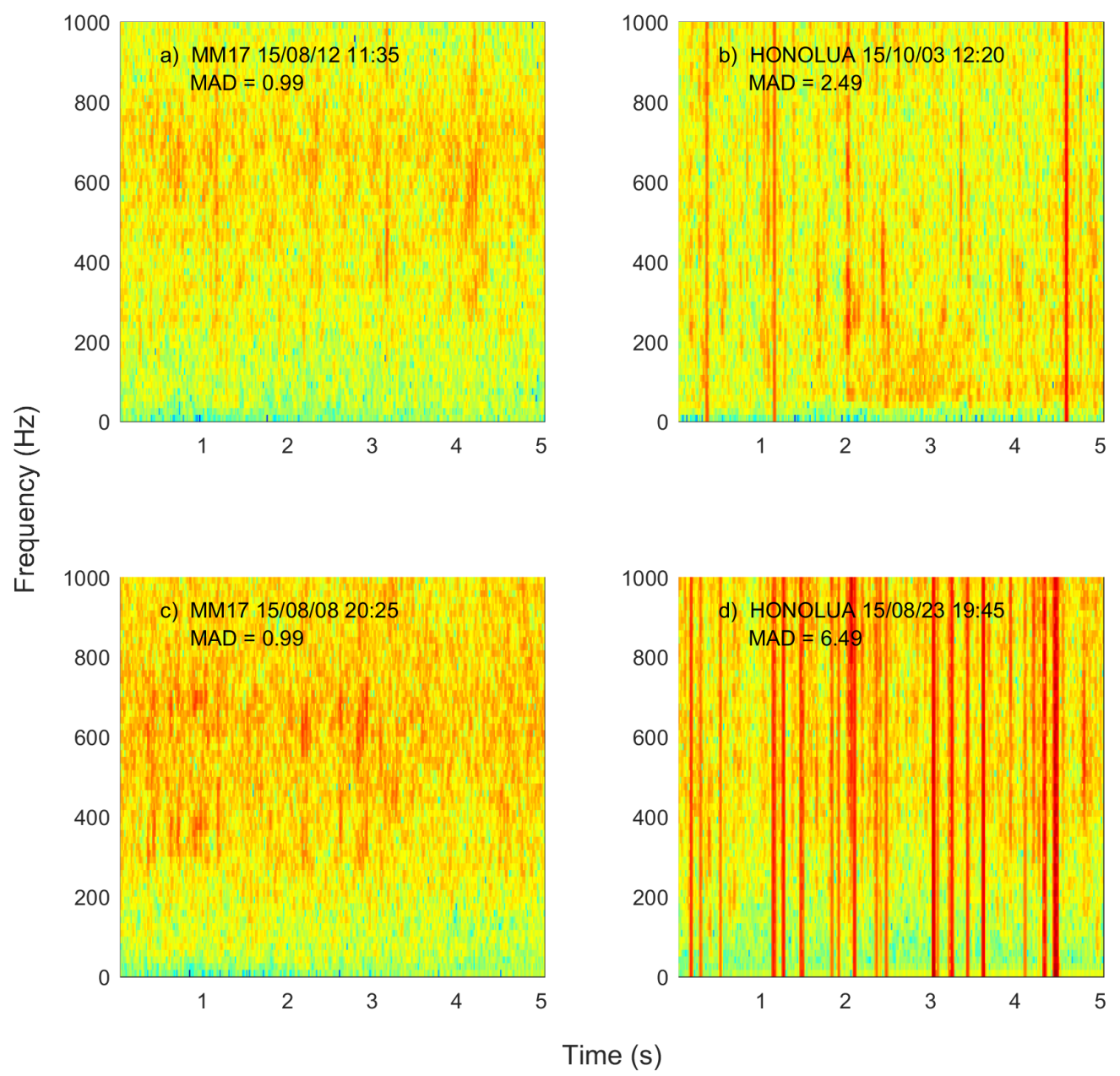

Figure 3.10: Spectrograms depicting $5 \mathrm{~s}$ of sound from around noon and around 8pm at $(\mathrm{a}, \mathrm{c}) \mathrm{MM} 17$ and $(\mathrm{b}, \mathrm{d})$ Honolua at low frequencies and corresponding MAD values. These values were around the median values from the entire deployment period for these reefs at these times. MAD was particularly low at MM17 at all times of day. At Honolua, MAD was higher in general and particular around 8pm. These elevated levels appear to be driven by shrimp snaps that extend into the low-frequency band (potentially indicative of clipping). Spectrogram settings: FFT size $=3000,75 \%$ overlap, Hann window, dynamic range $=30-90$ $\mathrm{dB}$ re $1 \mathrm{\mu Pa}^{2} \mathrm{~Hz}^{-1}$.

such as live coral cover and reef fish biodiversity. Results indicate that reefs that were similar in their visual survey attributes clustered together acoustically in the CART regression trees. At the same time, reefs that were very different visually were far apart in the trees, for example, MM17 and Honolua. However, there was insufficient spread among reefs to be confident that acoustic data alone would be adequate to infer species assemblages on 


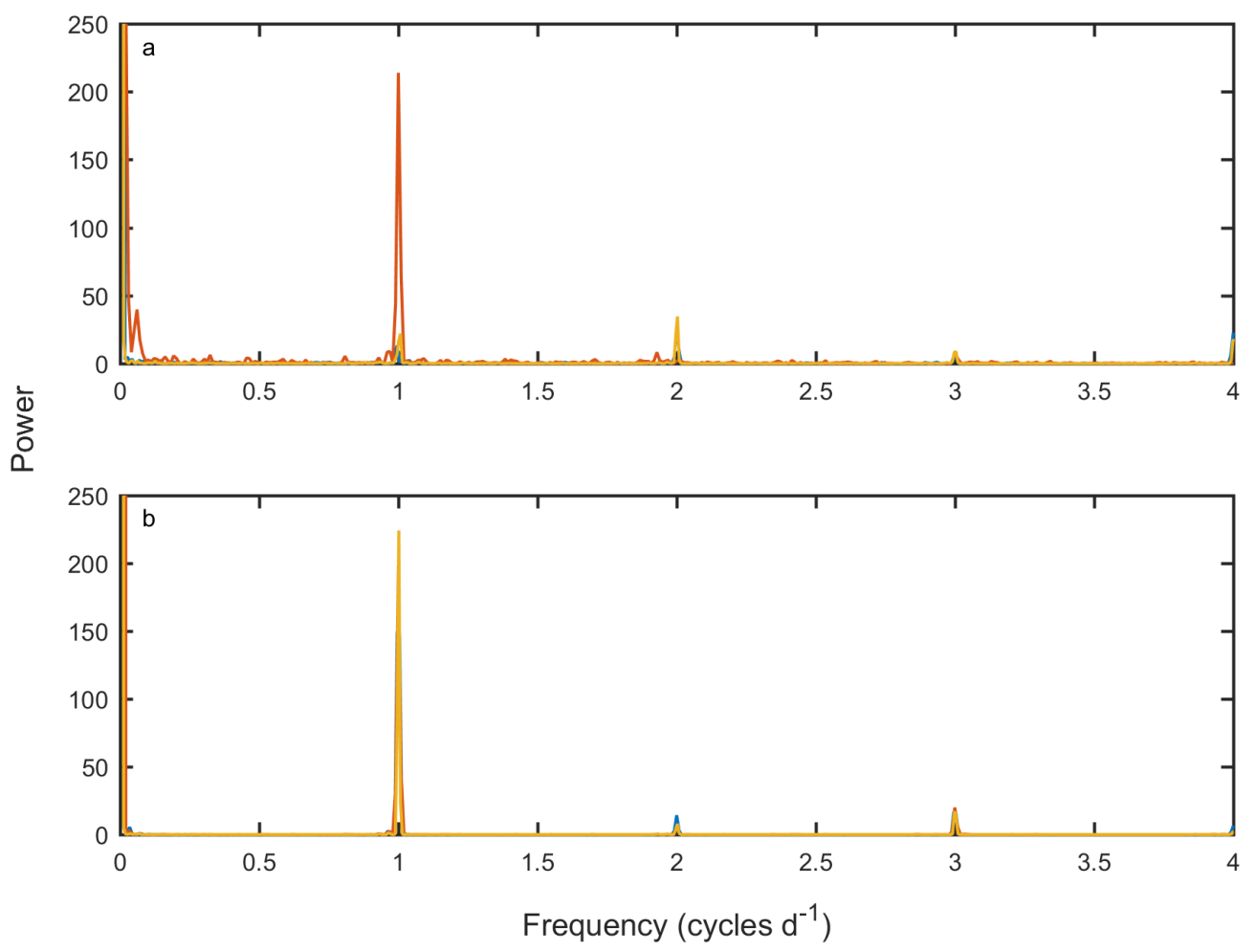

Figure 3.11: Magnitude of periodicity in SPL at (a) low and (b) high frequencies for Kahekili. Diel periodicity was the strongest in both frequency bands, with clear peaks at 1 cycle per day and, at low frequencies, an additional peak at 2 cycles per day. Lunar periodicity was evident at low frequencies (peak near 0) but not high frequencies. Colors represent individual deployment periods.

reefs at this time. Thus, the prospect of performing inverse predictions based on broadscale acoustic results remains elusive. A more granular approach, such as identifying the occurrence patterns of individual sound types previously identified to the species level and correlating those with visual survey data, might be more effective.

The CART regression analysis indicated a divergence between frequency bands in terms of change over time. At low frequencies, the presence of coral bleaching in October 2015 appeared to drive the clustering of reefs in terms of their acoustic response variables; indeed, percent bleached coral cover partitioned the reefs by survey date (October 2014 and October 2015) for all reefs except MM17 (where bleached coral cover was $0 \%$ in both surveys), which indicates that perhaps the presence of bleached coral had similar effects across reefs. Conversely, at high frequencies, reefs tended to cluster together (i.e., reefs were partitioned at terminal nodes by reef name and not by survey time), suggesting that high-frequency 
acoustic results were similar over time within reefs.

Physical parameters such as depth and rugosity and management parameters such as degree of protection were excluded from this CART analysis because their inclusion would result in the presence of a categorical, reef-specific proxy, which would mask biological variability within and among reefs and therefore confound the results.

Reefs were selected based on initial surveys to cover as broad a gradient in benthic cover and fish density as possible. While reefs varied appreciably in benthic cover, fish species assemblages proved to be more similar among reefs than was originally desired. Nevertheless, reef fish species assemblages did vary within reefs among survey periods. Because there was no clear indication of how fast those changes took place, care was taken to relate visual survey data to acoustic data only in months where the 2 datasets overlapped.

Similarly, there was variability over time in the acoustic data. While wind speed did not seem to correlate with acoustic data here, such correlations have been identified in other studies (e.g., Staaterman et al., 2014). The lack of correlation noted here could be a result of the fact that wind speed data were obtained from a NOAA buoy in Kahului Harbor, near but not directly adjacent to any of the recording sites. Alternatively, these reefs, many of which were close to shore, were somewhat protected from winds, which would suggest that soundscape parameters were driven by other factors.

Temperature did correlate with high-frequency sound levels, which were primarily driven by snapping shrimp activity, but the magnitude of this relationship varied among reefs. While consistent with other studies (Kaplan et al., 2015), the causal link between temperature and snapping shrimp activity has yet to be fully elucidated. Further work should attempt to better understand the drivers of this relationship. The negative correlation noted at MM17 could be a result of early arrival of humpback whale song in the fall months (i.e., before the December cutoff after which low-frequency recordings were not considered).

Some acoustic variability over longer time scales could not be satisfactorily explained. For example, at Ahihi there appeared to be a shift by 2-3 dB after instrument redeployment in July 2015 at high- but not low-frequency. This frequency-dependence effectively rules out recorder deviance, but because this shift was not visible at other adjacent reefs, it is similarly unlikely that there was a true increase in snapping shrimp activity at this time. Perhaps the most parsimonious explanation is a change in instrument orientation, which could explain why only high-frequency levels increased given their higher directionality compared to low- 
frequency sounds.

Lunar and diel periodicity in biological sound pressure level, which has been extensively described elsewhere (Staaterman et al., 2014; Kaplan et al., 2015), was also evident here in both frequency bands and at all reefs, with the exception of MM17, the sandy control site, where only limited and low-amplitude variability was evident. While diel periodicity appeared to be much greater in magnitude than lunar periodicity, the strength of both of these trends varied among reefs. Because these differences could reflect underlying differences in the sound-producing species assemblages present, these data were used as the response variables in the CART regression (after distillation into the first principal component).

Assessing amplitude variability over time is not, on its own, sufficient to fully capture the complex variability in sound production. Accordingly, this study developed a new metric to capture both spectral and amplitude variability within short recordings to better quantify biological sound production. This metric is based on calculating the median absolute deviations in $\mathrm{dB}$ within a recording and octave band and then averaging across octave bands. Accordingly, recordings with low spectral and amplitude variation will correspond to a lower MAD value than recordings with high spectral or amplitude variation, which could result from elevated levels of biological sound production. Unlike some other acoustic metrics, which present results in arbitrary (and therefore difficult to understand and compare) units (e.g., Bertucci et al., 2016), MAD values are expressed in $\mathrm{dB}$ and directly reflect acoustic variability within a recording.

Because MAD is calculated based on deviations from a median value, there are, expectedly, differences between the trends in diel periodicity in MAD and in SPL. For example, while peaks at dusk and dawn are evident in both metrics for some reefs such as Olowalu and Red Hill at low frequencies, such a trend is not present in MAD at Honolua in that frequency band. The presence of these differences underscores the value in having more than 1 metric. 


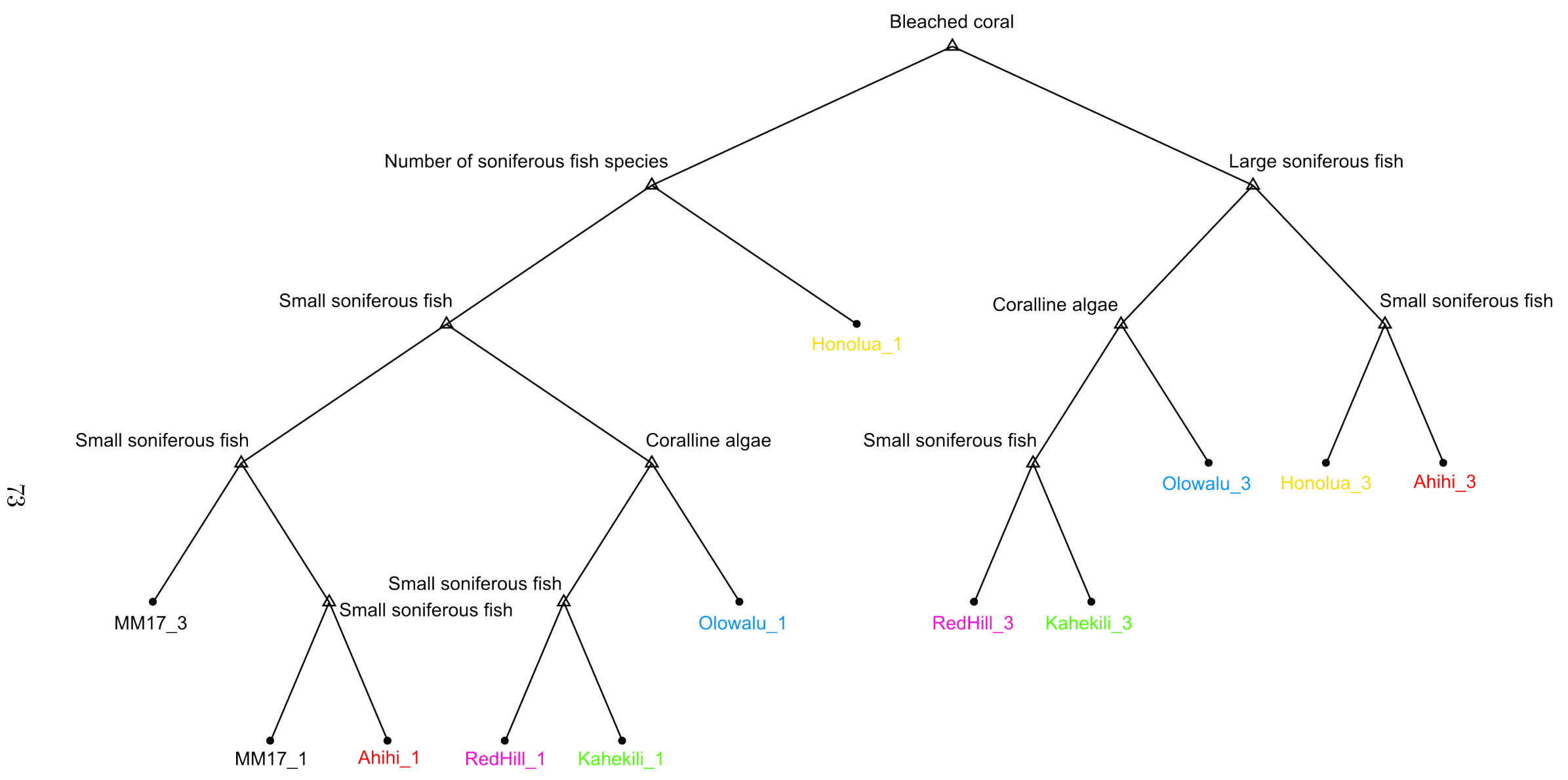

Figure 3.12: Regression tree for low-frequency acoustic parameters showing the relationship among reefs and biological variables in September 2014 (sample 1) and October 2015 (sample 3). Data for Molokini were unavailable at both sampling periods as a result of acoustic recorder malfunction. There was a clear split between sampling periods for all reefs except MM17. This split was mediated at the highest level by the percent cover of bleached coral. The most important predictor variables were the number of soniferous fish species and the percent cover of turf algae (Figure 3.14). Branches indicate binary splits in a given variable (identified at the branch) used to segment among reefs and the terminal nodes are the reefs with each number noting the sampling period. 


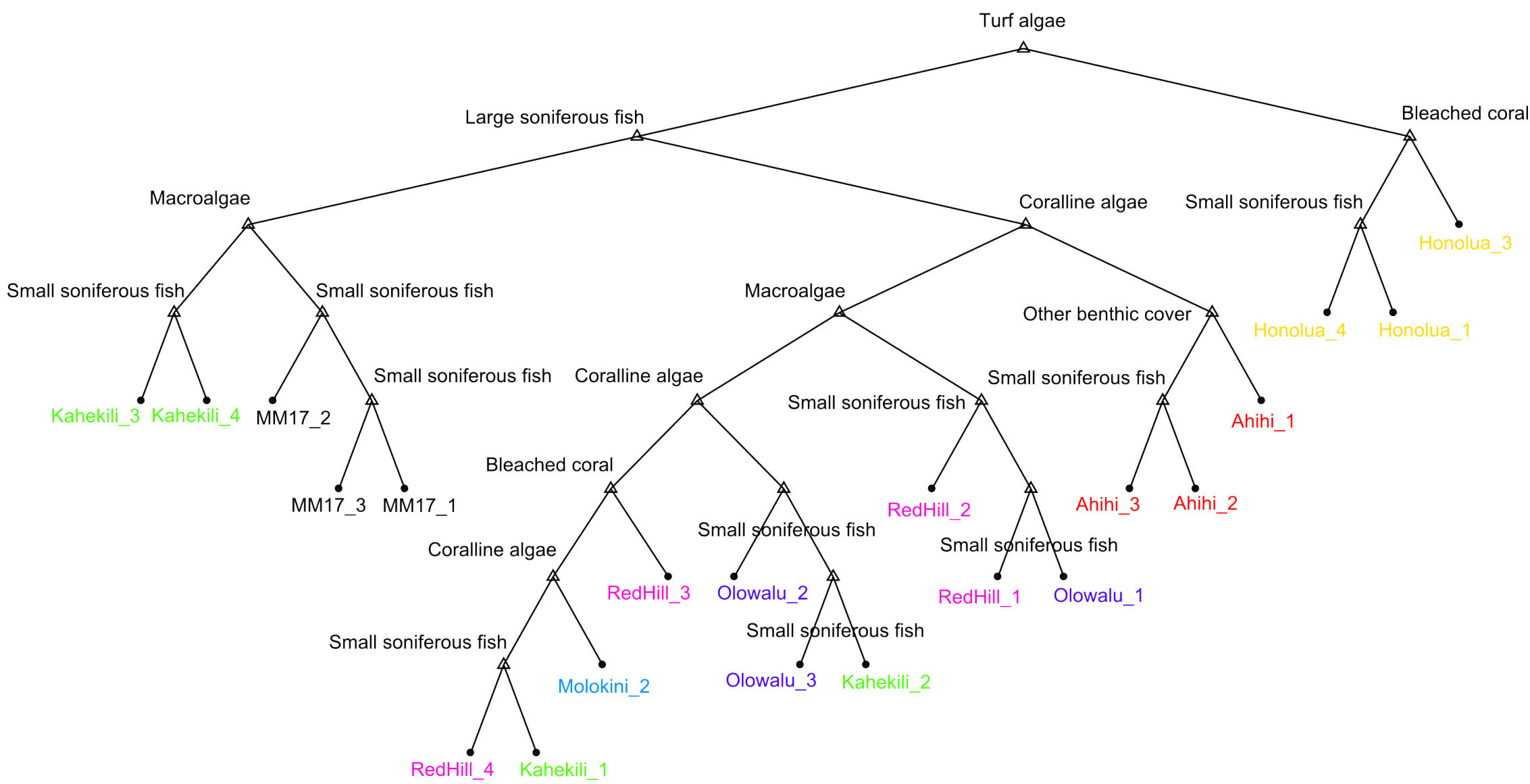

Figure 3.13: Regression tree for high-frequency acoustic parameters showing the relationship among reefs and biological variables in September 2014 (sample 1), February/March 2015 (sample 2), October 2015 (sample 3), and January 2016 (sample 4). Some data are missing for certain reefs in each sampling period. While MM17 and Honolua tended to group, there was considerable overlap between Red Hill, Olowalu, Kahekili, and Molokini, suggesting that the snapping shrimp acoustic activity was similar among these reefs. The most important predictor variable was the percent cover of turf algae (Figure 3.14). Branches indicate binary splits in a given variable (identified at the branch) used to segment among reefs and the terminal nodes are the reefs with each number noting the sampling period. 

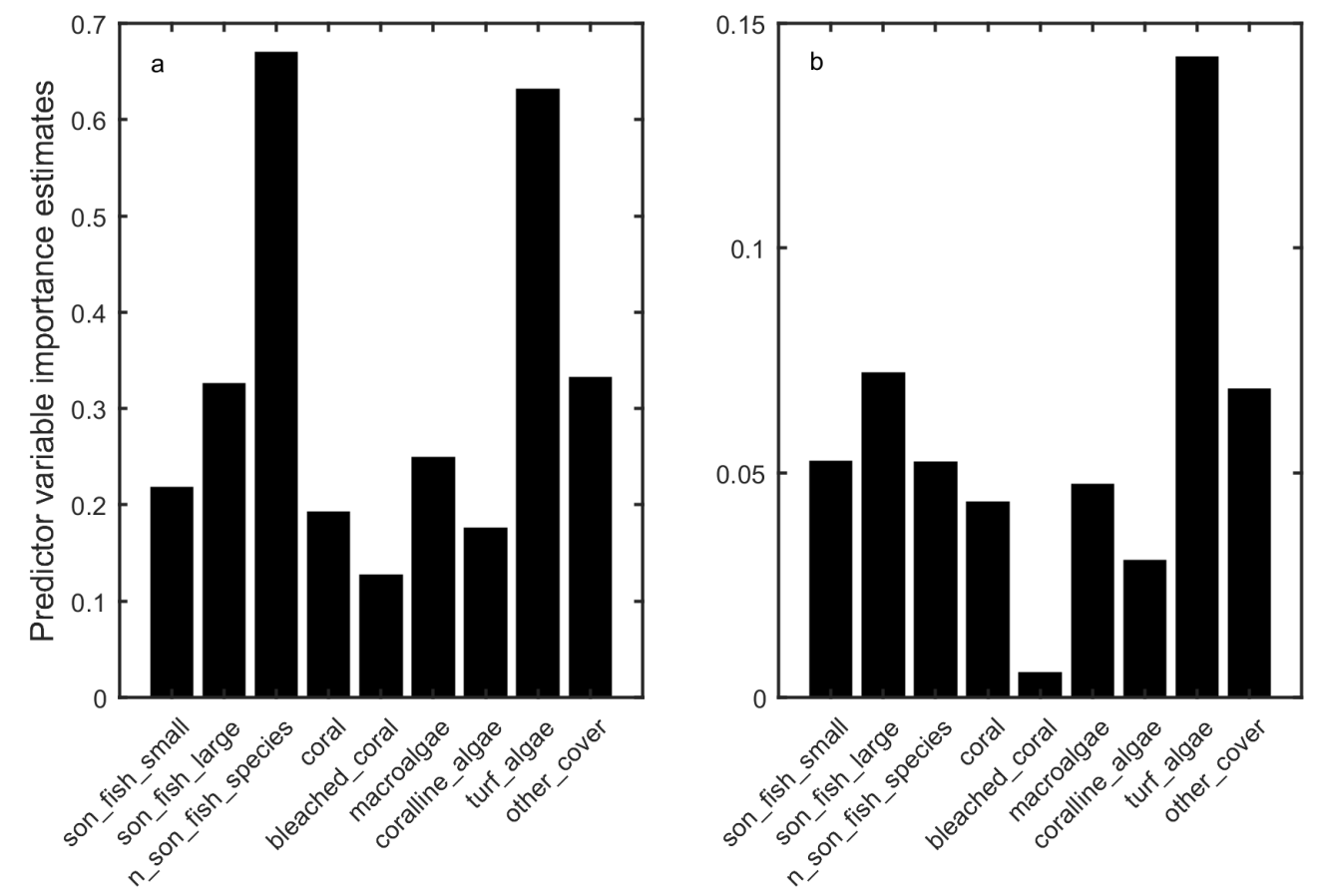

Predictors

Figure 3.14: Variable importance for the (a) low- and (b) high-frequency regression trees (Figures 3.12-13). In both cases, turf algae was important. For the low-frequency band, the number of soniferous fish species was also important. Son refers to soniferous; small is less than $15 \mathrm{~cm}$ total length, and benthic parameter units are in percent cover.

Indeed, based on the results from these 2 metrics, Honolua stands out as a unique reef. Shrimp snaps appear to extend into much lower frequencies than at other reefs, which is suggestive of clipping despite the fact that these files did not contain voltage values of \pm 1 . Nevertheless, this low-frequency energy, whether spurious or otherwise, is likely responsible for the divergence in diel trends in SPL and MAD seen at Honolua compared to the other study sites. While the reasons for the prevalence of high-amplitude shrimp snaps at Honolua compared to other reefs are not obvious, it is possible that bathymetry plays a role. Honolua has the highest rugosity of any studied reef, and accordingly this extreme in three-dimensional structure may provide additional snapping shrimp habitat at Honolua, which could allow the snapping shrimp to reside closer to the recorder than would have been possible at other reefs, thereby increasing the received snap levels (and potentially resulting in clipping).

In conclusion, this study demonstrates that, despite the considerable variability in bio- 
logical sound production within and among reefs, low-frequency sound levels within reefs varied over time whereas high-frequency levels were more similar within reefs over time. The most distinct reefs tended to cluster separately in the regression trees; however, some other reefs that were similar in their species assemblages were too acoustically similar to be discriminable. Thus, inverse prediction of species assemblages using the acoustic parameters employed here was not possible. 


\subsection{Supplementary Figures}

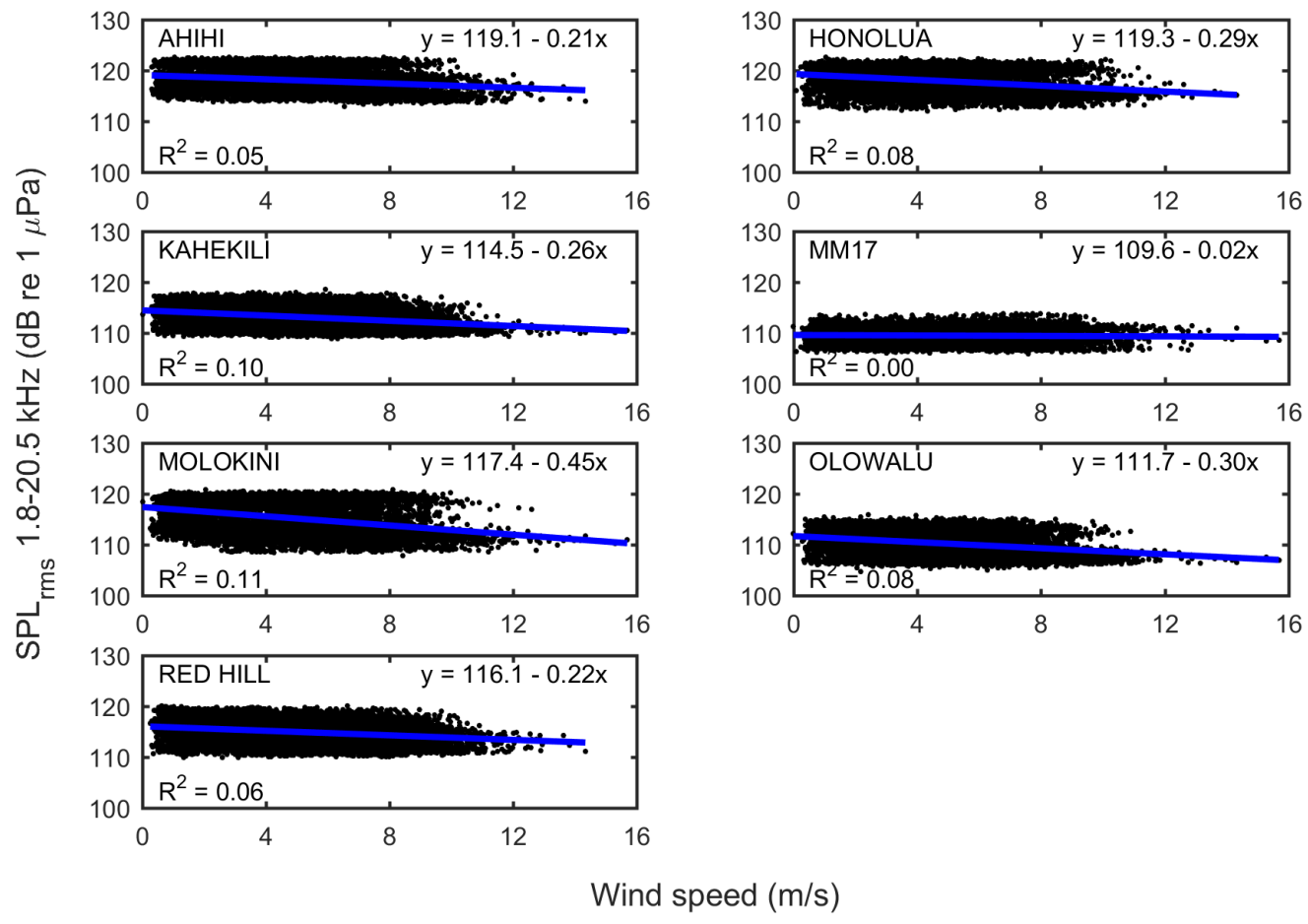

Figure 3.S1: Correlations between wind speed and high-frequency SPL at each study reef. Wind speed did not appear to have any relationship with these sound levels. 


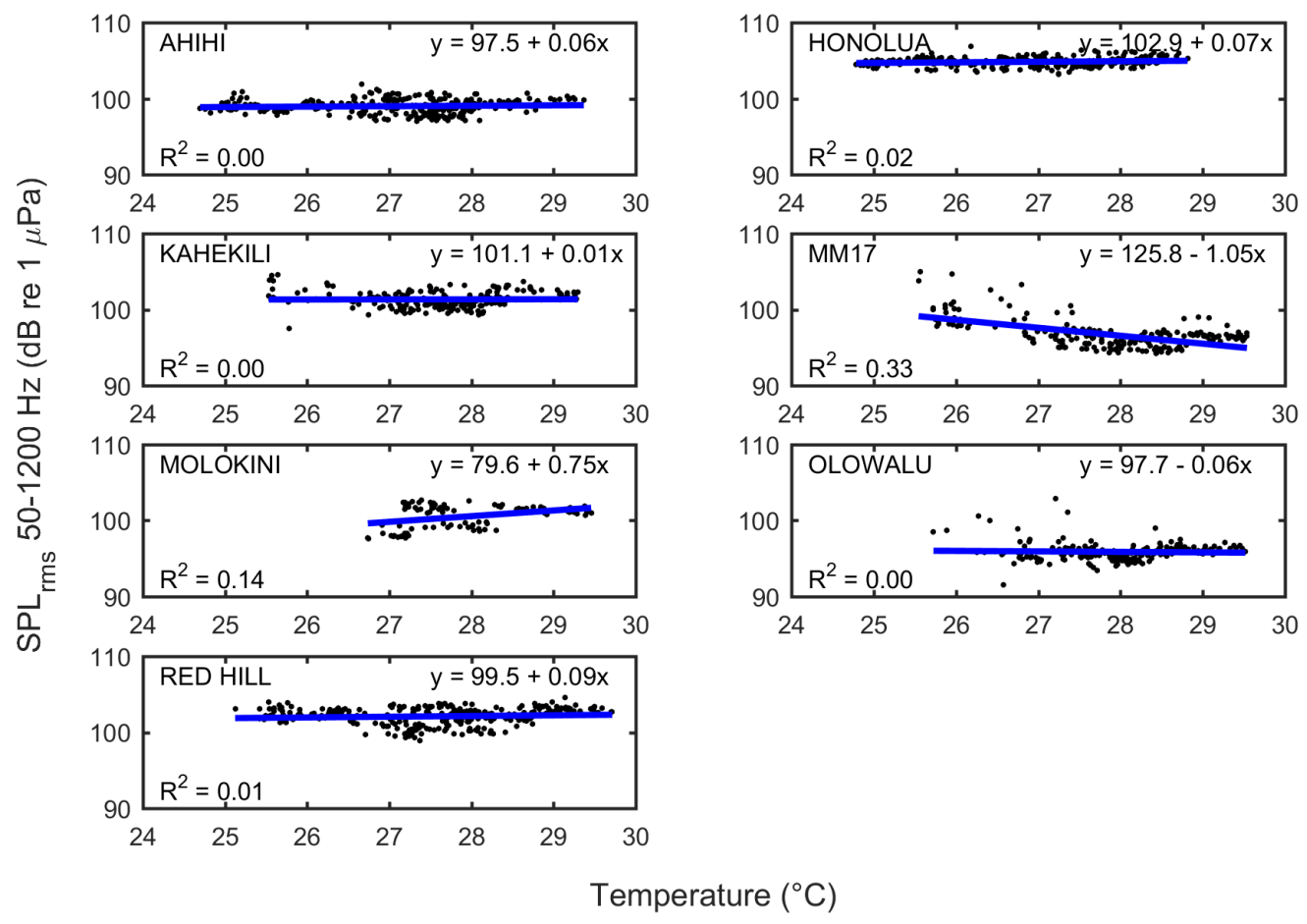

Figure 3.S2: Correlations between daily average temperature and low-frequency SPL for each study reef. There did not appear to be a link between water temperature and low-frequency SPL. 


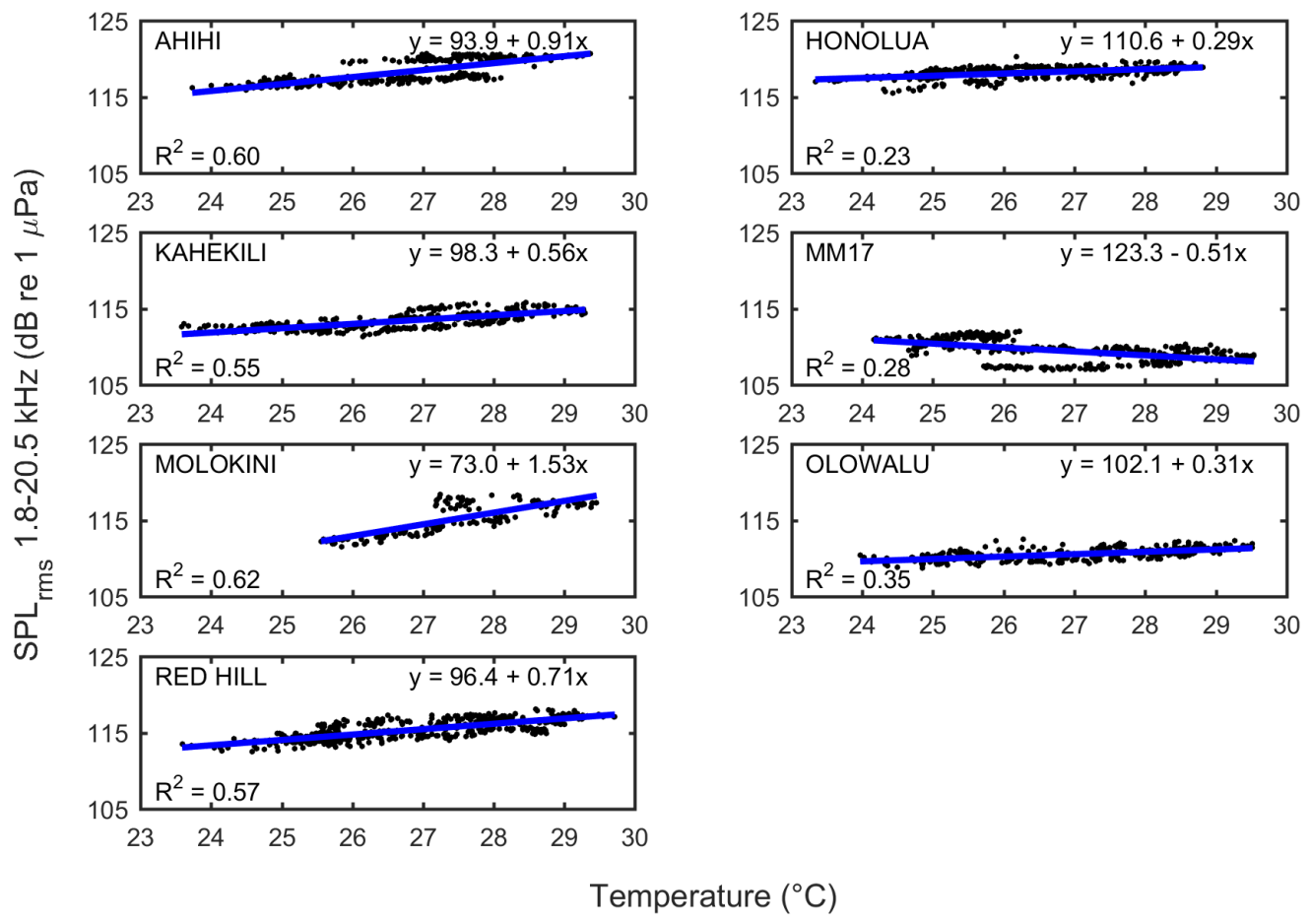

Figure 3.S3: Correlations between daily average temperature and high-frequency SPL. Strong positive correlations were evident at every reef except MM17, which was the sandy control (i.e., non-reef) site. The double band of SPL values at Ahihi is likely a result of the elevated levels seen in the final EAR deployment (July 2015) compared to earlier deployments (see Discussion for further details). 
THIS PAGE INTENTIONALLY LEFT BLANK 


\section{Chapter 4}

\section{Coral reef soundscapes may not be detectable far from the reef}

This chapter was originally published as: Kaplan, M.B. \& Mooney, T.A. 2016. Coral reef soundscapes may not be detectable far from the reef. Scientific Reports 6:31862. DOI: $10.1038 /$ srep31862. Used with permission as granted in the original copyright agreement. 


\subsection{Abstract}

Biological sounds produced on coral reefs may provide settlement cues to marine larvae. Sound fields are composed of pressure and particle motion, the latter of which is the back and forth movement of acoustic particles. Particle motion (i.e., not pressure) is the relevant acoustic stimulus for many, if not most, marine animals. However, there have been no field measurements of reef particle motion. To address this deficiency, both pressure and particle motion were recorded at a range of distances from a Hawaiian coral reef at dawn and mid-morning on 3 separate days. Sound pressure attenuated with distance from the reef at dawn. Similar trends were apparent for particle velocity but with considerable variability. In general, average sound levels were low and perhaps too faint to be used as an orientation cue except very close to the reef. However, individual transient sounds that exceeded the mean values, sometimes by up to an order of magnitude, might be detectable far from the reef, depending on the hearing abilities of the larva. If sound is not being used as a long-range cue, it might still be useful for habitat selection or other biological activities within a reef.

\subsection{Introduction}

Coral reefs are hubs of marine biodiversity, and recruitment to these reefs provides the foundation of these ecosystems. Larval animals seeking the reef for settlement may use a variety of cues (Kingsford et al., 2002). A range of sounds are produced on reefs, and it is increasingly realized that these acoustic components may assist pelagic larvae in locating and orienting toward suitable juvenile and adult habitat (Simpson et al., 2004, 2005; Vermeij et al., 2010). For example, experimental moorings that broadcasted reef sound attracted more fish larvae than silent controls (Simpson et al., 2004). Similarly, some juvenile fish species preferentially selected patch reefs broadcasting reef sound (Radford et al., 2011a). Reef sound has also been shown to induce metamorphosis in preparation for settlement in some crab larvae (Stanley et al., 2009).

It has been suggested that these reef soundscapes may offer a reef orientation cue at relatively large distances compared to some other stimuli (Kingsford et al., 2002; Atema, 2012). Loud choruses of reef sounds may be detectable from hundreds to thousands of meters from the reef (Radford et al., 2011b; Mann et al., 2007; Cato, 1980). A study by Egner and Mann (2005) proposed that sergeant major damselfish (Abudefduf saxatilis) would likely be 
able to use coral reef sounds as a significant navigation cue up to $500 \mathrm{~m}$ away. For some crab species, orientation and settlement responses were estimated at up to $40 \mathrm{~km}$ (Stanley et al., 2011). However, there are few studies quantifying the range over which reef sounds may actually propagate. This paucity of data limits our understanding of the extent to which this cue could be used by settling larvae. Furthermore, many sounds on the reef, such as fish choruses or snapping shrimp snaps, are produced with temporal periodicity (Staaterman et al., 2014; Kaplan et al., 2015). This variation in sound levels suggests that the distances over which reef sound is detectable may also vary with time.

The ability of a given larva to detect sound cues will depend on its hearing abilities. While hearing has been studied in only a few of the approximately 12,000 extant marine fish species that live in shallow water or close to shore, and for whom acoustic settlement cues might be relevant (Ladich and Fay, 2013; Helfman et al., 2009), those that have been studied generally show best sensitivities to low-frequency sound (Ladich and Fay, 2013). For example, juvenile sergeant major damselfish (A. saxatilis) were most sensitive to $100-400 \mathrm{~Hz}$ sounds in a hearing test (Egner and Mann, 2005). No hearing studies have been carried out on larval marine invertebrates. Audiometric studies on adults are also somewhat limited, but like most fish, those tested (e.g., cephalopods and crustaceans) show lowest (most sensitive) thresholds below 1000 Hz (Samson et al., 2014; Mooney et al., 2010; Budelmann, 1992a,b; Goodall et al., 1990). Thus, it is critical to measure the propagation of reef sound at a range of frequencies, and particularly those low-frequency sounds that are within the hearing ranges of many marine fishes and invertebrates.

Sound is a mechanical disturbance that can be described in terms of its pressure or particle motion (i.e., velocity or acceleration) (Medwin and Blue, 2005). Most fish use their otolith system to detect the back-and-forth, vibratory-like, particle motion component of sound, whereas those with a swimbladder close to or connected to the ear can also detect sound pressure via auditory specializations (Popper and Fay, 2011). Auditory physiological and morphological investigations of invertebrates indicate that those tested appear to be almost exclusively sensitive to particle motion (i.e., not pressure) (Mooney et al., 2010; Goodall et al., 1990; Packard et al., 1990).

Bioacoustic larval settlement studies (Simpson et al., 2004, 2005; Radford et al., 2011a; Stanley et al., 2011; Parmentier et al., 2015) and investigations of the distances over which reef sound cues may travel have measured those cues in terms of pressure (Radford et al., 
2011b; Piercy et al., 2014), leaving responses to particle motion poorly understood. For example, Radford et al. (2011b) modeled a reef as an extended sound source and found that reef sound pressure propagates much further than predicted by a point source. However, without acoustic particle motion data, the sound cues available to settling larvae remain largely unknown. Furthermore, the relationship between sound pressure and particle motion is not necessarily predictable close to the source in the near field (Merchant et al., 2015; Nedelec et al., 2016). Accordingly, to understand both pressure and particle motion cues, direct measurements are required. In addition, particle motion is inherently directional and thus can be used to identify the axis of a sound source, which is a vital attribute for closely spaced, low-frequency "ears" such as fish otoliths and invertebrate statoliths.

Indeed, there appear to be no direct measurements of reef particle motion in field conditions, limiting any estimation of the actual levels and ranges over which these settlement signals might be detectable. Nor are there data that quantify how this signal might vary in time. To address this deficiency, we carried out acoustic recording transects measuring both the particle motion and pressure components of reef sound at multiple distances from the reef. Measurements were made at 2 times of day (dawn and mid-morning) and repeated on 3 separate days to determine how the reef soundscape might change with time of day. Measurements were made in the summer (July 2015), coinciding with periods of fish and coral settlement (Walsh, 1987; Richmond and Hunter, 1990).

\subsection{Methods}

This study was conducted on July 17, 19, and 20, 2015, immediately after the new moon at a southwest-facing reef, Olowalu, which is located on Maui's (HI, USA) leeward side $\left(20.802^{\circ}\right.$ $\left.\mathrm{N}, 156.618^{\circ} \mathrm{W}\right)$. During the data collection period, sea state on all 3 of these days was less than Beaufort 2 (wind $4-6$ knots, wave heights $0.2-0.5 \mathrm{~m}$ ). Olowalu is a broad, relatively flat reef dominated by branching corals with some massive corals and sand channels. Much of the reef is found from approximately 6-11 $\mathrm{m}$ depth. This reef has approximately $70 \%$ live coral cover, and 33 fish species were observed in visual surveys (unpublished data, February 2015). The benthic cover changes from coral-dominated structure to sand with increasing distance from the reef.

Acoustic data were collected as follows. At the start of the experiment, a stationary 
DMON digital acoustic recorder (Woods Hole Oceanographic Institution, Woods Hole, MA, USA) was deployed on a mooring on the reef (approximately $1 \mathrm{~m}$ off the bottom) to record sounds directly on the reef and monitor the natural temporal changes in sound production during the course of the study (Figure 4.1). The mooring was deployed at the same location as another acoustic recording device, which had been collecting data at this position for approximately 16 months as part of a separate study. This mooring DMON recorded using a low-frequency hydrophone element (Navy type II ceramic) with a high-pass filter at $8 \mathrm{~Hz}$ and $60 \mathrm{kHz}$ sample rate (down-sampled to $10 \mathrm{kHz}$ before commitment to onboard FLASH memory).

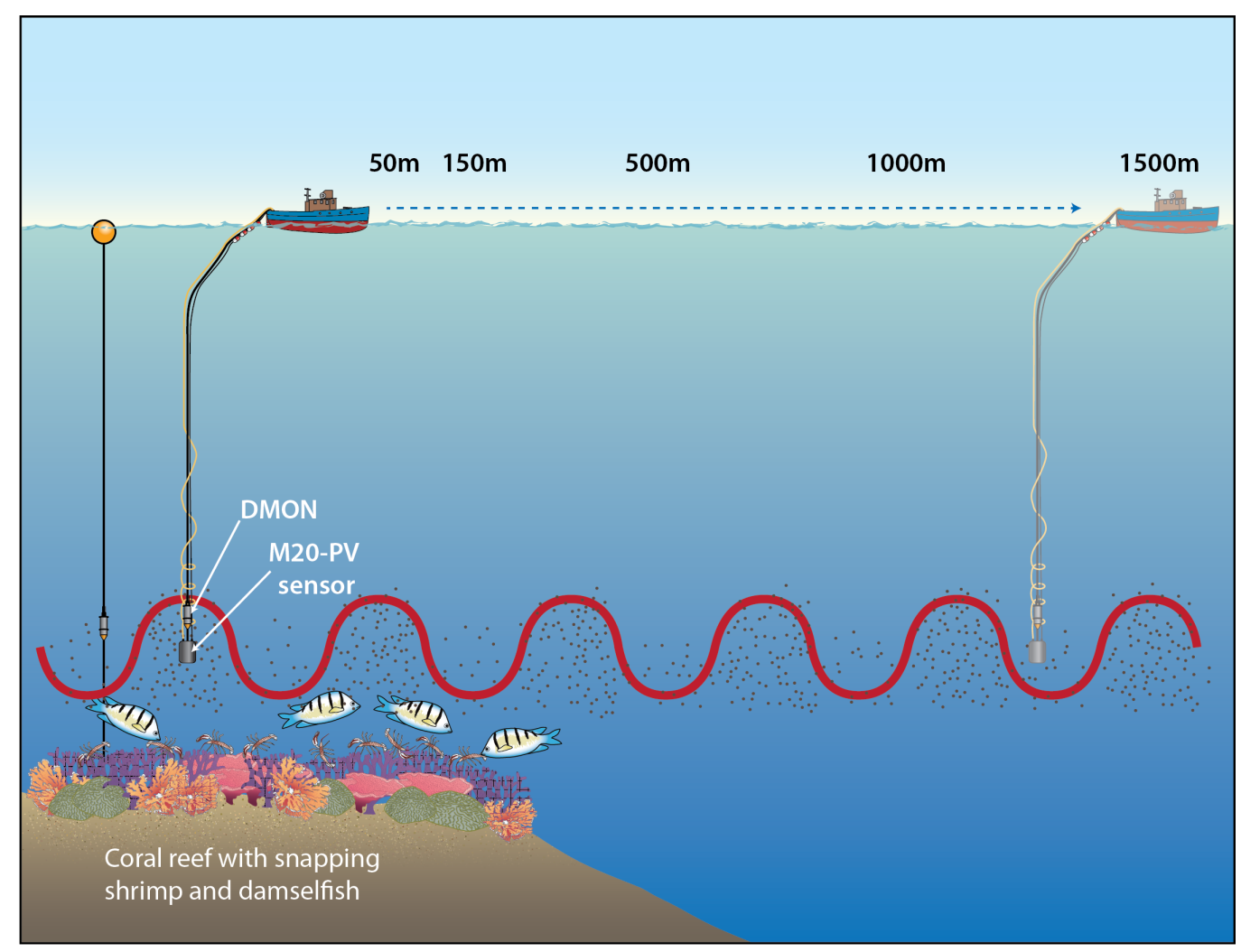

Figure 4.1: Schematic of the data collection transect. Acoustic recordings were collected on the reef with a DMON affixed to the mooring on the left and from the vessel using both a DMON and the M20 particle velocity sensor. Image courtesy of Jack Cook, WHOI Graphic Services

To measure the reef sound propagation, vessel-based transects were conducted, recording reef sounds at various distances from the reef. The vessel-based recording package consisted of 2 instruments: a DMON set up as above to measure sound pressure in a manner identical to the mooring, and an M20 particle velocimeter (GeoSpectrum Technolo- 
gies Inc, Dartmouth, NS) to record particle velocity (i.e., the velocity component of acoustic particle motion). The M20 contains 3 vector sensors (accelerometers), an omnidirectional hydrophone, and a digital magnetometer/accelerometer chip for instrument orientation sensing, all housed in a negatively buoyant aluminum can. The M20 has a usable frequency range of up to $3 \mathrm{kHz}$; accordingly, sample rates of either 6000 or $12500 \mathrm{~Hz}$ were used for all 4 channels, with data recorded directly onto a laptop via a data acquisition card (USB6002; National Instruments Corporation, Austin, TX), using a customized Matlab (The Mathworks, Natick, MA) program. The instruments were deployed at approximately $10 \mathrm{~m}$ depth (the maximum possible depth given the depth of the reef) on a mooring consisting of 2 communication cables married to a 1/4-inch polypropylene line. An Anchor Buddy (Greenfield Products, Greenfield, $\mathrm{OH}$ ) was fastened to the top and bottom of the mooring setup to dampen wave-driven vertical movements. The M20 was affixed by eye bolt to the bottom of this line with the DMON slightly above it, oriented in a hydrophone-down position. A 2-lb lead weight was affixed to the line adjacent to the DMON (i.e., above the M20) to help ensure a vertical mooring orientation. At the surface, 12 1-lb gillnet floats were affixed to the line in an effort to further dampen wave activity.

Table 4.1: Summary of point data collection combined among perpendicular and parallel transects.

\begin{tabular}{l|l|l|l|l} 
Date & Start time (local) & End time (local) & $\begin{array}{l}\text { Number of points } \\
\text { on transect }\end{array}$ & $\begin{array}{l}\text { Number of points } \\
\text { after boat noise } \\
\text { exclusion }\end{array}$ \\
\hline $17 / 07 / 15$ & $5: 01$ & $6: 51$ & 15 & 14 \\
$17 / 07 / 15$ & $9: 01$ & $9: 53$ & 8 & 2 \\
$19 / 07 / 15$ & $5: 01$ & $5: 58$ & 8 & 8 \\
$19 / 07 / 15$ & $9: 40$ & $10: 27$ & 8 & 4 \\
$20 / 07 / 15$ & $4: 55$ & $6: 03$ & 10 & 9 \\
$20 / 07 / 15$ & $8: 57$ & $9: 48$ & 10 & 8
\end{tabular}

Recordings were made at specific points (Figure 4.2) along 2 transects, 1 perpendicular and 1 parallel to the reef at 2 times of day - dawn and mid-morning. First, the perpendicular transect from the reef out to approximately $1500 \mathrm{~m}$ was carried out, followed by the parallel transect. On the perpendicular transect, at minimum recordings were made on the reef and at 50,150, 500, 1000 and $1500 \mathrm{~m}$ away from the reef. On July 17 and July 20 some other locations were sampled, but those were excluded from subsequent analyses because of the lack of multiple days of recording at those positions (Table 4.1). On the parallel transect, 
2 points were selected, 1 north and 1 south of the perpendicular transect. These points were both parallel with the $500 \mathrm{~m}$ position of the perpendicular transect but were 360 and $349 \mathrm{~m}$ away respectively from the projected tip of the reef (based on map data; see Figure 4.1). Recording duration at each point was $2.5 \mathrm{~min}$ and the recordings were made with the vessel engine off to minimize acoustic and physical disturbance to the acoustic recording instruments. Each set of transects took approximately 60 min to complete (except for the first dawn transect, during which several additional recordings were made). Water depth along the transect varied from approximately $11 \mathrm{~m}$ at the reef to approximately $60 \mathrm{~m}$ at the $1500 \mathrm{~m}$ position.
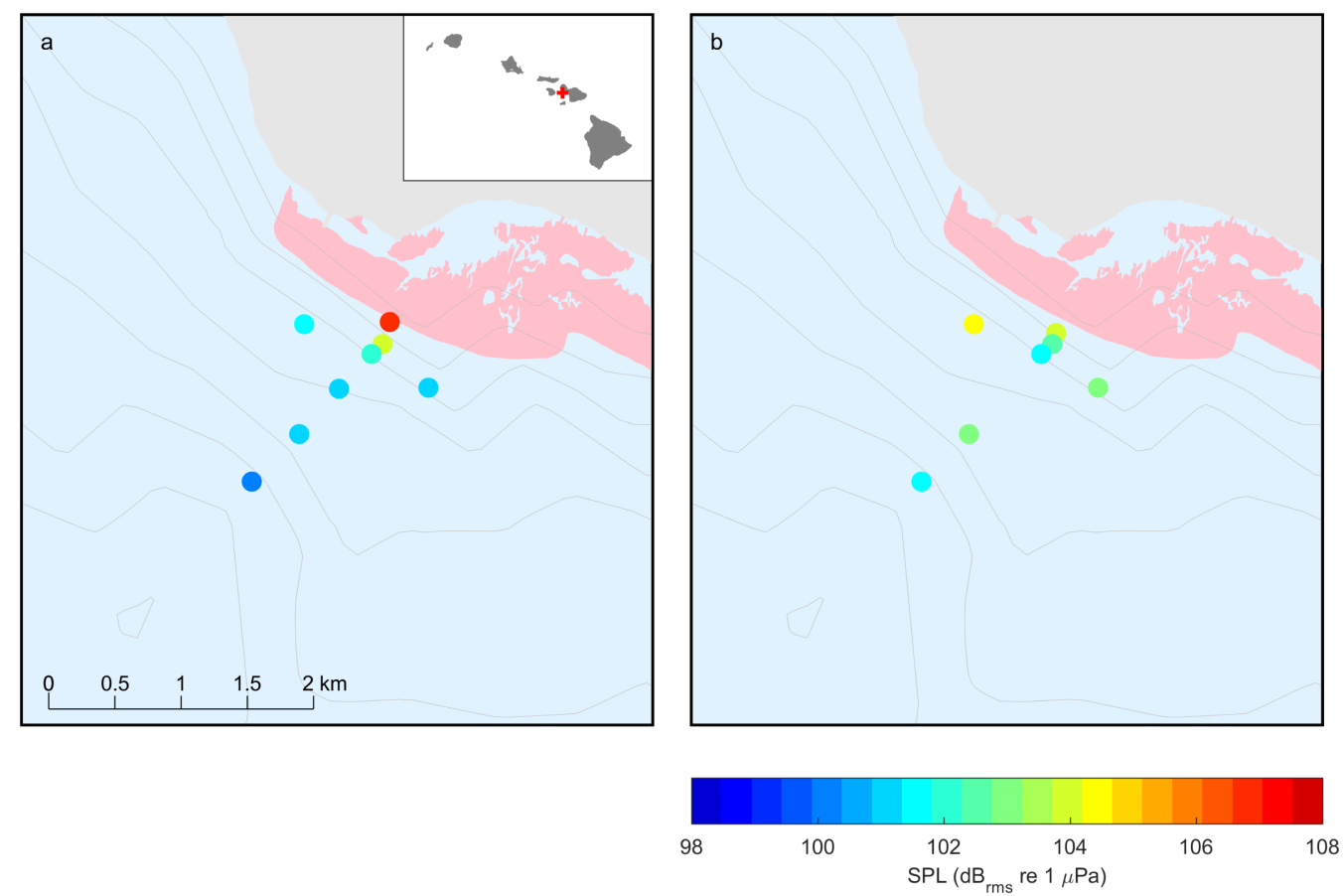

Figure 4.2: Map of Olowalu reef and transect point sound pressure levels $(200-4900 \mathrm{~Hz})$ at different distances from the reef during the (a) dawn and (b) mid-morning transects. Sound levels decreased with distance from the reef during the dawn transect, but such a trend was not as obvious for the mid-morning transect. The pink shaded area denotes coral cover and the contour lines depict $10 \mathrm{~m}$ increments.

All acoustic analyses were carried out in Matlab 8.6. Each recording from both DMONs and the M20 was reduced into a 1-minute segment to remove any extraneous noise associated with deployment. Any segments containing vessel noise were excluded from subsequent 
analyses; however, segments with limited wave noise were retained. The remaining segments were detrended to remove any DC offset, corrected for instrument sensitivity, and band pass filtered at 200-4900 Hz (full band DMON), 200-1000 Hz (low-frequency (LF) band DMON), 2-4 kHz (high-frequency (HF) band DMON), and 200-2800 Hz (M20) using 4pole Butterworth filters. The high pass filter $(200 \mathrm{~Hz})$ for all bands was set to ensure that any low-frequency periodic signals from physical motion (e.g., surface waves) were significantly (i.e., orders of magnitude) below the filter's frequency.

Sound pressure levels (SPL; root-mean-square) and power spectral density estimates (FFT size: 512; Hann window, $75 \%$ overlap) were calculated for the entire 1-minute segment of the DMON hydrophone recordings. In addition, SPL was also calculated in $5 \mathrm{~ms}$ windows in the DMON LF and HF band to examine variability within the minute-long records.

Mean particle velocity measurements and power spectral density estimates (same spectral settings as above) were calculated in the $200-2800 \mathrm{~Hz}$ band for each of the M20 accelerometer axes. As a result of a sensor orientation chip malfunction, it was not possible to correct the particle velocity axes with measured pitch, roll, and heading. Nevertheless, because the bottom of the mooring was weighted, the $\mathrm{z}$ axis can be reliably referred to as the vertical axis and the $\mathrm{x}$ - and $\mathrm{y}$-axes as the horizontal axes. Accordingly, the $\mathrm{x}$ - and $\mathrm{y}$-axes were combined so as to present a total magnitude of horizontal velocity.

Attenuation with distance from a source is a result of spreading loss and absorption, the latter of which is negligible at these frequencies and distances (Zimmer, 2011). Spreading loss can be modeled as spherical $\left(20 \log _{10} R\right)$ or cylindrical $\left(10 \log _{10} R\right)$ spreading, where $R$ is range from the source in meters. These estimated losses were compared to the measured changes in sound pressure level with distance from the reef. To quantify the in situ spreading, exponential decay functions were fitted to the sound pressure and particle velocity attenuation data.

Particle acceleration, the temporal differentiation of particle velocity, can be found using the relationship $A=2 \pi \times f \times v$, where $A$ is the particle acceleration, $f$ is frequency and $v$ is particle velocity. This was done with each axis individually to compare measured values to published acceleration-based hearing thresholds of fishes and invertebrates. To focus on the diel variability in sound production and attenuation with distance from the reef, mean values across days for each transect position and time of day are presented with standard deviations (both calculated on the linear scale). Only positions on the transect that were 
recorded more than once are reported here.

\subsection{Results}

Sound pressure levels were generally highest on the reef and attenuated with distance during the dawn transect; such attenuation was not detectable during the mid-morning transect (Figure 4.2; Table 4.2). On-reef levels stayed relatively constant over the course of the dawn transect, with 1 exception (c. $400 \mathrm{~m}$, Figure 4.3a) where levels were lower during the parallel transect. Full-band and low-frequency transect levels at dawn appeared to decrease along the transect with distance from the reef (Figure 4.3a). On-reef levels were more variable during the mid-morning and decreased over the course of the transect (Figure 4.3b). The mid-morning full-band transect levels were more variable than those at dawn and did not appear to decrease with distance from the reef. High-frequency sound pressure levels (SPL) did decrease with distance from the reef in both transects; however, because the levels were much lower than the low-frequency SPL, their contribution to the overall trend was limited. Indeed, low-frequency SPL tracked the full-band levels very closely as the low-frequency components contributed the majority of the energy to the full band. Attenuation of both the dawn and mid-morning transect sound level measurements was lower (more gradual) than was predicted by standard spherical or cylindrical spreading (Figure 4.3c,d).

Power spectra followed a roughly similar shape across transect positions and time of day, with elevated low frequencies (less than $1000 \mathrm{~Hz}$; Figure 4.3e,f). These low frequencies attenuated with distance from the reef during the dawn transect. Such attenuation was again not apparent in the mid-morning transect, reflecting the fact that sound levels were lower. On-reef positions had elevated higher-frequency levels $(2-4 \mathrm{kHz})$; during the dawn transect, sound levels were approximately $10 \mathrm{~dB}$ greater on the reef than the next closest position, while during the mid-morning on-reef levels were elevated but less so. These highfrequency, snapping shrimp components attenuated rather quickly immediately off the reef and then less so with increasing distance, which was expected given that attenuation rates increase with frequency. The position with the lowest high-frequency levels was furthest from the reef (1500 $\mathrm{m}$ distance).

Particle velocities in the horizontal (x-y) and vertical (z) axes appeared to decrease with distance from the reef during the dawn transect and in the horizontal axis only during the 

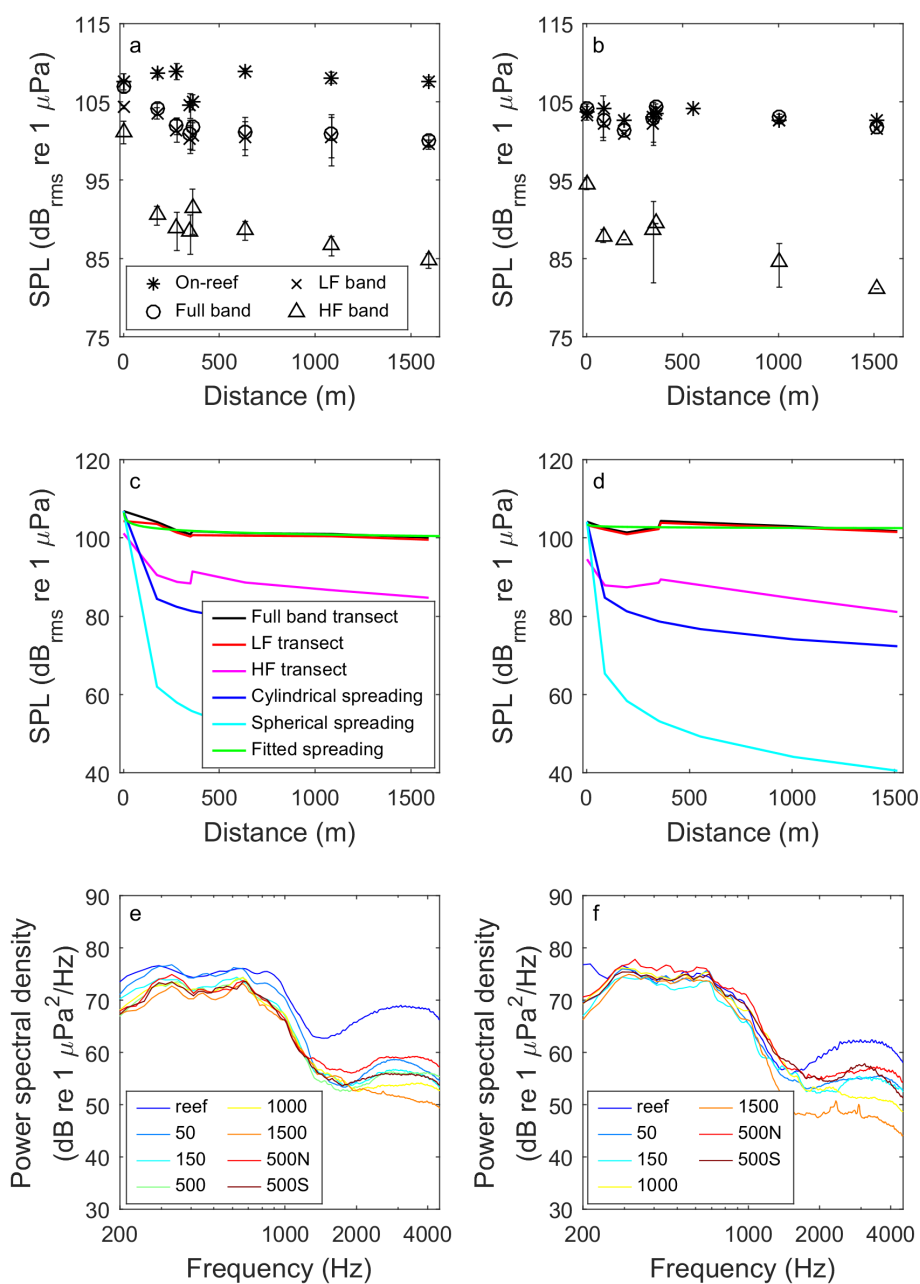

Figure 4.3: Mean $( \pm \mathrm{SD})$ sound pressure levels $(200-4900 \mathrm{~Hz})$ on the reef (full band) and low- (200-1000 Hz), high-frequency $(2000-4000 \mathrm{~Hz})$ and full-band SPL at various distances from the reef at dawn (a) and midmorning (b). Transect levels for the full band, low- (LF) and high-frequency (HF) shown with estimated cylindrical and spherical spreading loss and full band fit lines (c,d) and associated mean power spectra for each distance from the reef at the 2 times of day $(\mathrm{e}, \mathrm{f})$. On-reef sound levels stayed relatively constant during the dawn transect and varied over time during the mid-morning transect; conversely, fullband transect levels decreased with distance from the reef during the dawn but not the mid-morning transect. While high-frequency SPL attenuated with distance from the reef at both times of day, levels were considerably lower than the low-frequency SPL and accordingly make less of a contribution to the full-band trends. In neither case did attenuation match predicted spreading loss. High frequencies were elevated on the reef compared to every other transect point and decreased rapidly with distance from the reef. Low frequencies during the dawn transect appeared to decrease with distance from the reef while no such trend was evident for the mid-morning transect. No data were retained for the $500 \mathrm{~m}$ position during the mid-morning transect. See Table 4.3 for the equations of the fit lines in $(c, d)$. 
mid-morning transect, although there was considerable variability around the mean (Figure 4.4; Table 4.1). Notably, the vertical velocities were roughly the same magnitude as the horizontal velocities in both transects.

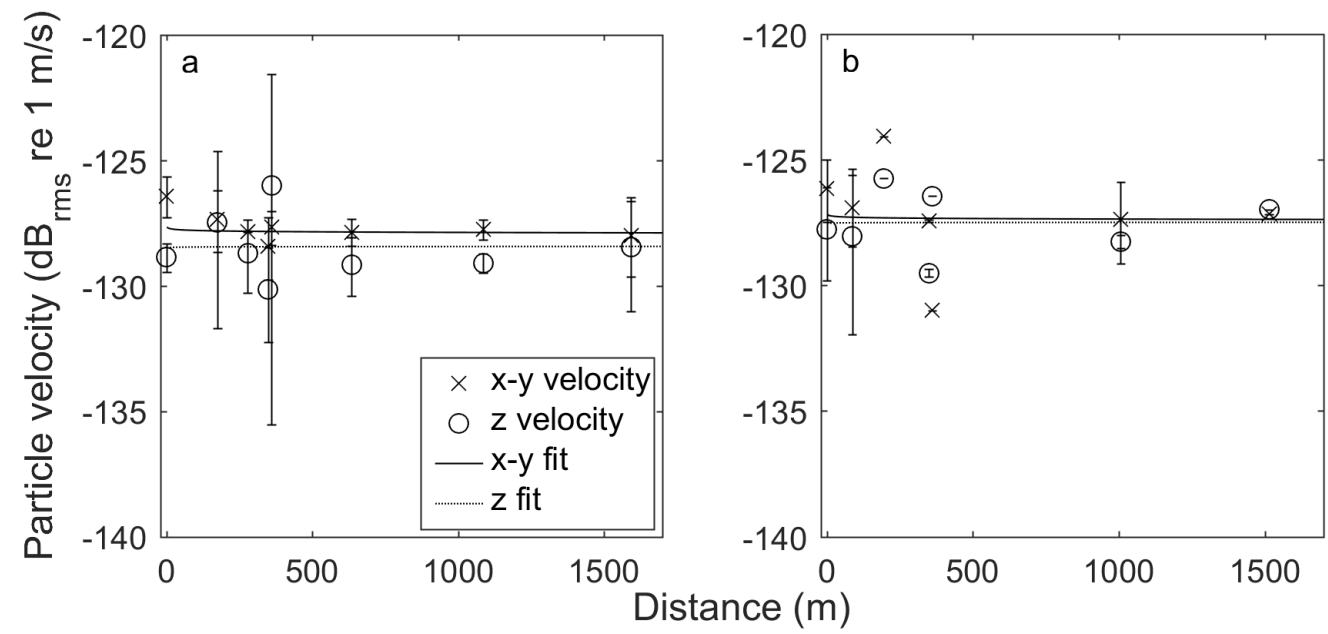

Figure 4.4: Mean ( \pm SD) particle velocities for the horizontal $(\mathrm{x}-\mathrm{y})$ and vertical $(\mathrm{z})$ axes of the M20 at a range of distances from the reef. Velocities in both major axes appeared to decrease with distance from the reef during the dawn (a) transect and in the $x-y$ axes during the mid-morning (b) transect, but there was considerable variability. Vertical velocities were nearly as high as horizontal velocities for both transects. No data were available for the 500 $\mathrm{m}$ position during the mid-morning transect. See Table 4.3 for equations of the fit lines.

Empirically derived spreading relationships for sound pressure and particle velocity in the horizontal and vertical axes were only statistically significant during the dawn transect for sound pressure and velocity in the $\mathrm{x}-\mathrm{y}$ axis (Table 4.3).

Acceleration values in the $\mathrm{x}$-axis were elevated at frequencies below $500 \mathrm{~Hz}$ in the dawn and mid-morning transects but did not follow a clear pattern with distance from the reef (Figure 4.5). Similar patterns were seen in the y and $\mathrm{z}$ axes (not shown). The transect position with the highest low-frequency acceleration values was the $500 \mathrm{~N}$ position during the dawn transect and the $150 \mathrm{~m}$ position during the mid-morning transect, although in both cases levels were similar across positions and times of day.

Median sound levels based on $5 \mathrm{~ms}$ segments of each recording pooled across days showed a similar trend to the averages with respect to attenuation with distance (Figure 4.6). However, there were a considerable number of high-amplitude outliers for both the dawn and mid-morning transect and for both the low- and high-frequency bands, although there were more outliers in the low-frequency band. 
Table 4.2: Mean sound pressure and particle velocity values by transect and position. SPL in $\mathrm{dB}$ re $1 \mu \mathrm{Pa}$ and $\mathrm{PV}$ in $\mathrm{dB}$ re $1 \mathrm{~m} \mathrm{~s}^{-1}$. DMON and M20 values are full band (200-4900 Hz and $200-2800 \mathrm{~Hz}$, respectively).

\begin{tabular}{|c|c|c|c|c|c|c|c|c|c|c|c|c|}
\hline & \multicolumn{2}{|c|}{ Mooring DMON SPL } & \multicolumn{2}{|c|}{ Transect DMON SPL } & \multicolumn{2}{|c|}{ Transect M20 SPL } & \multicolumn{2}{|c|}{ M20 PV X } & \multicolumn{2}{|c|}{ M20 PV Y } & \multicolumn{2}{|c|}{ M20 PV Z } \\
\hline Position & Dawn & Morning & Dawn & Morning & Dawn & Morning & Dawn & Morning & Dawn & Morning & Dawn & Morning \\
\hline Reef & 107.6 & 103.7 & 106.8 & 104.1 & 122.0 & 117.3 & -129.9 & -129.6 & -129.1 & -128.7 & -128.9 & -127.8 \\
\hline $50 \mathrm{~m}$ & 108.6 & 104.2 & 104.1 & 102.5 & 115.8 & 113.5 & -130.3 & -130.1 & -130.4 & -129.8 & -127.5 & -128.1 \\
\hline $150 \mathrm{~m}$ & 108.9 & 102.6 & 102.4 & 101.4 & 115.4 & 114.3 & -127.6 & -125.2 & -128.4 & -130.4 & -120.5 & -125.8 \\
\hline $500 \mathrm{~m}$ & 108.9 & 104.0 & 101.2 & . & 116.0 & - & -130.8 & - & -131.0 & - & -129.2 & - \\
\hline $1000 \mathrm{~m}$ & 108.1 & 102.6 & 101.0 & 103.0 & 115.7 & 112.7 & -130.5 & -129.8 & -131.0 & -131.2 & -129.1 & -128.3 \\
\hline $1500 \mathrm{~m}$ & 107.6 & 102.5 & 100.1 & 101.7 & 113.0 & 113.5 & -131.4 & -130.5 & -130.7 & -129.9 & -128.5 & -127.0 \\
\hline $500 \mathrm{~S} \mathrm{~m}$ & 104.6 & 103.1 & 101.0 & 102.8 & 114.5 & 113.7 & -131.3 & -130.6 & -131.6 & -130.4 & -130.2 & -129.5 \\
\hline $500 \mathrm{~N} \mathrm{~m}$ & 105.0 & 103.6 & 101.7 & 104.3 & 116.3 & 111.7 & -130.0 & -133.9 & -131.5 & -134.1 & -126.0 & -126.5 \\
\hline
\end{tabular}

Table 4.3: Exponential decay equations of the curve fit to full band sound pressure level (in $\mathrm{dB}$ re $1 \mu \mathrm{Pa}$ ) and particle velocities (in dB re $1 \mathrm{~m} \mathrm{~s}^{-1}$ ) displayed in Figures $4.3 \mathrm{c}, \mathrm{d}$ and $4.4 \mathrm{a}, \mathrm{b}$ respectively, where $\mathrm{y}$ is the sound level at a given distance and $\mathrm{x}$ is the distance from the reef.

\begin{tabular}{|c|c|c|c|c|c|c|c|c|c|}
\hline Transect & $\mathrm{SPL}_{r m s}$ & SPL $r^{2}$ & $\begin{array}{l}\text { SPL } \\
\text { p-value }\end{array}$ & XY Velocity & $\mathrm{XY} \mathrm{r}^{2}$ & $\begin{array}{l}\text { XY } \\
\text { p-value }\end{array}$ & Z Velocity & $\mathrm{Z} \mathrm{r}^{2}$ & $\begin{array}{l}Z \\
\text { p-value }\end{array}$ \\
\hline & $\begin{array}{l}y=107.1 * x^{-0.0086} \\
y=103.6 * x^{-0.0015}\end{array}$ & 0.105 & $\begin{array}{l}0.001 \\
0.5\end{array}$ & $\begin{array}{l}y=-127.6 * x^{0.0003} \\
y=-127 * x^{0.00023}\end{array}$ & 0.051 & $\begin{array}{l}0.02 \\
0.51\end{array}$ & $\begin{array}{l}y=-128.5 * x^{-0.00007} \\
y=-127.6 * x^{-0.00005}\end{array}$ & $\begin{array}{l}0.011 \\
0.006\end{array}$ & 0.86 \\
\hline
\end{tabular}




\subsection{Discussion}

A range of studies have demonstrated that larval fish and invertebrates may orient towards reef sound and perhaps even discern among sound types; however, there are very limited data on the distances over which acoustic cues might be used. Furthermore, there have been few direct measurements of acoustic particle motion, the relevant acoustic stimulus for most larval fishes and invertebrates, thus leaving this potential settlement cue overlooked. The current investigation represents an effort to fill this knowledge gap by quantifying this particle motion cue, particularly with respect to the distances over which reef sound propagates.

Reef sound pressure did attenuate with distance from the reef, but this varied both by frequency and time of day, likely as a result of variation in sound production. Attenuation was most apparent at dawn in both frequency bands and during the mid-morning in the high-frequency band only. Notably, the dip in sound level for the on-reef recorder (at the $400 \mathrm{~m}$ mark) during the dawn transect (Figure 4.3b) may reflect some of this diel periodicity as this was a parallel transect point and thus the last (latest) taken during each recording session. Coral reef soundscapes have a strong temporal periodicity (Staaterman et al., 2014; Kaplan et al., 2015). Given that sound production on many reefs including Hawaiian reefs has been shown to peak at dusk and dawn (Au et al., 2012), it is possible that these times of day represent a reliably higher sound level that larvae may use to orient. However, whether and how changing sound levels over time might affect larval sensing has yet to be determined.

The lack of agreement with cylindrical or spherical spreading predictions has been demonstrated on temperate reefs and has been postulated to be a result of a "reef effect" (Radford et al., 2011b), whereby levels do not attenuate as quickly as predicted because the reef is not a single point source but instead is composed of a "chorus" of many sound sources (fish, snapping shrimp). However, aural and visual inspections of our coral reef recordings collected far from the reef indicated no discrete detectable sounds of biological origin, which suggests that at greater distances from the reef such sounds might be buried in the environmental noise floor. Alternatively, the temperate reefs, which are acoustically dominated by feeding urchins (Radford et al., 2011b), may simply exhibit a different propagation pattern from coral reefs rich in fish and snapping shrimp sounds. In addition, propagation may be largely dependent on bathymetry and substrate (Urick, 1983). 


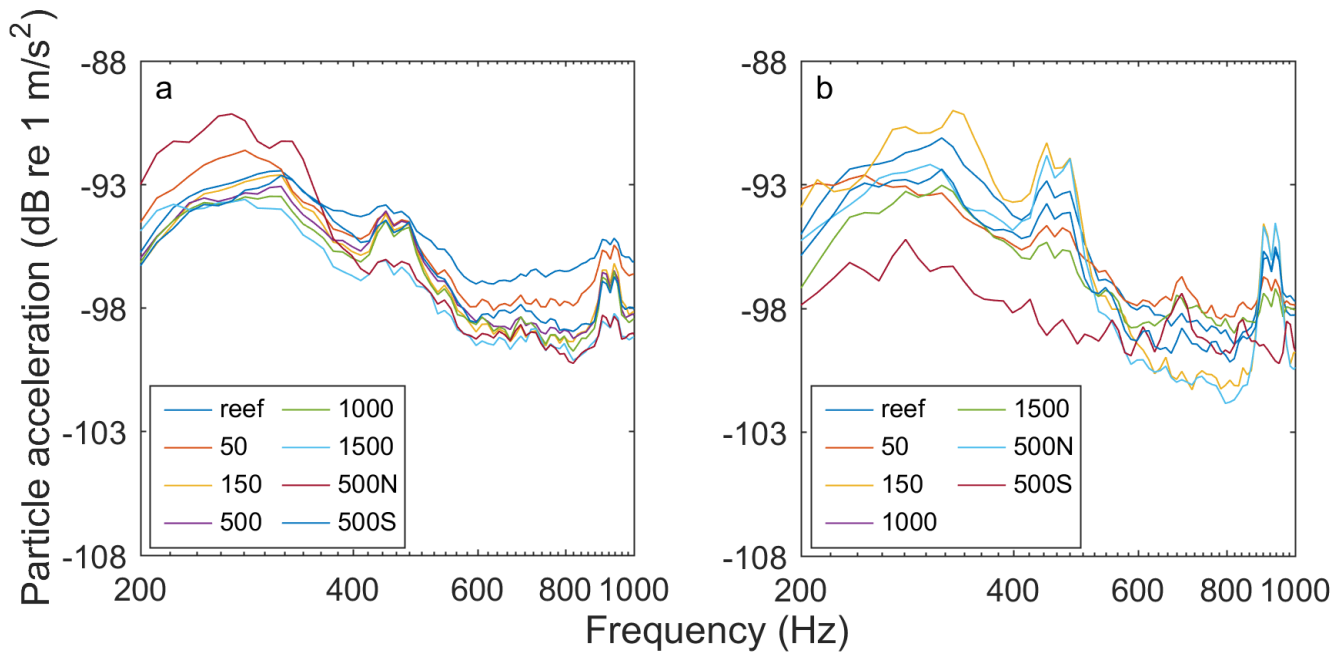

Figure 4.5: Mean particle acceleration values by transect, position, and frequency in the $\mathrm{x}$-axis. Peaks in acceleration were seen below $500 \mathrm{~Hz}$ in both transects, but no clear pattern with distance was evident. Acceleration was elevated slightly at the $500 \mathrm{~N}$ position during the dawn transect (a) and at the $150 \mathrm{~m}$ position during the mid-morning transect (b). No data were available for the $500 \mathrm{~m}$ position during the mid-morning transect.

Earlier work stressed the importance of identifying which coral reef soundscape components are the vital settlement cues: lower-frequency fish sounds or higher-frequency shrimp sounds (Simpson et al., 2008). The data presented here show that reef sound at high frequencies attenuates rapidly and that the particle acceleration and pressure levels in this band are relatively low. This suggests that it is unlikely that the high-frequency shrimp cues could be detected at any appreciable distance from the reef.

Here we show that the lower-frequency fish sounds were both higher in amplitude and propagated farther, particularly during crepuscular chorus events. In addition, there was a much larger number of high-amplitude transients in the low-frequency band. Furthermore, this frequency band overlaps the predominant hearing range of many of the marine fish and invertebrates that have been tested (Ladich and Fay, 2013; Mooney et al., 2010), suggesting that both the low-frequency chorus and/or these transients may offer viable cues for settlement. While sound levels appeared to be somewhat elevated below $200 \mathrm{~Hz}$ compared to levels between $200-1000 \mathrm{~Hz}$, the former frequency range was removed by filtering to ensure that the reported levels were predominately a result of biological sound production.

Particle velocity data collected on the M20 particle velocimeter corresponded somewhat to sound pressure data collected on the DMON acoustic recorder in terms of attenuation 
with distance, but there was considerable variability. The $\mathrm{z}$ axis showed velocities that were roughly as high as in the $\mathrm{x}-\mathrm{y}$ axes. The reason for this is not obvious; while it may be a result of the influence of non-acoustic sources (i.e., up-down motion from surface waves on the mooring system) despite the multiple absorption methods, physical movements of the mooring system are expected to be much lower frequency than the analysis bandwidths used here.

The acceleration data did not display any clear trends with distance from the reef in either transect. Furthermore, the position with the highest acceleration values was different in the dawn transect $(500 \mathrm{~N})$ than in the mid-morning transect $(150 \mathrm{~m})$. This differs from the velocity data (which do show a trend with distance). This is perhaps due to the fact that the acceleration data are frequency-specific; as a result, these data are more variable than the velocity data to which they are proportional.

Given that many fishes are likely able to sense particle motion and that it is the primary hearing modality for larval fishes (Mann et al., 2007) it is instructive to compare the particle acceleration values measured here to hearing thresholds. Radford et al. (2012) measured particle acceleration-derived hearing thresholds using a shaker table for the goldfish (Carassius auratus), bigeye (Pempheris adspersa), and common triplefin (Forstergyian lappillum). The best hearing sensitivity for all 3 species was between $100-200 \mathrm{~Hz}$ and was approximately $-60 \mathrm{~dB}$ re $1 \mathrm{~m} \mathrm{~s}^{-2}$, which is approximately $25 \mathrm{~dB}$ higher than the highest acceleration values measured at any position in this study, indicating that those species probably could not hear the average Olowalu reef signal at any of these distances. Conversely, Horodysky et al. (2008) measured the hearing thresholds using a speaker in air for 6 sciaenid species and found considerably lower particle acceleration thresholds (approximately $-100 \mathrm{~dB}$ re $1 \mathrm{~m} \mathrm{~s}^{-2}$ for the Atlantic croaker (Micropogonias undulatus) at $100 \mathrm{~Hz}$ ), which suggests that some fishes could detect this reef at the distances measured. While the hearing abilities of larval and the majority of adult invertebrates have yet to be measured, Mooney et al. (2010) measured the acceleration-based hearing thresholds of adult longfin squid (Doryteuthis pealeii) and found that their best thresholds were $-35 \mathrm{~dB}$ re $1 \mathrm{~m} \mathrm{~s}^{-2}$ at $200 \mathrm{~Hz}$, indicating that these squid could not hear this reef at any distance. Similarly, Packard et al. (1990) tested the low-frequency hearing of cuttlefish (Sepia officinalis), squid (Loligo vulgaris), and octopus (Octopus vulgaris) and identified a threshold level of -40 dB re $1 \mathrm{~m} \mathrm{~s}^{-2}$ at $10 \mathrm{~Hz}$. Finally, Roberts et al. (2015) tested the response to vibration of the 
blue mussel (Mytilus edulis) and found a best sensitivity of $-22 \mathrm{~dB}$ re $1 \mathrm{~m} \mathrm{~s}^{-2}$ at $10 \mathrm{~Hz}$. Because reef sound is broadband and these hearing thresholds are derived using pure tones, it is prudent to increase the audiogram sensitivity by $10 \log (0.1 \times F)$, where $\mathrm{F}$ is the tone frequency, to account for the critical bandwidth (Egner and Mann, 2005). However, doing so does not change which of these species could theoretically detect the reef based on the available data.

The majority of these hearing studies did not report background noise levels (but see Roberts et al., 2015). The amount that individual signals are elevated with respect to the background noise is critical to determining both whether a larva could detect the signals and the potential for masking. For example, a study on the hearing abilities of goldfish (C. auratus), catfish (Platydoras costatus), and sunfish (Lepomis gibbosus) found that in the presence of masking noise, hearing thresholds are considerably elevated (Wysocki and Ladich, 2005).

The reef sound levels reported here are averages over a minute of recording. Thus, there will be variability above and below the mean, with some sounds exceeding the average levels. Whether those sounds are detectable will depend on their amplitude, spectral composition, the amount by which they exceed the background noise, and the hearing abilities of a given species. Indeed, when the SPL of short $(5 \mathrm{~ms})$ segments was pooled by recording position across the recording days and transect times, there were a considerable number of high-amplitude transient signals, some of which were $20 \mathrm{~dB}$ (i.e., an order of magnitude) above median levels (Figure 4.6). Many more of these transients were found in the lowfrequency band, where larval hearing is likely more sensitive, than in the high-frequency band. Accordingly, while larval animals might not be able to hear the average sound level at any appreciable distance from the reef, they might detect some of these transient signals. Indeed, it is possible that an individual high-amplitude signal that originated on the reef might propagate according to theoretical predictions; however, detection of such cues was not possible in the present study design.

In conclusion, coral reef sound pressure levels attenuated with distance from the reef but not to the extent predicted by theory, perhaps as a result of the ambient noise levels of the area and the relatively shallow water (less than $100 \mathrm{~m}$ ) off the reef. Attenuation with distance from the reef was more evident during dawn, when crepuscular biological activity is greatest. By mid-morning, such a relationship between sound level and distance 

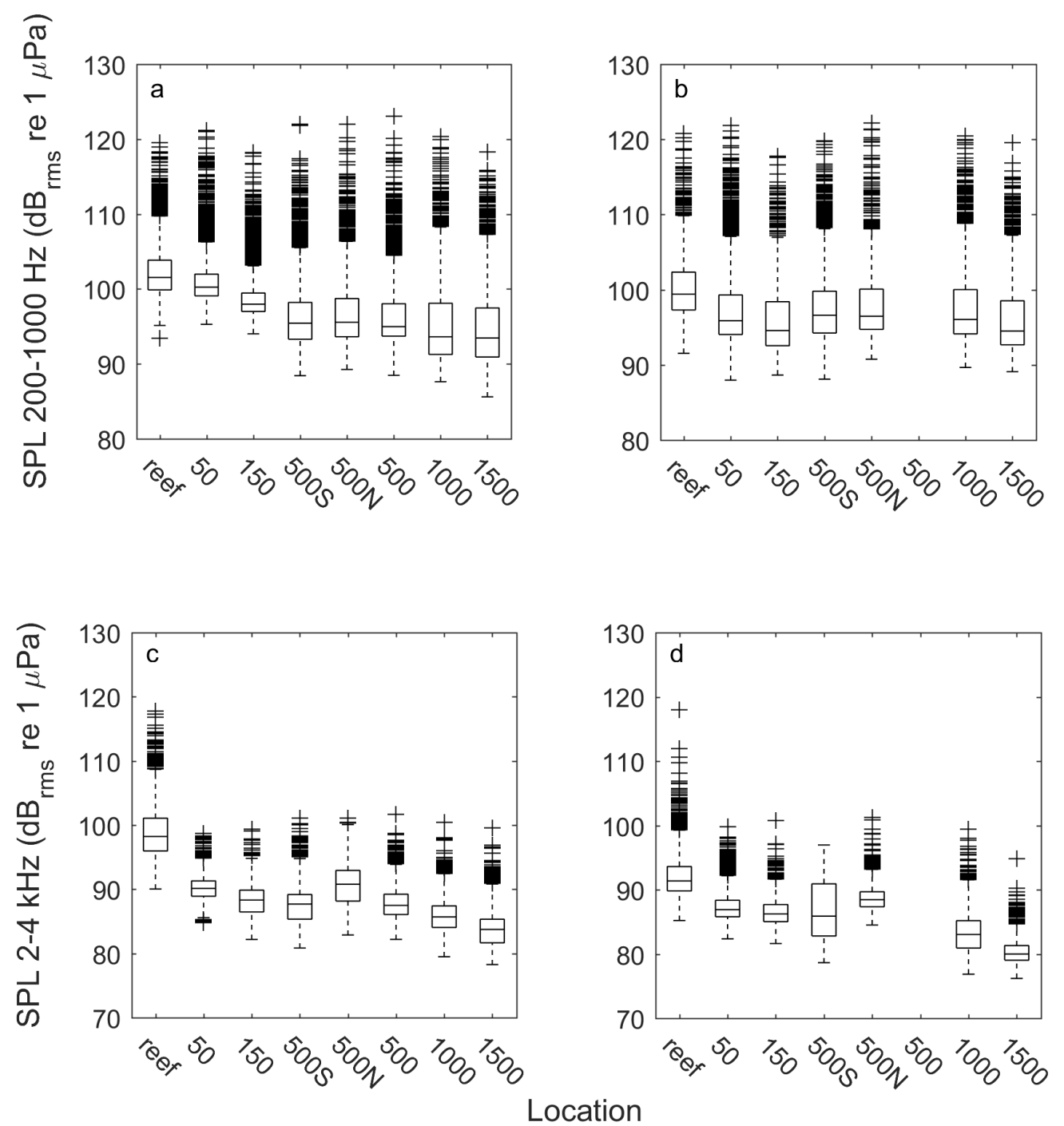

Figure 4.6: Boxplots showing SPL in $5 \mathrm{~ms}$ windows in low- $(200-1000 \mathrm{~Hz})$ and highfrequency $(2000-4000 \mathrm{~Hz}$ ) bands (a-d respectively) during the dawn (a,c) and mid-morning $(\mathrm{b}, \mathrm{d})$ transects pooled across sampling days. While the median SPL values roughly follow the same trend as that for the mean values displayed in Figure 4.3, there is considerable spread (in particular in the low-frequency band) and there are multiple high-amplitude outliers for nearly every recording position. The number of high-amplitude outliers relative to the lower amplitude averages suggests that while larval animals may not be able to hear the average sound from a reef, it is possible that they could detect some high-amplitude transients. No data were available for the $500 \mathrm{~m}$ position during the mid-morning transect. Central bar median; box - 25-75 percentiles; whiskers - most extreme data points not considered as outliers; crosses - outliers.

was not apparent. Similar trends were apparent for particle velocity but with considerable variability, and no such trend was apparent for particle acceleration. Because there was no 
particle velocity sensor on the reef for the duration of each transect, it is not possible to state unequivocally that on-reef particle velocity levels were relatively constant over the course of the transect, even though within-transect temporal variability in sound pressure levels was limited. Average particle acceleration levels were at or below published hearing thresholds, which suggests that these sounds may be perhaps too faint to be used as an orientation cue for marine larvae in search of suitable settlement habitat, at least at distances beyond 150 $\mathrm{m}$. However, the high-amplitude transient signals present at nearly every distance from the reef and on both transects might be detectable at greater ranges. Additionally, if other reefs or other times of day exceeded the mean values presented here, those might be detectable, depending on their attributes and the hearing abilities of a given larva.

These data show that the temporal and spatial scales of the available acoustic cues vary, yet seemingly do not offer a long-distance (several $\mathrm{km}$ ) cue. However, the actual scales over which acoustic cues may function, at particular times and places, remain unresolved. If larvae are not using sound as a long-distance orientation cue but are responding directionally to sound, perhaps acoustic cues could offer a shorter range cue for settling animals; for example, once they are close to a reef. As many larvae approach reefs at night (when vision is presumably limited), sound cues might offer an indication that a larva is above that reef and could then successfully settle. More comprehensive measurements at key times (i.e., night) would be valuable to assess the scales over which reef sounds may play a role in larval processes. Additionally, acoustic signals are now known to operate as a contact call for some fishes (Radford et al., 2015). Sound cues might thus reflect finer-scale assessments of a reef such as habitat of nearby soniferous predators or conspecifics to respectively avoid or approach. 


\section{Chapter 5}

\section{Ambient noise and temporal patterns}

of boat activity in the U.S. Virgin

Islands National Park

This chapter was originally published as: Kaplan, M.B. \& Mooney, T.A. 2015. Ambient noise and temporal patterns of boat activity in the U.S. Virgin Islands National Park. Marine Pollution Bulletin 98(2015):221-228.DOI: $10.1016 /$ j.marpolbul.2015.06.047. Used with permission as granted in the original copyright agreement. 


\subsection{Abstract}

Human activity contributes noise to marine ecosystems. Recent studies have examined the effects of boat noise on marine fishes, but there is limited understanding of the prevalence of this type of sound source. This investigation tracks vessel noise on 3 reefs in the U.S. Virgin Islands National Park over 4 months in 2013. Ambient noise levels ranged from 106 to $129 \mathrm{~dB}_{\mathrm{rms}}$ re $1 \mathrm{\mu Pa}(100 \mathrm{~Hz}-20 \mathrm{kHz})$. Boat noise occurred in $6-12 \%$ of samples. In the presence of boat noise, ambient noise in a low-frequency band $(100-1000 \mathrm{~Hz})$ increased by $>7 \mathrm{~dB}$ above baseline levels and sound levels were significantly higher. The frequency with the most acoustic energy shifted to a significantly lower frequency when boat noise was present during the day. These results indicate the abundance of boat noise and its overlap with reef organism sound production, raising concern for the communication abilities of these animals.

\subsection{Introduction}

Anthropogenic noise is increasingly prevalent in the global ocean (reviewed in Hildebrand, 2009). Human activities such as shipping, pile driving, geophysical exploration, and sonar all introduce noise into the marine environment and this noise can propagate over a range of spatial scales (Urick, 1984). Anthropogenic noise may affect the behavior and physiology of marine organisms from invertebrates (Beets and Friedlander, 1998; Pine et al., 2012) to fishes (Popper and Hastings, 2009) and marine mammals (Di Iorio and Clark, 2010). However, noise levels and their effects are largely unknown (Slabbekoorn et al., 2010).

Much of the documented increase in ocean noise levels has been attributed to commercial shipping activities (Andrew et al., 2011; Chapman and Price, 2011; McDonald et al., 2006) and has primarily been quantified for open-ocean environments. However, small boats can act as transient, high-amplitude noise sources (e.g., Erbe, 2002). These vessels are often operated in near-shore, coastal waters and overlap spatially with a range of ecosystems (e.g., Codarin et al., 2009). At present, the extent to and timescales over which small vessel traffic increases ambient noise levels are unknown for most habitats.

As ocean noise increases, so does concern for its impacts on the behavior and physiology of marine animals. Effects have been documented from both transient and continuous anthropogenic noise, with research largely focusing on high-amplitude sources such as air 
guns (e.g., Fewtrell and McCauley, 2012; McCauley et al., 2003; Popper et al., 2005). However, there is growing evidence that small boat noise can impact fishes. Exposure to boat noise from a range of vessels disrupted schooling behavior in captive bluefin tuna, which the authors argued could affect feeding if a similar response occurred in wild tuna (Sara et al., 2007). Playbacks of vessel noise in the lab raised hearing thresholds for 3 species of Mediterranean fish, particularly in the frequency range where acoustic communication takes place (Codarin et al., 2009). There is some evidence that boat noise may disrupt orientation behavior in captive larval fish (Holles et al., 2013); however, the extent to which this may occur in the wild is unknown.

Vessel sounds may also help quantify how often boats enter areas of interest. While commercial ship activity can be tracked via Automatic Identification System (AIS) software (Hatch et al., 2008), this technology is typically not used aboard smaller boats. However, small boat presence can be tracked through vessel engine noise (Lammers et al., 2008). Listening for this noise may offer resource managers a way to track the occurrence of at least some boats. Such a tool may be particularly valuable in marine protected areas or locations that are not easily accessed or monitored visually.

In light of these data limitations on small boat noise prevalence and characteristics in coastal waters, and the potential utility of boat noise as a means of tracking small vessel activity, the purpose of this investigation was to characterize the diel, weekly and summer trends in boat noise at 3 coral reefs located off the island of St. John in the U.S. Virgin Islands National Park. St. John contains a popular marine park, seeing ca. 500,000 visitors per year, many of whom use boats to access local reefs. The island is nearly $60 \%$ National Park, with the Park containing ca. 5650 acres of submerged coral reefs, mangrove, and seagrass habitats. It is also a system under stress, seeing declines in coral cover in recent years (Edmunds, 2013). The quantification of potential stressors such as boat noise is needed

to gauge the extent of human activity in this ecosystem. The results present a means to potentially track boat occurrence and noise levels in areas of interest.

\subsection{Methods}

Three reefs located in the U.S. Virgin Islands National Park were instrumented with acoustic recording devices for ca. 4 months, starting in April 2013 (Figure 5.1). Reefs were chosen 
based on long-term survey data (Edmunds, 2013) and a rapid, preliminary visual survey of 10 reefs in the area. Two of these - Tektite and Yawzi Point - have been studied for 25 years (see Edmunds, 2013, for review). The third reef - Ram Head - was selected as a comparison site. Mooring balls were located near each of these reefs, some of which were for daytime use only while others could be used for overnight mooring. Tektite ranged from $9-18 \mathrm{~m}$ depth and consisted of a large sloping reef face, Yawzi ranged from ca. 5-10 m depth and was composed of a large mound that sloped down to sand, and Ram Head ranged from ca. 8-13 $\mathrm{m}$ and was mostly flat, with patch reef sparsely located throughout the site. All 3 reefs were similar in distance from shore and wave exposure (Figure 5.1).

Recordings were collected using 2 types of autonomous underwater recording devices: the DMON (Woods Hole Oceanographic Institution, Woods Hole, MA) and the DSG (Loggerhead Instruments, Sarasota, FL). The DMONs were configured with a low-noise preamplifier (20 dB gain), $13.2 \mathrm{~dB}$ user programmable gain, a 6-pole Sallen-Key anti-alias filter, a 16-bit analog-to-digital converter, and 32 GB of FLASH memory. We programmed the DMON to record on 2 hydrophone (Navy type II ceramics) channels: LF (16 kHz sample rate with an anti-aliasing filter at $7.5 \mathrm{kHz}$ and high pass filter at $8 \mathrm{~Hz})$ and MF $(120 \mathrm{kHz}$ sample rate with an anti-aliasing filter at $50 \mathrm{kHz}$ and high pass filter at $100 \mathrm{~Hz}$ ). The DSG records on a single channel at $80 \mathrm{kHz}$ sample rate using a HTI-96 hydrophone (High-Tech Inc., Gulfport, Mississippi) and contains a 16-bit computer board. There is a user-selectable gain setting; for these recordings, $20 \mathrm{~dB}$ was used, which results in a high-pass filter being implemented at $80 \mathrm{~Hz}$. Two concrete moorings (ca. $100 \mathrm{lbs}$ in air) were prepared for each reef. Mooring 1 consisted of a DMON with customized duty-cycling software $(2.5 \mathrm{~min} / 2 \mathrm{~h}$, $2 \%$ duty-cycle) and a DSG acoustic recorder (1 $\mathrm{min} / 20 \mathrm{~min}, 5 \%$ duty cycle). Mooring 2 consisted of a DMON only. Acoustic recorders were attached to the mooring horizontally using hose clamps and cable ties, and hydrophones were ca. $0.3 \mathrm{~m}$ off the bottom. Moorings were deployed by SCUBA between 17-19 April 2013 and retrieved between 2-3 August 2013, yielding approximately 103 days of potential data collection per site.

The redundancy of recorders proved essential as the DSGs deployed at Yawzi and Ram Head did not successfully record, and the only instrument to properly record at Tektite was the DSG. As a result, acoustic comparisons between sites involved multiple recording devices. Only the first $60 \mathrm{~s}$ of the $2.5 \mathrm{~min}$ DMON recordings were used, and 1 minute from every 2 hours was taken from the DSG recordings such that there was temporal overlap 


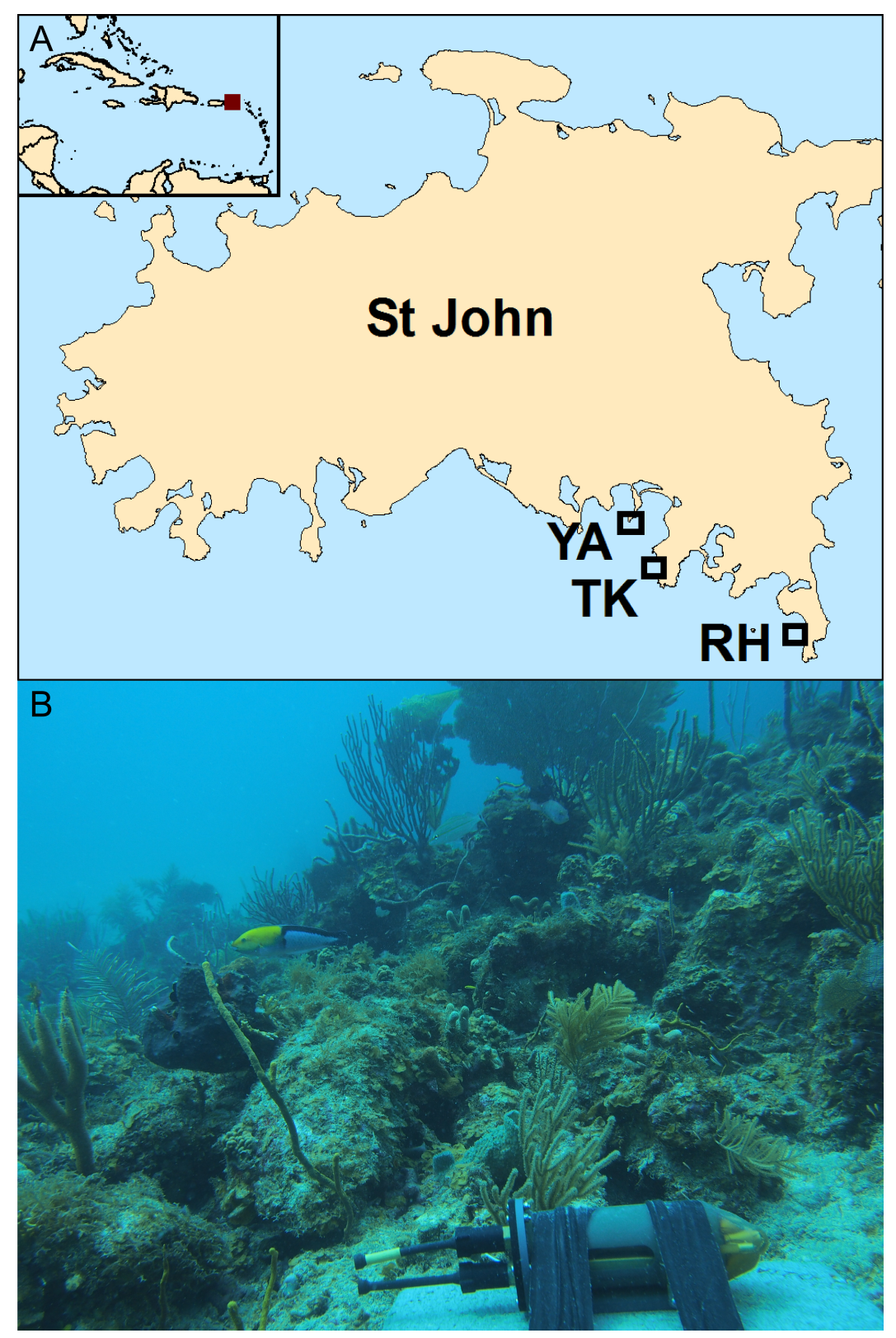

Figure 5.1: Deployment map (A) showing locations of 3 reefs located within the U.S. Virgin Islands National Park on which acoustic recording devices were deployed (TK-Tektite, YAYawzi, RH-Ram Head) in 2013. Example of an acoustic recorder mooring (B) showing a DMON (arrow points to hydrophones).

across reefs. The recording durations were as follows: Tektite - 19 April—6 July 2013; Yawzi - 17 April-1 August 2013; Ram Head - 19 April—2 August 2013.

Boat noise and any other sporadic noise was identified visually and confirmed aurally using long-term spectral average (LTSA) plots created in Triton (version 1.90; Scripps Whale Acoustics Lab, San Diego, CA). The LTSAs were computed with 2 s averages and in 200 
$\mathrm{Hz}$ bins. Boat noise events were summed by hour of day, week and month to describe the temporal distribution across the sampled periods.

All acoustic analyses were carried out in Matlab 8.1 (The MathWorks, Inc., Natick, MA). Analyzed files were corrected for calibrated hydrophone sensitivity and resampled to $44 \mathrm{kHz}$ because frequencies higher than $22 \mathrm{kHz}$ were not of interest for this study. Spectral analysis used Fast Fourier Transform (FFT) size of 880 points with a Hamming window and no overlap, which yielded a spectral resolution of $50 \mathrm{~Hz}$ and a temporal resolution of $20 \mathrm{~ms}$.

Each 60-s sound file was band pass filtered $(100-20,500 \mathrm{~Hz})$, and median peak frequency (the frequency with the highest power) and percentiles were calculated for the day (06:0018:00) and night (20:00 - 04:00) periods. The median was used because of the wide range in peak frequencies and the potential for outliers to bias estimates. Median sound pressure level (SPL) and percentiles in root-mean-square $\left(\mathrm{dB}_{\mathrm{rms}}\right)$ were calculated separately for 3 frequency bands - a low-frequency fish band (100-1000 Hz), a high-frequency snapping shrimp band $(2-20 \mathrm{kHz})$ and the full bandwidth $(100-20,000 \mathrm{~Hz})$.

Medians were compared statistically using Kruskal-Wallis tests and the critical p-value was corrected for multiple comparisons using the Bonferroni correction.

\subsection{Results}

Boats were detected at all 3 reefs throughout the deployment period (Figure 5.2C). Tektite had the highest number of detections, followed by Ram Head and Yawzi (Table 5.1). Consequently, the Tektite deployment had the highest proportion of recordings that contained boat noise. In addition, approximately $25 \%$ of deployment days were free of vessel noise at Tektite, whereas roughly half of the deployment days were free of vessel noise at Yawzi and Ram Head. Similarly, Tektite was exposed to the highest proportion of boat noise nearly every day of the week (Figure 5.2B). There were no significant differences in boat presence by day of week (Tektite: $\chi_{6}^{2}=0.059 ; \mathrm{p}>0: 05$; Yawzi: $\chi_{6}^{2}=0.066 ; \mathrm{p}>0: 05$; Ram Head: $\chi_{6}^{2}=0.063 ; \mathrm{p}>0: 05 ;$ Figure 5.2). However, there was a clear diel trend, with significant differences in boat presence by time of day on all reefs (Tektite: $\chi_{11}^{2}=98.2 ; \mathrm{p}<0: 0001$; Yawzi: $\chi_{11}^{2}=54.3 ; \mathrm{p}<0$ 0:0001; Ram Head: $\chi_{11}^{2}=41.5 ; \mathrm{p}<0$ 0:0001 Figure 5.2A). At Tektite, the hours of 08:00, 20:00 and 22:00 showed the greatest proportions of boat noise. There was a substantial decrease in boat detections in the early morning hours (00:00-04:00) and 
a brief lull around 10:00-12:00 at all reefs.

Table 5.1: Boat noise occurrences and proportion of recording time with boat noise by reef.

\begin{tabular}{l|l|l|l|l} 
Reef & $\begin{array}{l}\text { Number of } \\
\text { boat noise } \\
\text { occurrences }\end{array}$ & $\begin{array}{l}\text { Total minutes } \\
\text { recorded }\end{array}$ & $\begin{array}{l}\text { Proportion of } \\
\text { minutes with } \\
\text { boat noise }\end{array}$ & $\begin{array}{l}\text { Proportion of } \\
\text { days free of } \\
\text { boat noise }\end{array}$ \\
\hline Tektite & 115 & 939 & 0.12 & 0.24 \\
Yawzi & 72 & 1267 & 0.06 & 0.48 \\
Ram Head & 83 & 1257 & 0.07 & 0.5
\end{tabular}

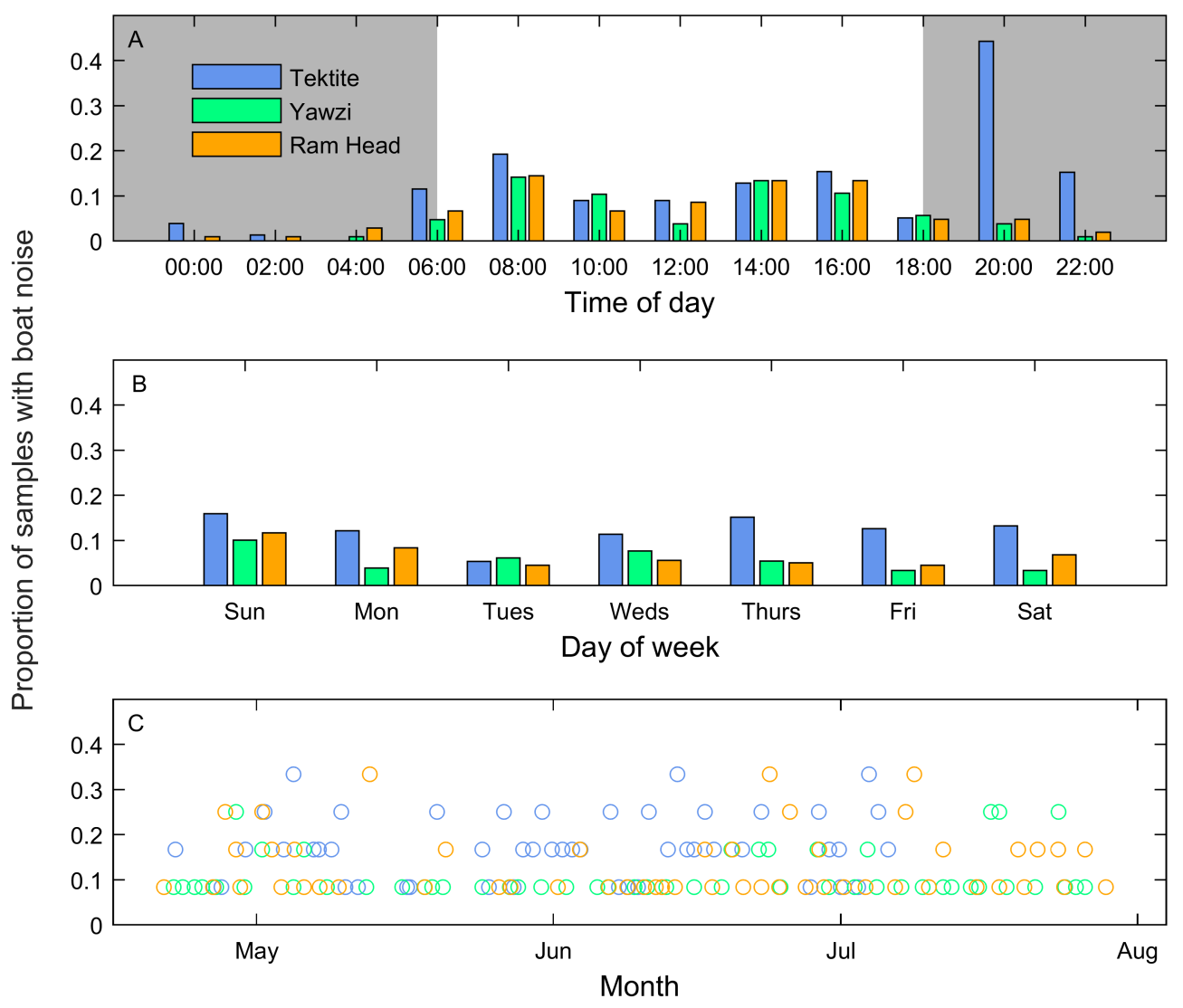

Figure 5.2: Summary of the presence of boat noise at 3 reefs in the U.S. Virgin Islands from April to August 2013 (A) by time of day (gray is 20:00-04:00), (B) by day of week, and (C) summed by day over the entire deployment period.

Ambient noise levels at the 3 reefs ranged from $88-130 \mathrm{~dB}$ in the low-frequency band $(100-1000 \mathrm{~Hz}), 106-126 \mathrm{~dB}$ in the high-frequency band $(2-20 \mathrm{kHz})$, and $106-129 \mathrm{~dB}$ in the full band (100-20000 Hz; Table 5.2). Power spectral density followed a roughly similar pattern at all 3 reefs, with elevated low frequency sound levels, a trough between $2-5 \mathrm{kHz}$ and elevated sound levels from $5-15 \mathrm{kHz}$ (Figure 5.3). 
Table 5.2: Sound pressure levels in 3 frequency bands at 3 reefs.

\begin{tabular}{l|l|l|l|l|l|l|l|l|l} 
& \multicolumn{8}{|c}{ SPL $\left(\mathrm{dB}_{r m s}\right.$ re $\left.1 \mu \mathrm{Pa}\right)$} \\
\hline & \multicolumn{3}{|c}{$100-1000 \mathrm{~Hz}$} & \multicolumn{3}{c|}{$2-20 \mathrm{kHz}$} & \multicolumn{3}{c}{$100-20000 \mathrm{~Hz}$} \\
\hline Reef & Min & Median & Max & Min & Median & Max & Min & Median & Max \\
\hline Tektite & 98.1 & 103.3 & 130.6 & 106.5 & 109.6 & 121.8 & 106.6 & 110 & 126.1 \\
Yawzi & 89.8 & 93.8 & 125.3 & 110.2 & 113.2 & 126.4 & 110.3 & 113.3 & 129.8 \\
Ram Head & 88.4 & 92.4 & 124.2 & 108.6 & 111.1 & 124.8 & 108.8 & 111.4 & 128.4
\end{tabular}

There were notable differences in sound intensity between sound files that contained boat noise and those that did not for a given reef and at a given time of day. Median low-frequency SPL was always significantly higher in the presence of boat noise (Figure 5.4 and Table 5.3) and was elevated by up to $10 \mathrm{~dB}$ during the day and by up to $7 \mathrm{~dB}$ at night compared to sound files without boat noise.

There were also spectral differences among sound files based on the presence of boat noise. Median peak frequency was significantly lower in the presence of boat noise during the day but not at night for all 3 reefs (Figure 5.5 and Table 5.3).

Sound files with boat noise present had considerably greater low-frequency energy content at frequencies below $1000 \mathrm{~Hz}$, where power spectral density could be $20 \mathrm{~dB}$ greater at certain frequencies (Figure 5.6). There was some variation among incidences of boat noise but the associated power spectra were broadly similar (Figure 5.7). Peak frequencies were typically between 100 and $500 \mathrm{~Hz}$ when vessel noise was present.

\subsection{Discussion}

Anthropogenic noise is increasing in many parts of the oceans, yet the extent to which various acoustic frequencies and sound levels are changing is often uncertain, particularly in dynamic, coastal ecosystems. Because increased noise may affect the behavior and physiology of various marine organisms, detailed assessments of noise levels are needed for many habitats to better understand the extent to which animals may be exposed to increasing noise. The current effort represents an initial measure of small boat activity at 3 coral reefs in the U.S. Virgin Islands National Park. There was substantial overlap between vessel noise and the relevant frequency bands for fish communication and hearing. The abundance of boat noise on these reefs reflects the prevalence of this potential stressor. However, boat noise also stands out as an obvious cue to monitor the occurrence of human activity on these 

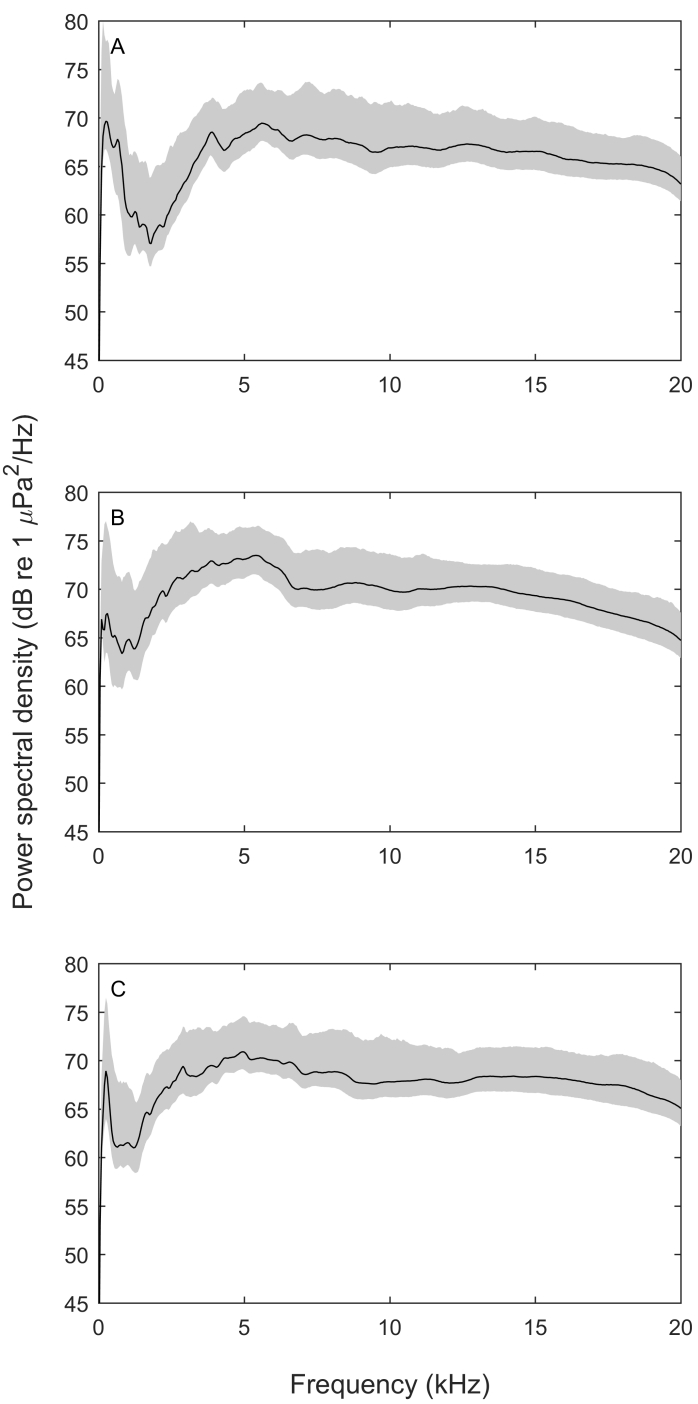

Figure 5.3: Background noise measured as full bandwidth $(10 \mathrm{~Hz}-20 \mathrm{kHz})$ for the full sampled period for (A) Tektite, (B) Yawzi and (C) Ram Head. Line is median with shaded area depicting $5-95$ percentiles.

reefs and other coastal ecosystems, where such data is urgently needed.

In general, the pattern of boat noise observations would seem to reflect human activity, with more noise when people are awake and active (daytime) and little activity in early morning hours. Peaks near 08:00 could reflect transiting to or from mooring balls nearby. There were no differences in boat activity by day of week, which suggests relatively consistent activity irrespective of day. While it is uncertain precisely why Tektite demonstrated 

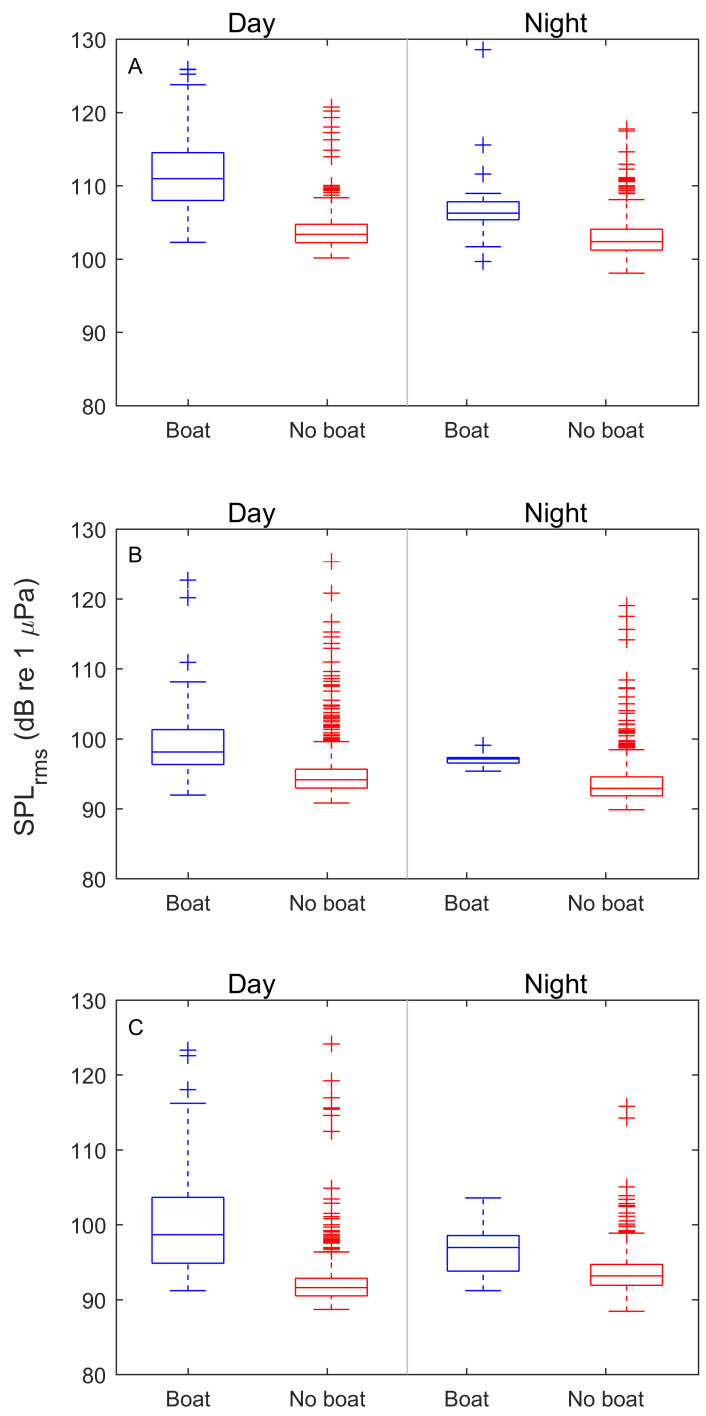

Figure 5.4: Low-frequency sound pressure level $(100-1000 \mathrm{~Hz})$ during times of day with boat noise present (blue) and otherwise (red) at each of 3 reefs in the U.S. Virgin Islands (A, Tektite; B, Yawzi; C, Ram Head). SPL was always significantly higher when boat noise was present during both day and night. Central bar - median; box - 25-75 percentiles; whiskers - most extreme data points not considered as outliers; crosses - outliers.

a substantial peak at 20:00, it may result from running engines or generators on nearby moorings.

Sound pressure levels pooled across reefs from the entire deployment period varied approximately $40 \mathrm{~dB}$ in the low-frequency band, whereas levels varied only about $20 \mathrm{~dB}$ in the high-frequency band. This is likely a result of the fact that vessel noise is pre- 

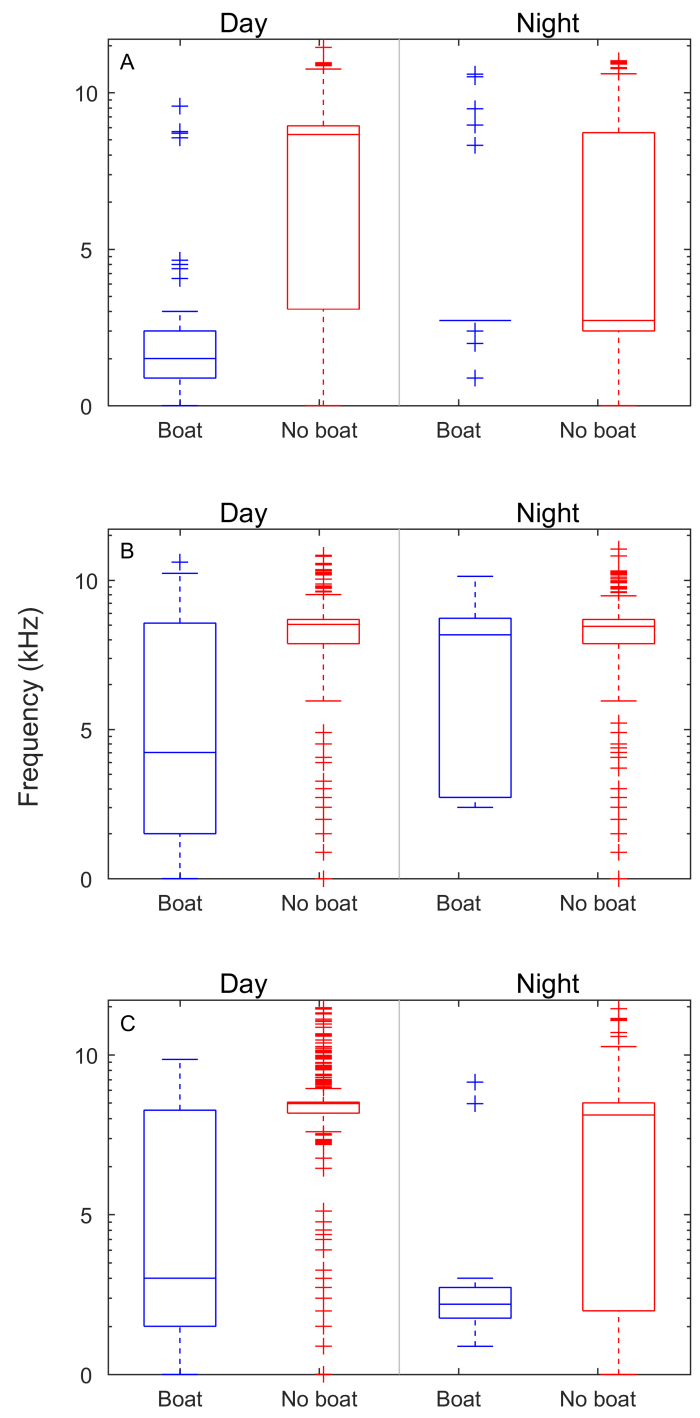

Figure 5.5: Peak frequency during times of day with boat noise (blue) and otherwise (red) at each of 3 reefs in the U.S. Virgin Islands (A, Tektite; B, Yawzi; C, Ram Head). Peak frequency was significantly lower when boat noise was present than otherwise for each reef during the day, but there were no significant differences in peak frequencies at night. Central bar - median; box - 25-75 percentiles; whiskers - most extreme data points not considered as outliers; crosses - outliers.

dominately low frequency and therefore has a disproportionate effect on sound levels and variability below about $5 \mathrm{kHz}$.

The shapes of power spectra from vessel-free recordings were consistent with coral reef soundscapes in which the dominant sound source is snapping shrimp (Cato and Bell, 1992), 

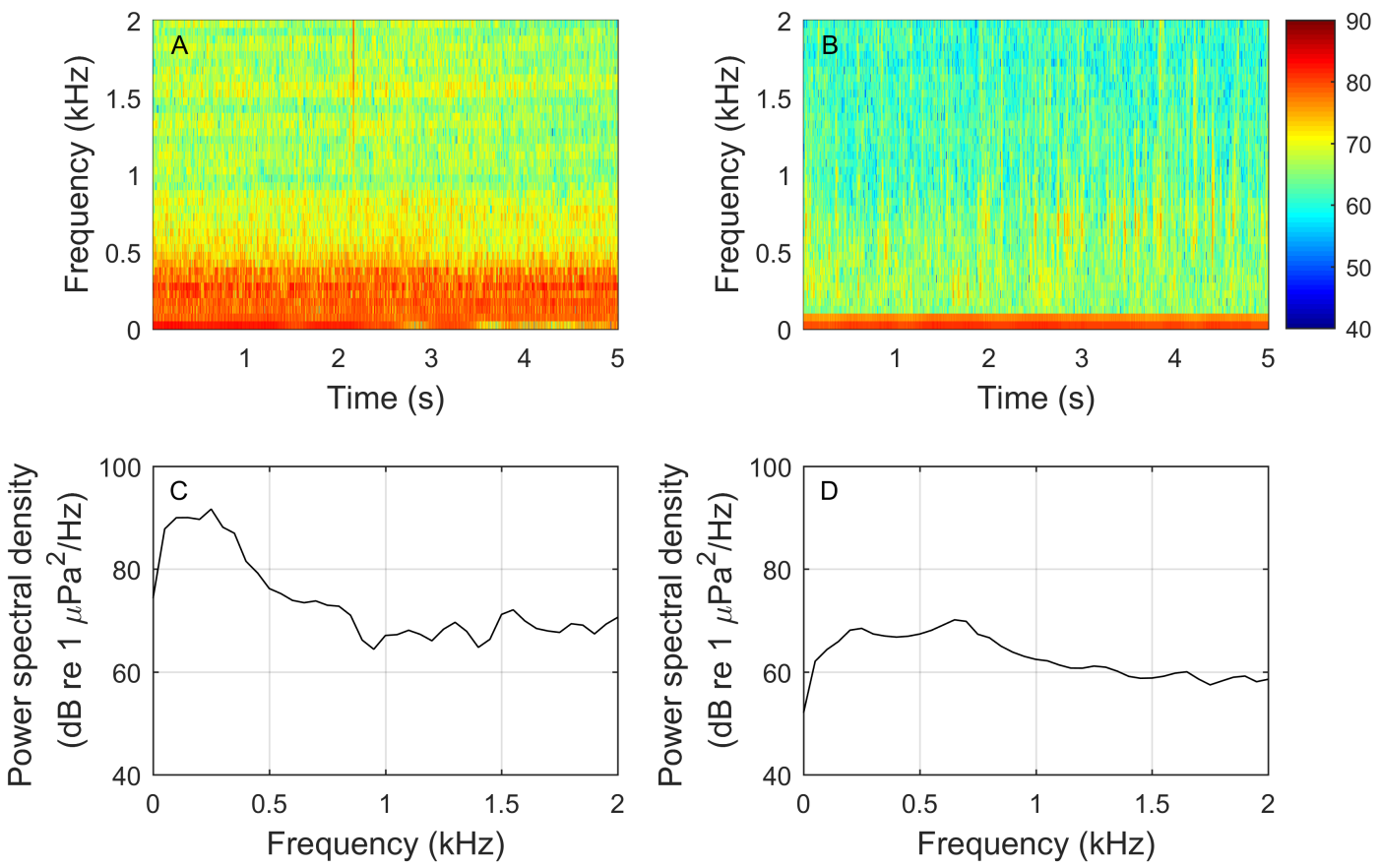

Figure 5.6: Spectrograms of the first 5 seconds of recordings made at 18:00 at Tektite on 2 consecutive days in June with boat noise present (A) and absent (B) and associated power spectra $(\mathrm{C}$ and $\mathrm{D})$. Color bar units are $\mathrm{dB}$ re $1 \mu \mathrm{Pa}$.

thus elevating acoustic energy at higher frequencies compared to open-ocean noise spectra (Hildebrand, 2009). However, differences between the shallow water spectra reported here and open ocean spectra could also result from differences in acoustic propagation.

Acoustic recordings made in the presence of boats had lower peak frequencies and higher sound pressure values. In the absence of boat noise, peak frequency was relatively high (ca. $5 \mathrm{kHz}$ ) as a result of snapping shrimp acoustic activity, which is ubiquitous in tropical, coastal habitats (Table 5.3). Lower peak frequencies at Tektite at night (when boats were detected less) could be a result of elevated fish calling activity at that site (Kaplan et al., 2015). The range of peak frequencies when boats are present could be a result of variability with respect to vessel engine types, speeds, and distances to the hydrophone. 
Table 5.3: Statistical comparison of sound pressure level and peak frequency between day and night and boat presence and absence for each reef. Medians shown with 25-75 percentiles.

\begin{tabular}{l|l|l|l|l|l|l|l}
\multicolumn{9}{|c}{ SPL 100-1000 Hz $\left(\mathrm{dB}_{r m s}\right.$ re 1 $\left.\mu \mathrm{Pa}\right)$} \\
\cline { 2 - 6 } & Reef & Time of Day & Boat Absent: Median $(25-75 \%)$ & Boat Present: Median $(25-75 \%)$ & $\chi^{2}$ & $\mathrm{df}$ & $\mathrm{p}$ \\
\hline \multirow{2}{*}{ Tektite } & Day & $103.4(102.2-104.7)$ & $111.0(108.0-114.5)$ & 132.8028 & 545 & $<0.0001$ \\
& Night & $102.4(101.2-104.1)$ & $106.3(105.4-107.8)$ & 62.755 & 390 & $<0.0001$ \\
\hline \multirow{2}{*}{ Yawzi } & Day & $94.1(92.9-95.6$ & $98.1(96.3-101.3)$ & $97.1(96.5-97.3)$ & 70.4118 & 736 & $<0.0001$ \\
& Night & $92.9(91.8-94.6)$ & $98.7(94.9-103.7)$ & 11.3823 & 527 & $<0.001$ \\
\hline \multirow{2}{*}{ Ram Head } & Day & $91.6(90.5-92.8)$ & 137.8874 & 728 & $<0.0001$ \\
& Night & $93.2(91.9-94.7)$ & $96.9(93.8-98.5)$ & 11.4715 & 522 & $<0.001$ \\
\hline
\end{tabular}


Fish sounds and hearing abilities (for species without auditory specializations) are largely below $1000 \mathrm{~Hz}$ (Popper and Fay, 2011; Tricas and Boyle, 2014). The frequency overlap with vessel noise could result in masking of sounds vital to reproduction, feeding and territorial defense (Ladich, 2013), adding another stressor on these already impacted reefs (Figure 5.8). While these sound levels were far below those which induce temporary hearing loss (Smith et al., 2004), they occurred frequently, suggesting that the exposure durations and overall energy of introduced noise might be relatively high. It has been suggested that boat noise may impact the behavior of larval fish settling on reefs (e.g., Holles et al., 2013). This might indicate that reefs exposed to boats such as these might see such impacts. This is a particular concern for reefs that are already declining in recruitment and coral or fish abundance. Understanding the extent and mechanism of these effects is in its infancy and more work is needed to characterize the effects of this masking noise on behavior, recruitment and resiliency of reefs.

The trends shown here suggest that soundscape recordings can be used to track human activity. While relationships between container ship speed and sound level have been identified (McKenna et al., 2013), such data are limited for smaller vessels. Thus, visual observations would help to assign noise signatures to vessel types and relate received levels to vessel speed. These measurements may be particularly valuable in marine protected areas such as this study site or in remote reefs where quantifying fishing or other human activity is needed.

Boat noise can be highly transient, varying in both space and time; accordingly, further investigations should use a duty cycle with higher temporal coverage in order to increase the probability that boats that do pass through a given area are detected. The development of automatic detection algorithms for boat noise have been hindered by the variable nature of this source (e.g., speed, engine size and type, direction of movement); however, using SPL as an aural and visual cue to determine when boat noise may be occurring potentially misses some low-amplitude sources. Thus, the development of a detector based on the distribution of energy across frequencies or on the temporal pattern of boat acoustic energy could increase the variety of sources that can be identified on acoustic recordings.

These are perhaps the first data describing the temporal, spectral, and sound level patterns of small boat noise on coral reefs. The data also provide a novel means of quantifying human usage. While the changes to coral reef soundscapes in the presence of vessel noise may 

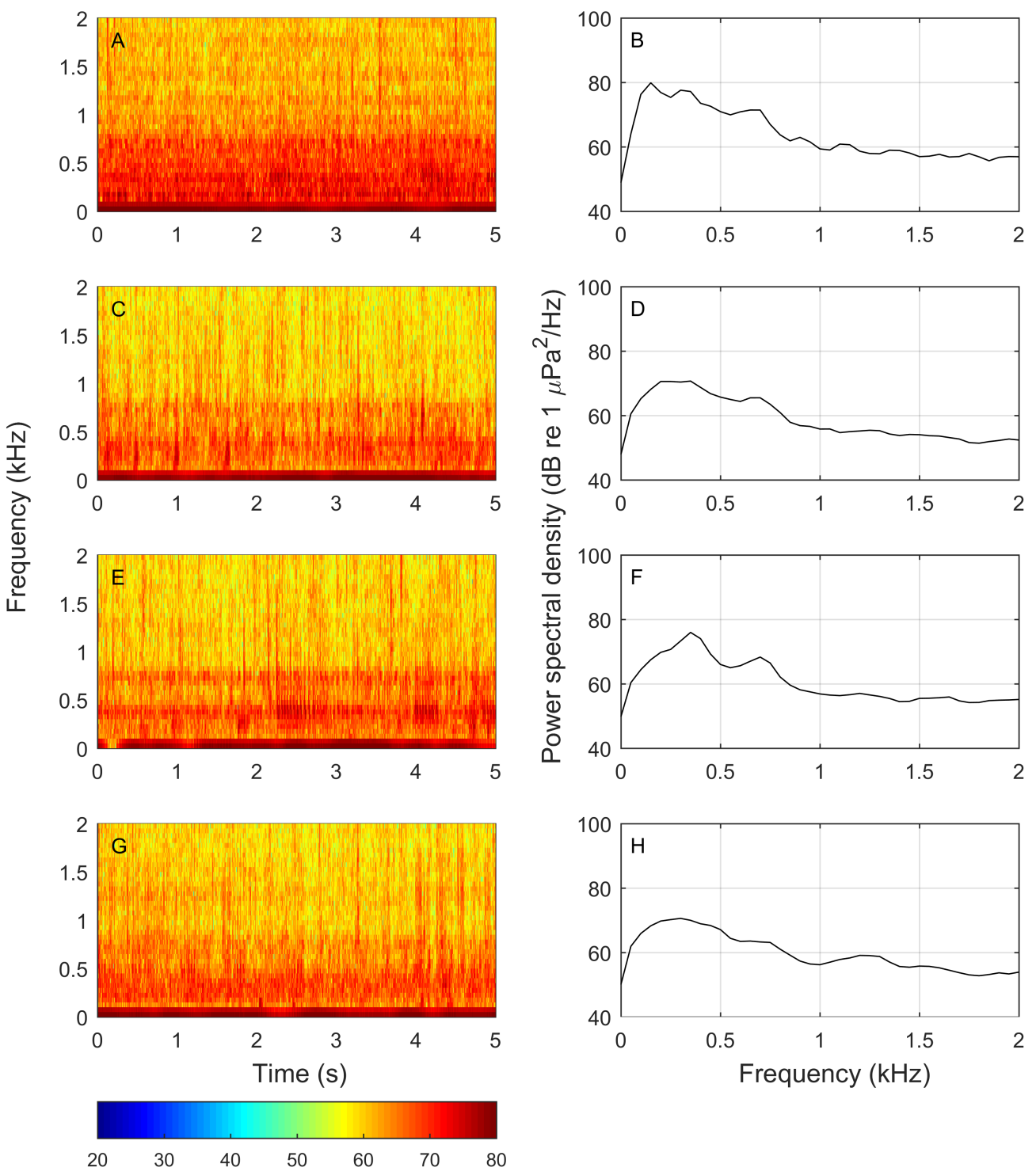

Figure 5.7: Four short clips of boat noise from randomly selected recordings from Tektite at 20:00, the time with most boat activity at that site. While there are differences among these recordings, the spectra follow a similar pattern, with elevated energy below $1 \mathrm{kHz}$. Color bar units are $\mathrm{dB}$ re $1 \mu \mathrm{Pa}$.

be concerning, boat noise may also be used by managers interested in evaluating patterns of area use. Human activity in the marine environment is often challenging to quantify, and these passive acoustic measures could aid in evaluating the ecosystem services that these reefs provide. 


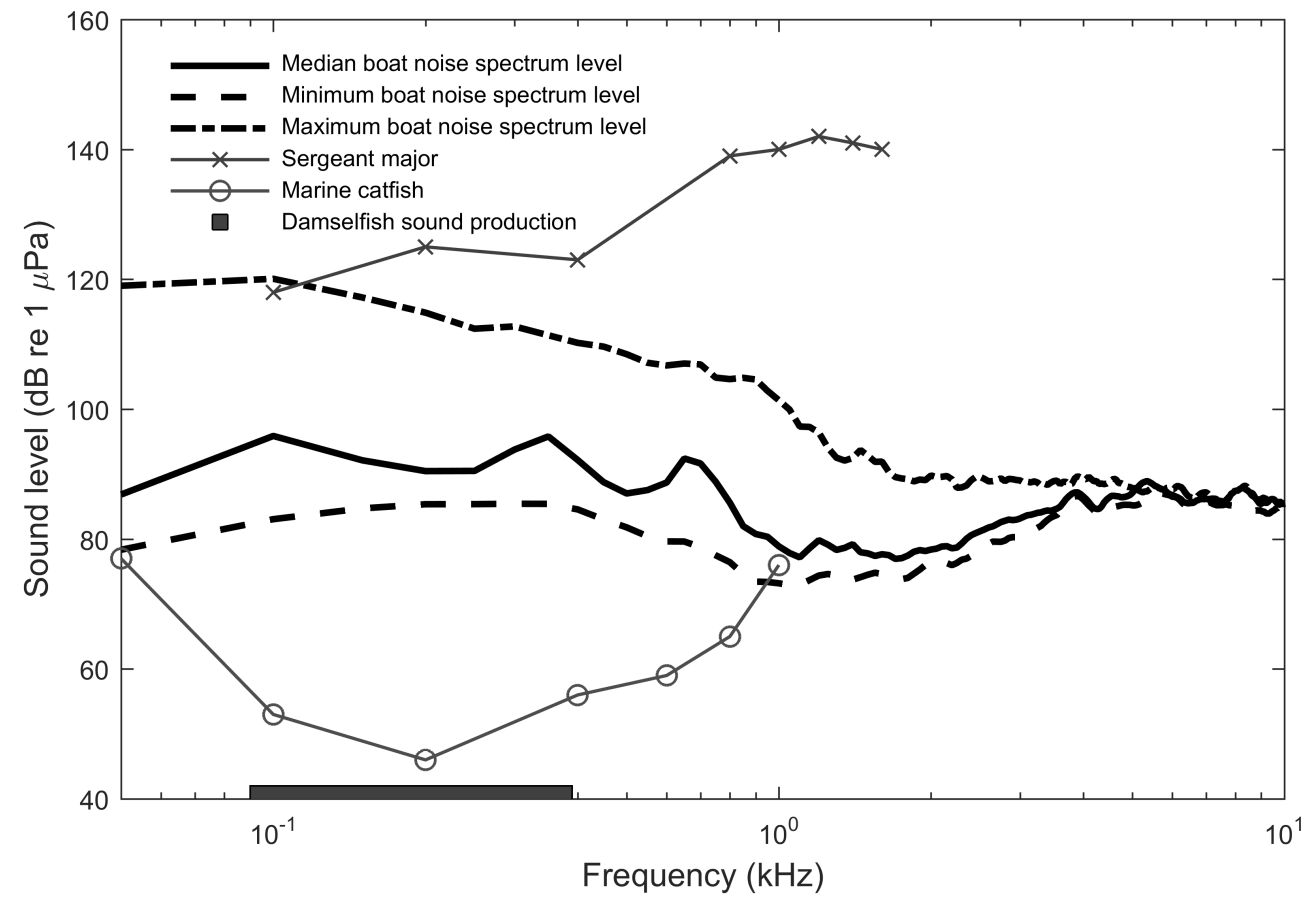

Figure 5.8: Power spectra of the minimum, median, and maximum received levels of boat noise (thick lines), the hearing thresholds for a generalist, the sergeant major (Egner and Mann, 2005), a specialist, the marine catfish (Popper and Tavolga, 1981), and the frequency ranges of damselfish sound production (Maruska et al., 2007). 


\section{Chapter 6}

A coming boom in commercial

shipping? The potential for rapid

growth of noise from commercial

ships by 2030

This chapter was originally published as: Kaplan, M.B. \& Solomon, S. 2016. A coming boom in commercial shipping? The potential for rapid growth of noise from commercial ships by 2030. Marine Policy 73(2016):119-121. DOI: 10.1016/j.marpol.2016.07.024. Used with permission as granted in the original copyright agreement. 


\subsection{Abstract}

Ocean noise levels are thought to be increasing as a result of major growth in global shipping activity, but data quantifying those changes are limited in space and time. As an alternative approach, this study examines the current and future maximum noise capacity of 3 segments of the global commercial shipping fleet: container ships, oil tankers, and bulk carriers. It shows that continued growth in the number of ships, the quantity of goods carried and the distances traveled could increase the maximum noise capacity of the global shipping fleet by up to a factor of 1.9 by 2030, with major growth in particular in the container and bulk carrier segments. Thus, in the absence of operational or manufacturing changes to such ships, the contribution of commercial shipping to ambient ocean noise levels can be expected to dramatically increase.

\subsection{Introduction}

Growth of the world economy can be expected to cause an increase in commercial shipping activity and the attendant potential for ocean noise. There is a great deal of interest in understanding and mitigating ocean noise and its impact on marine life, but quantitative estimates of how ocean noise levels are changing are limited. One study, carried out in the Pacific Ocean off San Nicolas Island, California, found that low-frequency noise levels increased from the mid-1960s to 2000 by up to 3 decibels (dB) per decade, which the authors attributed to commercial shipping activity (McDonald et al., 2006), although more recent estimates suggest that the rate of increase at that location has diminished (Andrew et al., 2011). Other work, based on comparing contemporary recordings made south of Bermuda to historical recordings from the 1960s, has indicated lower but positive rates of increase in low-frequency ambient noise in that part of the North Atlantic (Sirović et al., 2016). Changes to ambient soundscapes elsewhere in the ocean have yet to be fully documented, in large part due to a lack of long-term acoustic monitoring studies. Accordingly, available direct measurements are not sufficient to determine exactly how ocean noise resulting from commercial shipping has changed, and it is clear that many uncertain factors, including operational practices (e.g., slowing down to reduce noise levels), economic growth (e.g., greater or lesser demand for shipping), and recording location can affect actual noise levels (e.g., Frisk, 2012). 
This study takes a different approach by using available information to estimate what will be referred to as the "maximum noise capacity" of the commercial shipping fleet, i.e., an upper limit of the noise level that should be expected in the absence of mitigation measures, with specific focus on oil tankers, bulk carriers, and container ships. It will show that the size of the global fleet has increased rapidly since about 2000 and is projected to continue to grow at a rapid rate, because of the following factors: (i) a higher volume of material is being shipped, particularly as globalization has led to the growth of export industries in Asia; (ii) larger and generally noisier ships are now becoming standard, and (iii) the distance over which material is being transported has grown and is projected to increase further (in particular, as a result of the opening of the Panama Canal Expansion, which will allow larger vessels to pass through). The combination of these factors acting jointly may lead to very rapid rates of increase in the maximum noise capacity of these segments of the commercial fleet.

\subsection{Methods}

Fleet-wide source levels (dB re $1 \mu \mathrm{Pa} @ 1 \mathrm{~m}, 50-1000 \mathrm{~Hz}$ ) for the container, bulk, and oil tanker segments of the global fleet in 2015 and projected forward to the year 2030 were computed as follows. The number of ships in each of these segments was identified from Equasis Statistics reports for the years 2005-2010) (Equasis Statistics) and from UNCTAD Statistics for the years 2011-2015 (UNCTAD Statistics, 2016). The average annual growth rate between 2005 and 2015 was then computed for each segment to make a forward projection of the number of ships per segment in 2030. The average source level for each of these ship types was computed (on the linear scale) based on data contained in McKenna et al. (2012) and was found to be 185.6, 181.0 and $185.7 \mathrm{~dB}$ re $1 \mu \mathrm{Pa} @ 1$ $\mathrm{m}$ for container ships, oil tankers, and bulk carriers respectively. These data were selected because they are based on relatively new ships and represent values that are lower than those presented in earlier estimates (e.g., Arveson and Vendittis, 2000), thus adding a degree of conservatism to these projections. Finally, the projected fleet-wide source levels were computed by multiplying this average source level on the linear scale by the projected number of ships in each segment of the fleet and then returning the source levels to the decibel scale. 
Projections for the quantity of goods carried by distance (measured in ton-miles) were carried out in a similar way and were used to identify the distance traveled by each fleet segment in a given year, assuming that each segment operated at full capacity and keeping capacity fixed at 2015 levels. Using that distance, the segment-wide sound exposure level ( $\mathrm{dB}$ re $1 \mu \mathrm{Pa}^{2} \cdot \mathrm{h}$ ) was calculated using average speeds of $21.3,13.3$, and 14.1 knots for container ships, tankers, and bulk carriers respectively (McKenna et al., 2012) with the average source levels noted above and using the equation $S E L=S L+10 \log _{10}(D / s)$, where $\mathrm{SL}$ is source level, $\mathrm{D}$ is distance in nautical miles, and $\mathrm{s}$ is speed in knots.

\subsection{Results \& Discussion}

Between 1955 and the early 2000s, the global merchant ship fleet (ships over 100 gross tonnage) increased in both the number of ships and in shipping capacity (Figure 6.1) at roughly similar rates. Since then, capacity growth has outpaced fleet growth with the construction of very large tankers and container ships (Figure 6.1).

Projections for the number of ships in the global container, tanker and bulk carrier fleets in 2030, based on the growth rates for each segment over the last decade, suggest that the container and bulk carrier fleets will double over about the next 15 years, while the tanker fleet will undergo a more limited increase (Table 6.1); on average, these 3 segments will contribute to an $87 \%$ increase in ocean noise at the source. Based on these calculations, and assuming no change to the size of ships or their noise characteristics, the growth in fleet size in container ships and bulk carriers would result in more than a doubling in the source levels of these segments.

Further, the projected fleet growth is expected to be dominated by larger vessels (Lloyds Register Group, 2013), which are generally noisier (Arveson and Vendittis, 2000). For example, container ships with capacities greater than 7600 twenty-foot equivalent units (TEU) are projected to increase from $20 \%$ of the container fleet to approximately $48 \%$ by 2030 (Lloyds Register Group, 2013). This increase in large vessels with expected higher source levels (McKenna et al., 2013) represents an amplifier on maximum noise capacity, and suggests that the magnitude of additional noise emissions presented here, which is based on an average source level for each segment of the fleet today, could be an underestimate.

In addition to fleet growth there has been a steady increase in both the quantity of goods 


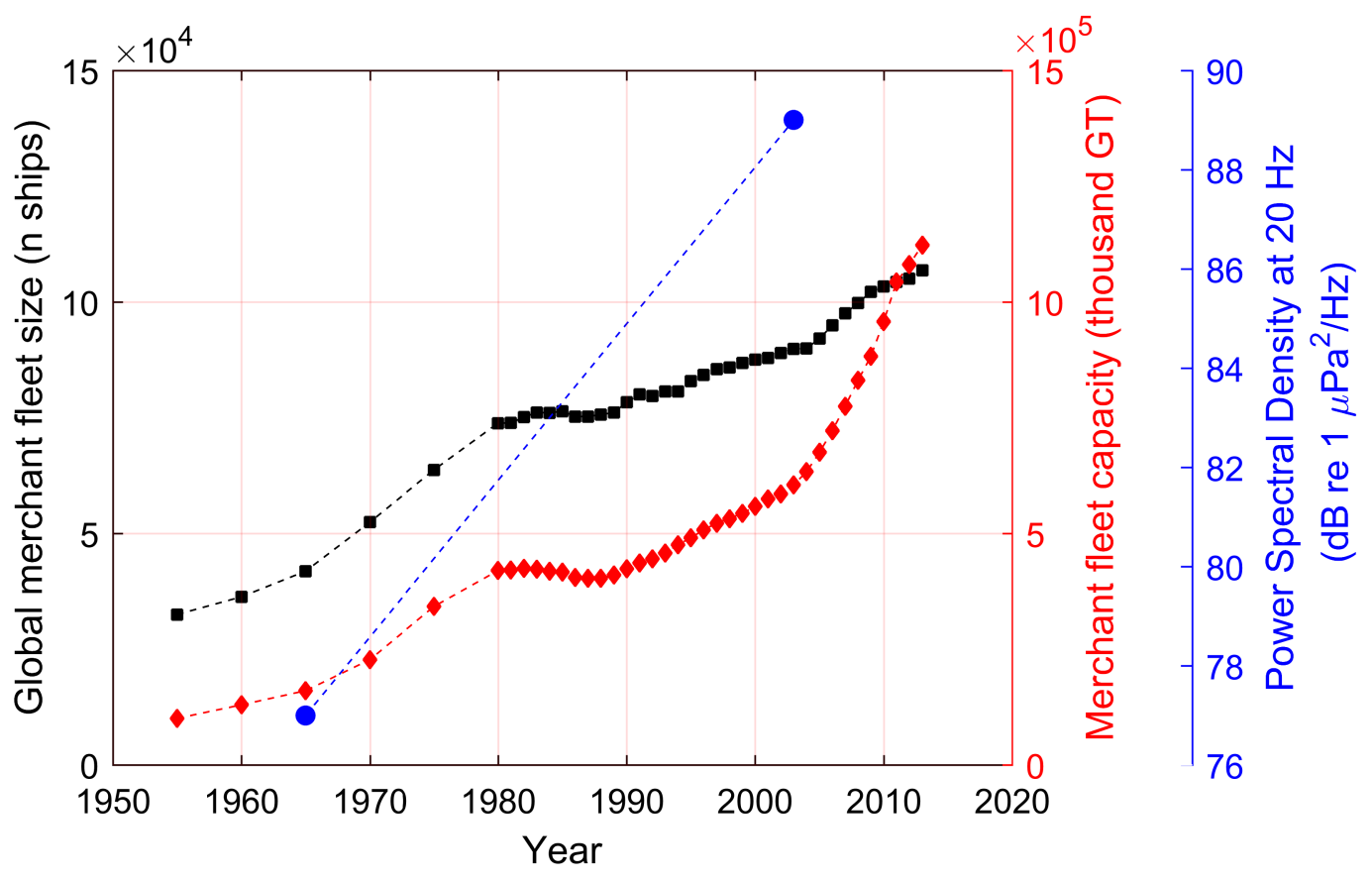

Figure 6.1: Historical size of the global merchant shipping fleet in number of ships (black squares) and fleet capacity in thousand gross tonnage (red diamonds) and 2 measurements of ambient noise levels at $20 \mathrm{~Hz}$ off San Nicolas Island, California (blue circles). In recent years, capacity growth has outpaced growth in vessel numbers as increasingly large ships are built and brought into service. Data from McDonald et al. (2006); Andrew et al. (2011).

shipped and the distances over which they travel. Projections into the future indicate that ton-miles, the distance over which 1 ton of material is carried, will increase substantially. Even if capacity growth were to cease, a major increase in the distance that the existing fleet would travel would be expected, substantially increasing total noise emissions by an average of $102 \%$ (Table 6.2). Conversely, if capacity continues to grow, noise emission levels could stay flat or be reduced if the capacity growth comes from larger ships that are no louder than existing ships. Furthermore, as a result of the completion of the Panama Canal Expansion project, larger (post-Panamax) ships will be traveling further from the Pacific and up the east coast of North America, which will further increase ensonification of the marine environment (Bratton et al., 2015). 
Table 6.1: Data and projections for the number of ships in the container, oil tanker, and bulk carrier fleets and the current estimated and future projected fleet-wide source levels $(50-1000 \mathrm{~Hz}$; dB re $1 \mu \mathrm{Pa} @ 1 \mathrm{~m})$. Data obtained from Equasis Statistics; UNCTAD Statistics (2016); McKenna et al. (2012).

\begin{tabular}{l|l|l|l|l|l|l} 
& $\begin{array}{l}\text { Number of } \\
\text { ships (2015) }\end{array}$ & $\begin{array}{l}\text { Average annual } \\
\text { growth rate } \\
(2005-2015)\end{array}$ & $\begin{array}{l}\text { Projected number } \\
\text { of ships (2030) }\end{array}$ & $\begin{array}{l}\text { Fleet-wide SL } \\
(2015)\end{array}$ & $\begin{array}{l}\text { Projected fleet-wide } \\
\text { SL (2030) }\end{array}$ & $\begin{array}{l}\text { Projected percentage increase } \\
\text { of maximum noise capacity in } \\
2030 \text { relative to 2015 }\end{array}$ \\
\hline Container ships & 5106 & 0.058 & 11971 & 259.8 & 267.2 & 134 \\
Oil tankers & 9435 & 0.007 & 10497 & 260.5 & 261.4 & 11 \\
Bulk carriers & 10340 & 0.053 & 22296 & 266 & 272.7 & 116
\end{tabular}

Table 6.2: Data and projections for the quantity of goods and distance traveled by segment of the fleet in 2015 and projected for 2030 while keeping capacity fixed with the resultant sound exposure levels (SEL 50-1000 Hz; dB re $1 \mu \mathrm{Pa}^{2}$. h). Data obtained from UNCTAD Statistics (2016); McKenna et al. (2012); UNCTAD (2015).

\begin{tabular}{l|l|l|l|l|l|l|l} 
& $\begin{array}{l}\text { Capacity } \\
(2015 ;\end{array}$ & $\begin{array}{l}\text { Ton-miles } \\
\text { thousand dwt) }\end{array}$ & $\begin{array}{l}\text { Average annual } \\
\text { ton-miles growth } \\
\text { rate (2005-2015) }\end{array}$ & $\begin{array}{l}\text { Projected } \\
\text { ton-miles } \\
(2030 ; \text { billions })\end{array}$ & SEL (2015) & $\begin{array}{l}\text { Projected } \\
\text { SEL (2030) }\end{array}$ & $\begin{array}{l}\text { Projected percentage } \\
\text { increase in SEL } \\
\text { in 2030 relative to 2015 }\end{array}$ \\
\hline Container ships & 227740 & 9024 & 0.057 & 20797 & 218.3 & 222 & 53 \\
Oil tankers & 489390 & 12339 & 0.015 & 15312 & 213.8 & 218 & 62 \\
Bulk carriers & 760470 & 16885 & 0.064 & 42811 & 217.7 & 227 & 192
\end{tabular}


Whereas commercial ships primarily contribute to low-frequency ambient sound, small vessel engine amplitudes are highest between $1-5 \mathrm{kHz}$, where sound levels can be between 150 and $180 \mathrm{~dB}$ re $1 \mu \mathrm{Pa} @ 1 \mathrm{~m}$ (Kipple and Gabriele, 2003; Erbe, 2002). Given that sounds at higher frequencies attenuate more rapidly than those at lower frequencies and that small, typically recreational vessels are largely restricted to nearshore coastal environments, noise from small vessels is less likely to contribute to overall ambient noise levels in the oceans and is more likely to be transient. Nevertheless, small vessels can produce nearly continuous noise when they occur at high density in coastal waterways (Haviland-Howell et al., 2007) and have been shown to affect shallow-water soundscapes in significant ways (Kaplan and Mooney, 2015). While this paper focuses primarily on commercial shipping, efforts to study and, if necessary, mitigate the effects of recreational vessel noise are also needed.

\subsection{Conclusions}

This study has shown that the global ocean's maximum noise capacity from 3 major segments of commercial shipping can be expected to increase by $87-102 \%$ on average by 2030 , due to the combined effects of increased shipping, larger and noisier ships, and increased shipping distances - a 'perfect storm' of amplifying factors. Changes to operational practices, such as speed reductions, might help reduce ocean noise, at least in the most vulnerable areas and as

long as capacity is not simultaneously increased. Noise levels could be curbed if quieter ships were brought into the fleet. Voluntary guidelines on ship noise ratified by the International Maritime Organization in 2014 (IMO Marine Environment Protection Committee, 2013) could be made regulatory, which would push shipping companies to only purchase quieter ships. This could be a phased requirement for new construction and/or retrofits. Such a regulatory shift would also require the introduction of standardized ship noise measurement procedures for ports, similar to how airports currently monitor commercial aircraft noise (Falzone, 1999). Such port measurement schemes are already being introduced on an ad hoc basis (Port of Vancouver, 2015). Because ships and canals will be operated for many years, choices being made now will affect the ocean for decades to come. The evaluation of the maximum noise capacity as presented here highlights the acute challenge facing the world's oceans and the value of prompt mitigation. 
THIS PAGE INTENTIONALLY LEFT BLANK 


\section{Chapter 7}

\section{Conclusion}




\subsection{Synthesis}

The ubiquity of sound-producing organisms in the marine environment has long been known, but only recently have efforts to quantify the rich diversity of soniferous species become common. In no habitat has this been more important than coral reefs, the acoustic environments of which contain sounds produced by a broad range of invertebrate, fish and occasionally marine mammal species (Au et al., 2012). Because sounds vary in their spectral and temporal attributes they can often be attributed to taxa and sometimes species (e.g., Tricas and Boyle, 2014). As a result, broad patterns of sound production are likely indicative of the species assemblages present. On a more granular level, because sound production is often paired with behaviors in a predictable way (e.g., Mann and Lobel, 1997), understanding the diversity and patterns of occurrence of sounds might provide a high-resolution window into reef ecology. Such possibilities are promising; however, there appears to be considerable variability in space and time in the sounds produced in a given habitat (Radford et al., 2008, 2014; Staaterman et al., 2014, 2013; Nedelec et al., 2015). Accordingly, investigations distilling that variability into representative summary values and relating those values to the species assemblages present on reefs are necessary precursors to using passive acoustic monitoring of reef sound as a method to eavesdrop on reefs.

Reefs are dynamic not only acoustically but also in the composition of their species assemblages (Graham et al., 2006). Many factors play a role, including natural seasonal variability, rarer cyclical events such as El Ninos, and changing environmental conditions over longer periods of time. For reefs to survive, larval recruitment is critical, as juvenile animals are needed to replenish aging populations. Many reef animals have a pelagic larval phase; however, the methods by which larval animals identify suitable adult habitat have yet to be comprehensively elucidated (Kingsford et al., 2002). One of the potential sensory modalities by which larval animals might mediate adult habitat selection is hearing (Kingsford et al., 2002). Indeed, a number of studies have provided evidence for orientation to reef sound in larval fishes (e.g., Simpson et al., 2008) but the spatial range over which this cue might operate has yet to be satisfactorily assessed because the distance over which a reef soundscape might propagate will likely depend on a number of different factors (Mann et al., 2007; Radford et al., 2011b). As a result of the complex bathymetry and non-point-source nature of reefs as sound sources, empirical measurements of acoustic propagation at a range of 
distances are needed to estimate the range over which sound might be a useful navigation cue for marine larvae (Nedelec et al., 2016).

Sounds produced by marine fauna are not the only acoustic sources in the oceans; indeed, anthropogenic noise is increasingly prevalent in both the open ocean and in coastal reef environments (S̊irović et al., 2016; Haviland-Howell et al., 2007). While there have been some assessments of the changes to ambient low-frequency sound levels in the deep ocean over time as a result of commercial shipping activity (e.g., McDonald et al., 2006), these efforts have been relatively sparse and similar assessments for shallow-water habitats have been even more limited. Acoustic recordings of coral reef environments inevitably contain anthropogenic noise; accordingly, assessing the extent to which there is spectral and temporal overlap between biological signals of interest and noise is a necessary first step to understanding how this noise might affect underwater acoustic communication.

To provide clarity on these issues, this thesis sought to unearth the drivers of spatiotemporal variability in sound production, link that variability to species assemblages present on reefs, understand how far reef sounds might travel, assess the overlap between small boat noise and reef sound, and produce projections of how ocean noise levels resulting from commercial shipping activity might change in the future.

In chapter 2, I studied the soundscapes of 3 reefs located in the U.S. Virgin Islands National Park. I identified a positive correlation between the magnitude of the diel increase in low-frequency sound production and biological attributes such as fish density and coral cover on those reefs. In contrast, there was no similar correlation between high-frequency sound levels and species assemblage characteristics. This divergent result made sense intuitively - reefs with larger soniferous fish assemblages and/or more available habitat would be expected to exhibit greater increases in low-frequency sound level at the crepuscular time, whereas because snapping shrimp can likely live in a broader range of habitats (i.e., they are not dependent on live coral cover as some fish species are), it is perhaps expected that those high-frequency levels do not correlate with the observed reef fauna. However, these results were based on a relatively short recording period (ca. 4 mo) and sample size (3 reefs); accordingly, a more comprehensive assessment was desirable.

In chapter 3, I executed a broader investigation. I recorded 7 reefs for a ca. 16 month period in Maui, Hawaii, to better parameterize the biological drivers of sound production on reefs. Results from this study confirmed that the metric developed in chapter 2 to quantify 
the extent of sound production at the community level - the strength of the diel trend was suitable to characterize these Hawaiian reefs; however, the relationship between fish assemblages, benthic cover and this acoustic parameter was non-linear, underscoring the fact that a multiplicity of drivers may be implicated in influencing coral reef soundscapes. Chapters 2 and 3 identified broad relationships between reef biota and the local biological soundscape. However, soundscapes were also influenced by the local environment of the reef, and the correlation identified in chapter 2 did not hold in chapter 3 . This suggests that the ultimate goal of inverse prediction of reef species assemblages based on acoustics alone is not yet fully possible, at least not using the metrics presented here.

Given the variability within and among reefs in sound level, it is likely that the distances over which those sounds can be detected by marine fauna will also vary. Thus, in chapter 4 I quantified the ranges over which sound emanating from a Hawaiian reef might travel and compared that distance and associated sound level to published fish hearing thresholds. Crucially, this study measured both sound pressure and particle motion at a range of distances, times of day, and on 3 separate days. Because reefs are not point-sources and are bathymetrically complex and because there is uncertainty around the near-field/far-field barrier, direct measurement of both acoustic quantities, and particularly particle motion, the relevant acoustic stimulus for most sound-sensitive marine invertebrates and fishes, was needed. This chapter indicated that the study reef's average soundscape would likely not be detectable at any distance from the reef, and in general was too faint to be heard, even at the source, by most organisms for whom hearing data are available. These results may call into question the widely held notion that sound could play a role in orientation behavior for reef larvae; conversely, the presence of high-amplitude transient sounds suggests that detection might be possible even if the average levels are too low to be heard.

Increasingly, biological sounds are not the dominant acoustic sources on reefs. Indeed, as chapter 5 demonstrates, small boat noise can be highly prevalent and can cause significant spectral and amplitude changes to ambient soundscapes. Patterns of boat noise occurrence mirrored patterns of human activity (i.e., elevated levels during the day compared to at night) and the presence of vessel noise was not uniformly distributed among study reefs. Such assessments are disappointingly rare in the literature but provide critical data for identifying the overlap between vessels and marine animal hearing and sound production.

While small-boat noise is largely confined to coastal areas, open ocean noise levels at 
low frequencies may have increased substantially over the last several decades as a result of commercial shipping and other anthropogenic activity. In chapter 6 , I conducted a modeling exercise which demonstrated that if current trends in commercial shipping capacity and ship size continue, the source level of the commercial fleet could double by 2030 . These results suggest that mitigation in the form of technological advances (i.e., quieter ships) combined with regulatory action might be needed.

\subsection{Next steps}

There are several engaging opportunities to build on the research presented in this thesis. The apparent inability at present to use acoustic data to generate inverse predictions of reef species assemblages is disappointing and curious. One reason for this might be that a broader range of reefs that are highly similar in bathymetry and propagation conditions but with more diverse species assemblages are needed for more meaningful acoustic differences to emerge among reefs. Follow-up studies should endeavor to record reefs that cover a wide range of species assemblages including diversity in size classes of soniferous fishes. It is also possible that there are summary parameters other than the ones developed and employed here that could provide additional or complementary information about community-level patterns of sound production. Further examination of a broad range of potential parameters is thus warranted. Alternatively, it is possible that broad-scale community-level summaries, such as the ones presented here, mask nuanced differences among reefs that might emerge from more fine-scale assessments that examine and quantify the sounds being produced, and not just the overall trend.

Indeed, the broad trends in reef soundscapes have now been described for several reef systems; however, more granular investigations looking at the occurrence patterns of individual sound types are far rarer (but see Ruppe et al., 2015). Such studies are labor intensive when manual identification of sounds is required. Nevertheless, it is possible that the use of automatic detection and classification algorithms might assist in identifying the temporal occurrence and abundance of calls by type. Such data, when paired with quantitative visual surveys, might allow researchers to characterize call rates and, ultimately, make density estimates based on call occurrence patterns, if the identity of the sound-producing organism is known. Considerable effort has gone into attempts to produce density estimates of 
cetaceans using acoustic data; those efforts could be harnessed to make similar estimates for fish populations, perhaps in real time, which would benefit resource managers. A few caveats are, however, in order. Acoustic density estimation can be done only for species that have highly stereotyped sounds that are discriminable from the background noise. In addition, the context in which sounds are produced is important - if, for example, a given sound is produced only during spawning, detection of that sound would be a useful indicator of spawning behavior in that species but would not necessarily indicate presence other than at that time. Identifying the species that produced a specific sound is highly labor intensive. Catalogues that characterize sound production behavior ought to be made publicly available such that any interested researcher can use them for density estimation or other purposes.

The results from chapter 4 suggest that the overall soundscape may not be detectable far from the reef. Additional studies should collect similar particle motion propagation data from a wider range of reefs and during multiple seasons, as results may vary. Furthermore, there is a striking dearth of hearing data available for the majority of fish species and almost none for larval or juvenile fishes; such data are needed to make inferences about the ranges over which reef sounds might be detectable by those species. This is a ripe area of research, as knowledge of fish hearing thresholds will also be critical to assessing the extent to which anthropogenic noise might affect reef fish physiology and behavior.

Indeed, the effects of anthropogenic noise on aquatic animals are largely unknown, although recent work has attempted to uncover some potential implications such as elevated mortality rates in the presence of small boat noise (Simpson et al., 2016). Further studies should examine whether similar effects manifest in other taxa. Studying the effects of acute stressors is relatively straightforward; conversely, uncovering the impacts of chronic stressors such as elevated ambient noise levels is much more difficult. Experimental paradigms to better quantify chronic effects, especially on vital rates, are as urgently needed as they are difficult to design and execute.

One of the major contributors to ambient ocean noise levels is commercial shipping, which has increased substantially since the mid-1950s. There are very few systems in place at present to assess the source levels of commercial ships, which makes quantifying the overall contribution of this industry to ocean noise levels challenging. More comprehensive assessments of ship source level characteristics are needed to better parameterize noise levels at the source and to identify which ships are making outsize noise contributions. Such data 
could be used in regulatory frameworks to incentivize the use of quieter ships.

In conclusion, this thesis has expanded what is currently known about marine soundscapes and the contributions of both biological and anthropogenic sources. It has left open several avenues for further bioacoustic research. With the proliferation and decreasing cost of acoustic recorders and advances in remote sensing technologies, this field is likely to continue to grow and provide increasingly granular and valuable insight into reef ecology, anthropogenic activity, and the effects of a changing environment. 
THIS PAGE INTENTIONALLY LEFT BLANK 


\section{Bibliography}

A. S. Ackleh, G. E. Ioup, J. W. Ioup, B. Ma, J. J. Newcomb, N. Pal, N. A. Sidorovskaia, and C. Tiemann. Assessing the Deepwater Horizon oil spill impact on marine mammal population through acoustics: endangered sperm whales. J Acoust Soc Am, 131(3):23062314, 2012.

M. C. P. Amorim. Diversity of sound production in fish. In F. Ladich, S. P. Collin, P. Moller, and B. G. Kapoor, editors, Communication in Fishes, chapter 3, pages 71-105. Science Pub Inc, Boca Raton, FL, 2006.

R. K. Andrew, B. M. Howe, and J. A. Mercer. Long-time trends in ship traffic noise for four sites off the North American West Coast. J Acoust Soc Am, 129(2):642-651, 2011.

P. T. Arveson and D. J. Vendittis. Radiated noise characteristics of a modern cargo ship. $J$ Acoust Soc Am, 107(1):118-129, 2000.

J. Atema. Aquatic odor dispersal fields: opportunities and limits of detection, communication and navigation. In C. Brönmark and L. A. Hansson, editors, Chemical Ecology in Aquatic Systems, chapter 1, pages 1-18. Oxford University Press, Oxford, UK, 2012.

J. Atema, M. J. Kingsford, and G. Gerlach. Larval reef fish could use odour for detection, retention and orientation to reefs. Mar Ecol Prog Ser, 241:151-160, 2002.

W. W. Au, M. Richlen, and M. O. Lammers. Soundscape of a nearshore coral reef near an urban center. In A. N. Popper and A. Hawkins, editors, The Effects of Noise on Aquatic Life, volume 730, pages 345-351. Springer Science and Business Media, New York, NY, 2012.

W. W. L. Au and K. Banks. The acoustics of the snapping shrimp Synalpheus parneomeris in Kaneohe Bay. J Acoust Soc Am, 103(1):41-47, 1998.

J. Beets and A. M. Friedlander. Evaluation of a conservation strategy: a spawning aggregation closure for red hind, Epinephelus guttatus, in the U.S. Virgin Islands. Environ Biol Fishes, 55:91-98, 1998.

D. R. Bellwood, T. P. Hughes, C. Folke, and M. Nystrom. Confronting the coral reef crisis. Nature, 429:827-833, 2004.

F. Bertucci, E. Parmentier, G. Lecellier, A. D. Hawkins, and D. Lecchini. Acoustic indices provide information on the status of coral reefs: an example from Moorea Island in the South Pacific. Sci Rep, 6:33326, 2016. 
D. R. Bohnenstiehl, A. Lillis, and D. B. Eggleston. The curious acoustic behavior of estuarine snapping shrimp: Temporal patterns of snapping shrimp sound in sub-tidal oyster reef habitat. PLoS One, 11(1):e0143691, 2016.

J. Bratton, D. Burke, P. Ulrich, S. Raetz, and S. Laxmana. How the panama canal is redrawing the logistics map. Report, BCG Perspectives, June 162015 2015. URL https://www.bcgperspectives.com/content/articles/transportationtravel-tourism-how-panama-canal-expansion-is-redrawing-logistics/.

R. E. Brock. A critique of the visual census method for assessing coral reef fish populations. Bull Mar Sci, 32(1):269-276, 1982.

J. F. Bruno, L. E. Petes, C. D. Harvell, and A. Hettinger. Nutrient enrichment can increase the severity of coral diseases. Ecol Lett, 6:1056-1061, 2003.

J. F. Bruno, E. R. Selig, K. S. Casey, C. A. Page, B. L. Willis, C. D. Harvell, H. Sweatman, and A. M. Melendy. Thermal stress and coral cover as drivers of coral disease outbreaks. PLoS Biology, 5(6):e124, 2007.

B. U. Budelmann. Hearing in non-arthropod invertebrates. In D. B. Webster, R. R. Fay, and A. N. Popper, editors, The Evolutionary Biology of Hearing, chapter 10, pages 141-155. Springer-Verlag, New York, NY, 1992a.

B. U. Budelmann. Hearing in crustacea. In D. B. Webster, R. R. Fay, and A. N. Popper, editors, The Evolutionary Biology of Hearing, chapter 9, pages 131-140. Springer-Verlag, New York, NY, 1992b.

D. H. Cato. Marine biological choruses observed in tropical waters near Australia. J Acoust Soc Am, 64(3):736-743, 1978.

D. H Cato. Some unusual sounds of apparent biological origin responsible for sustained background noise in the Timor Sea. J Acoust Soc Am, 68(4):1056-1060, 1980.

D. H. Cato and M. J. Bell. Ultrasonic ambient noise in australian shallow waters at frequencies up to 200 kHz. Report MRL-TR-91-23, Materials Research Laboratory, DSTO, Australia, 1992.

N. R. Chapman and A. Price. Low frequency deep ocean ambient noise trend in the Northeast Pacific Ocean. J Acoust Soc Am, 129(5):EL161-5, 2011.

K. M. Chong-Seng, T. D. Mannering, M. S. Pratchett, D. R. Bellwood, and N. A. Graham. The influence of coral reef benthic condition on associated fish assemblages. PLoS One, 7 (8):e42167, 2012.

A. Codarin, L. E. Wysocki, F. Ladich, and M. Picciulin. Effects of ambient and boat noise on hearing and communication in three fish species living in a marine protected area (Miramare, Italy). Mar Pollut Bull, 58(12):1880-1887, 2009.

A. B. Coffin, D. G. Zeddies, R. R. Fay, A. D. Brown, P. W. Alderks, A. A. Bhandiwad, R. A. Mohr, M. D. Gray, P. H. Rogers, and J. A. Sisneros. Use of the swim bladder and lateral line in near-field sound source localization by fish. J Exp Biol, 217:2078-2088, 2014. 
T. M. Cox, T. J. Ragen, A. J. Read, E. Vos, R. W. Baird, K. Balcomb, J. Barlow, J. Caldwell, T. Cranford, and K. Crum. Understanding the impacts of anthropogenic sound on beaked whales. J Cetacean Res Manage, 7(3):177-187, 2006.

E. D'Alessandro, Su Sponaugle, and T. Lee. Patterns and processes of larval fish supply to the coral reefs of the upper Florida Keys. Mar Ecol Prog Ser, 331:85-100, 2007.

G. De'ath and K. E. Fabricius. Classification and regression trees: a powerful yet simple technique for ecological data analysis. Ecology, 81(11):3178-3192, 2000.

G. De'ath, K. E. Fabricius, H. Sweatman, and M. Puotinen. The 27-year decline of coral cover on the Great Barrier Reef and its causes. Proc Natl Acad Sci U S A, 109(44): 17995-17999, 2012.

M. Depraetere, S. Pavoine, F. Jiguet, A. Gasc, S. Duvail, and J. Sueur. Monitoring animal diversity using acoustic indices: Implementation in a temperate woodland. Ecol Indic, 13 (1):46-54, 2012 .

L. Di Iorio and C. W. Clark. Exposure to seismic survey alters blue whale acoustic communication. Biol Lett, 6(1):51-54, 2010.

P. Doherty and T. Fowler. An empirical test of recruitment limitation in a coral reef fish. Science, 263:935-939, 1994.

K.O. Dzhaparidze. Parameter estimation and hypothesis testing in spectral analysis of stationary time series. Springer-Verlag, New York, 1986.

P. J. Edmunds. Decadal-scale changes in the community structure of coral reefs of St. John, US Virgin Islands. Mar Ecol Prog Ser, 489:107-123, 2013.

S. A. Egner and D. A. Mann. Auditory sensitivity of sergeant major damselfish Abudefduf saxatilis from post-settlement juvenile to adult. Mar Ecol Prog Ser, 285:213-222, 2005.

Equasis Statistics. The world merchant fleet - statistics from equasis. Report. URL http://www. emsa. europa.eu/implementation-tasks/equasis-a-statistics/items . html? cid=95\&id=472\.

C. Erbe. Underwater noise of whale-watching boats and potential effects on killer whales (Orcinus orca), based on an acoustic impact model. Mar Mam Sci, 18(2):394-418, 2002.

K. L. Falzone. Airport noise pollution: Is there a solution in sight? Boston College Environmental Affairs Law Review, 26(4):769-807, 1999.

J. L. Fewtrell and R. D. McCauley. Impact of air gun noise on the behaviour of marine fish and squid. Mar Pollut Bull, 64(5):984-993, 2012.

A. M. Friedlander, E. K. Brown, P. L. Jokiel, W. R. Smith, and K. S. Rodgers. Effects of habitat, wave exposure, and marine protected area status on coral reef fish assemblages in the Hawaiian archipelago. Coral Reefs, 22(3):291-305, 2003.

G. V. Frisk. Noiseonomics: the relationship between ambient noise levels in the sea and global economic trends. Sci Rep, 2:437, 2012. 
A. Gasc, J. Sueur, F. Jiguet, V. Devictor, P. Grandcolas, C. Burrow, M. Depraetere, and S. Pavoine. Assessing biodiversity with sound: Do acoustic diversity indices reflect phylogenetic and functional diversities of bird communities? Ecol Indic, 25:279-287, 2013.

G. Gerlach, J. Atema, M. J. Kingsford, K. P. Black, and V. Miller-Sims. Smelling home can prevent dispersal of reef fish larvae. Proc Natl Acad Sci U S A, 104(3):858-863, 2007.

C. Goodall, C. Chapman, and D. Neil. The acoustic response threshold of the Norway lobster, Nephrops norvegicus (1.) in a free sound field. In K. Weise, editor, Frontiers in Crustacean Neurobiology, pages 106-113. Springer, Basel, Switzerland, 1990.

N. A. Graham, D. R. Bellwood, J. E. Cinner, T. P. Hughes, A. V. Norstrom, and M. Nystrom. Managing resilience to reverse phase shifts in coral reefs. Front Ecol Environ, 11(10):541$548,2013$.

N. A. J. Graham and K. L. Nash. The importance of structural complexity in coral reef ecosystems. Coral Reefs, 32:315-326, 2013.

N. A. J. Graham, S. K. Wilson, S. Jennings, N. V. Polunin, J. P. Bijoux, and J. Robinson. Dynamic fragility of oceanic coral reef ecosystems. Proc Natl Acad Sci U S A, 103(22): 8425-8429, 2006.

D. H. Green and P. J. Edmunds. Spatio-temporal variability of coral recruitment on shallow reefs in St. John, US Virgin Islands. J Exp Mar Biol Ecol, 397(2):220-229, 2011.

C. D. Harvell, K. Kim, J. M. Burkholder, R. R. Colwell, P. R. Epstein, D. J. Grimes, E. E. Hofmann, E. K. Lipp, A. D. M. E. Osterhaus, R. M. Overstreet, J. W. Porter, G. W. Smith, and G. R. Vasta. Emerging marine diseases - climate link and anthropogenic factors. Science, 285:1505-1510, 1999.

L. Hatch, C. Clark, R. Merrick, S. Van Parijs, D. Ponirakis, K. Schwehr, M. Thompson, and D. Wiley. Characterizing the relative contributions of large vessels to total ocean noise fields: a case study using the Gerry E. Studds Stellwagen Bank National Marine Sanctuary. Environ Manage, 42(5):735-752, 2008.

G. Haviland-Howell, A. S. Frankel, C. M. Powell, A. Bocconcelli, R. L. Herman, and L. S. Sayigh. Recreational boating traffic: a chronic source of anthropogenic noise in the Wilmington, North Carolina Intracoastal Waterway. J Acoust Soc Am, 122(1):151-160, 2007.

J. Hedley, C. Roelfsema, I. Chollett, A. Harborne, S. Heron, S. Weeks, W. Skirving, A. Strong, C. Eakin, T. Christensen, V. Ticzon, S. Bejarano, and P. Mumby. Remote sensing of coral reefs for monitoring and management: A review. Remote Sens, 8(2):118, 2016 .

G. S. Helfman, B. B. Collette, D.E. Facey, and B. W. Bowen. The diversity of fishes. Wiley-Blackwell, Chichester, UK, 2nd edition, 2009.

J. Herberholz and B. Schmitz. Flow visualisation and high speed video analysis of water jets in the snapping shrimp (Alpheus heterochaelis). J Comp Physiol A, 185:41-49, 1999.

J. A. Hildebrand. Impacts of anthropogenic sound on cetaceans. Report IWC/SC/56/E13, 2004. 
J. A. Hildebrand. Anthropogenic and natural sources of ambient noise in the ocean. Mar Ecol Prog Ser, 395:5-20, 2009.

O. Hoegh-Guldberg, P. J. Mumby, A. J. Hooten, Robert S. Steneck, P. Greenfield, E. Gomez, C. D. Harvell, P. F. Sale, A. J. Edwards, K. Caldiera, N Knowlton, M. Eakin, R. InglesiasPrieto, N. Muthiga, Roger H. Bradbury, A. Dubi, and M. E. Hatziolos. Coral reefs under rapid climate change and ocean acidification. Science, 318:1737-1742, 2007.

G. Hofmann, C. Blanchette, E. Rivest, and L. Kapsenberg. Taking the pulse of marine ecosystems: The importance of coupling long-term physical and biological observations in the context of global change biology. Oceanography, 26(3):140-148, 2013.

S. Holles, S. D. Simpson, A. N. Radford, L. Berten, and D. Lecchini. Boat noise disrupts orientation behaviour in a coral reef fish. Mar Ecol Prog Ser, 485:295-300, 2013.

A. Z. Horodysky, R. W. Brill, M. L. Fine, J. A. Musick, and R. J. Latour. Acoustic pressure and particle motion thresholds in six sciaenid fishes. J Exp Biol, 211:1504-11, 2008.

K. B. Huebert, R. K. Cowen, and S. Sponaugle. Vertical migrations of reef fish larvae in the Straits of Florida and effects on larval transport. Limnol Oceanogr, 56(5):1653-1666, 2011.

T. P. Hughes, A. H. Baird, D. R. Bellwood, M. Card, S. R. Connolly, C. Folke, R. Grosberg, O. Hoegh-Guldberg, J. B. C. Jackson, J. Kleypas, J. M. Lough, P. Marshall, M. Nyström, S. R. Palumbi, J. M. Pandolfi, B. Rosen, and J. Roughgarden. Climate change, human impacts, and the resilience of coral reefs. Science, 301(5635):929-933, 2003.

IMO Marine Environment Protection Committee. Noise from commercial shipping and its adverse impacts on marine life. Report MEPC 66/17, International Maritime Organization, 2013.

J. O. Irisson, C. B. Paris, C. Guigand, and S. Planes. Vertical distribution and ontogenetic "migration" in coral reef fish larvae. Limnol Oceanogr, 55(2):909-919, 2010.

C. E. Johnston and S. P. Vives. Sound production in Codoma ornata (Girard) (Cyprinidae). Environ. Biol. Fishes, 68:81-85, 2003.

P. L. Jokiel and S. L. Coles. Response of Hawaiian and other Indo-Pacific reef corals to elevated temperature. Coral Reefs, 8:155-162, 1990.

G. P. Jones, M. I. McCormick, M. Srinivasan, and J. V. Eagle. Coral decline threatens fish biodiversity in marine reserves. Proc Natl Acad Sci U S A, 101(21):8251-8253, 2004.

M. B. Kaplan and T. A. Mooney. Ambient noise and temporal patterns of boat activity in the US Virgin Islands National Park. Mar Pollut Bull, 98(2015):221-228, 2015.

M. B. Kaplan, T. A. Mooney, J. Partan, and A. R. Solow. Coral reef species assemblages are associated with ambient soundscapes. Mar Ecol Prog Ser, 533:93-107, 2015.

D. Kastak, R. J. Schusterman, B. L. Southall, and C. J. Reichmuth. Underwater temporary threshold shift unduced by octave-band noise in three species of pinniped. J Acoust Soc Am, 106(2):1142-1148, 1999. 
E. V. Kennedy, M. W. Holderied, J. M. Mair, H. M. Guzman, and S. D. Simpson. Spatial patterns in reef-generated noise relate to habitats and communities: Evidence from a Panamanian case study. J Exp Mar Biol Ecol, 395(1-2):85-92, 2010.

M. J. Kingsford, J. M. Leis, A. Shanks, K. C. Lindeman, S. G. Morgan, and J. Pineda. Sensory environments, larval abilities and local self-recruitment. Bull Mar Sci, 70(1): 309-340, 2002.

B. Kipple and C. Gabriele. Glacier bay watercraft noise. Report NSWCCD-71-TR-2003/522, 2003.

N. Knowlton, R. E. Brainard, R. Fisher, M. Moews, L. Plaisance, and M. J. Caley. Coral reef biodiversity. In A. D. McIntyre, editor, Life in the World's Oceans: Diversity, Distribution, and Abundance, number 4, pages 65-78. Wiley-Blackwell, Chichester, UK, 2010.

V. Komyakova, P. L. Munday, and G. P. Jones. Relative importance of coral cover, habitat complexity and diversity in determining the structure of reef fish communities. PLoS One, 8(12):e83178, 2013.

K. J. Kroeker, R. L. Kordas, R. Crim, I. E. Hendriks, L. Ramajo, G. S. Singh, C. M. Duarte, and J. P. Gattuso. Impacts of ocean acidification on marine organisms: quantifying sensitivities and interaction with warming. Glob Chang Biol, 19(6):1884-1896, 2013.

H. P. Kunc, K. E. McLaughlin, and R. Schmidt. Aquatic noise pollution: implications for individuals, populations, and ecosystems. Proc R Soc B, 283(1836):20160839, 2016.

F. Ladich. Effects of noise on sound detection and acoustic communication in fishes. In H. Brumm, editor, Animal Communication and Noise, number 4, pages 65-90. SpringerVerlag, Berlin, Germany, 2013.

F. Ladich and R. R. Fay. Auditory evoked potential audiometry in fish. Rev Fish Biol Fisheries, 23:317-364, 2013.

M. O. Lammers, R. E. Brainard, W. W. Au, T. A. Mooney, and K. B. Wong. An ecological acoustic recorder (EAR) for long-term monitoring of biological and anthropogenic sounds on coral reefs and other marine habitats. J Acoust Soc Am, 123(3):1720-1728, 2008.

J. M. Leis. Are larvae of demersal fishes plankton or nekton? Adv Mar Biol, 51:57-141, 2006.

J. M. Leis and B. M. Carson-Ewart. Orientation of pelagic larvae of coral-reef fishes in the ocean. Mar Ecol Prog Ser, 252:239-253, 2003.

J. M. Leis and M. M. Lockett. Localization of reef sounds by settlement-stage larvae of coral-reef fishes (Pomacentridae). Bull Mar Sci, 76(3):715-724, 2005.

J. M. Leis, H. P. A. Sweatman, and S. E. Reader. What the pelagic stages of coral reef fishes are doing out in blue water: Daytime field observations of larval behavioural capabilities. Mar Freshwater Res, 47:401-411, 1996.

A. Lillis, D. B. Eggleston, and D. R. Bohnenstiehl. Soundscape variation from a larval perspective: the case for habitat-associated sound as a settlement cue for weakly swimming estuarine larvae. Mar Ecol Prog Ser, 509:57-70, 2014. 
Lloyds Register Group. Global marine trends 2030. Report, 2013. URL http://www.lr. org/en/projects/global-marine-trends-2030.aspx/.

P. S. Lobel. Sounds produced by spawning fishes. Environ Biol Fishes, 33:351-358, 1992.

J. V. Locascio and D. A. Mann. Effects of Hurricane Charley on fish chorusing. Biol Lett, $1(3): 362-365,2005$.

J. J. Luczkovich, D. A. Mann, and R. A. Rountree. Passive acoustics as a tool in fisheries science. T Am Fish Soc, 137(2):533-541, 2008.

D. A. Mann and P. S. Lobel. Propagation of damselfish (Pomacentridae) courtship sounds. $J$ Acoust Soc Am, 101(6):3783-3791, 1997.

D. A. Mann, B. M. Casper, K. S. Boyle, and T. C. Tricas. On the attraction of larval fishes to reef sounds. Mar Ecol Prog Ser, 338:307-310, 2007.

D. A. Mann, J. V. Locascio, F. C. Coleman, and C. C. Koenig. Goliath grouper Epinephelus itajara sound production and movement patterns on aggregation sites. Endanger Species Res, 7:229-236, 2009.

K. P. Maruska, K. S. Boyle, L. R. Dewan, and T. C. Tricas. Sound production and spectral hearing sensitivity in the Hawaiian sergeant damselfish, Abudefduf abdominalis. $J$ Exp Biol, 210:3990-4004, 2007.

M. I. McCormick. Comparison of field methods for measuring surface topography and their associations with a tropical reef fish assemblage. Mar Ecol Prog Ser, 112:87-96, 1994.

V. Parravicini, M. Kulbicki, D. R. Bellwood, A. M. Friedlander, J. E. Arias-Gonzalez, P. Chabanet, S. R. Floeter, R. Myers, L. Vigliola, S. D'Agata, and D. Mouillot. Global patterns and predictors of tropical reef fish species richness. Ecography, 36(12):1254-1262, 2013.

R. D. McCauley and D. H. Cato. Patterns of fish calling in a nearshore environment in the Great Barrier Reef. Philos Trans R Soc Lond B Biol Sci, 355(1401):1289-1293, 2000.

R. D. McCauley, J. Fewtrell, and A. N. Popper. High intensity anthropogenic sound damages fish ears. J Acoust Soc Am, 113(1):638-642, 2003.

M. A. McDonald, J. A. Hildebrand, and S. M. Wiggins. Increases in deep ocean ambient noise in the northeast pacific west of San Nicolas Island, California. J Acoust Soc Am, 120(2):711-718, 2006.

M. F. McKenna, D. Ross, S. M. Wiggins, and J. A. Hildebrand. Underwater radiated noise from modern commercial ships. J Acoust Soc Am, 131(1):92-103, 2012.

M. F. McKenna, S. M. Wiggins, and J. A. Hildebrand. Relationship between container ship underwater noise levels and ship design, operational and oceanographic conditions. Sci Rep, 3, 2013.

J. N. McWilliam and A. D. Hawkins. A comparison of inshore marine soundscapes. $J$ Exp Mar Biol Ecol, 446:166-176, 2013. 
H. Medwin and J. E. Blue. Sounds in the sea: From ocean acoustics to acoustical oceanography. Cambridge University Press, Cambridge, UK, 2005.

N. D. Merchant, K. M. Fristrup, M. P. Johnson, P. L. Tyack, M. J. Witt, P. Blondel, S. E. Parks, and D. Hodgson. Measuring acoustic habitats. Methods Ecol Evol, 6(3):257-265, 2015 .

V. Messmer, G. P. Jones, P. L. Munday, S. J. Holbrook, R. J. Schmitt, and A. Brooks. Habitat biodiversity as a determinant of fish community structure on coral reefs. Ecology, 92(12):2285-2298, 2011.

J. C. Montgomery, A. Jeffs, S. D. Simpson, M. Meekan, and C. Tindle. Sound as an orientation cue for the pelagic larvae of reef fishes and decapod crustaceans. Adv Mar Biol, 51:143-196, 2006.

T. A. Mooney, R. T. Hanlon, J. Christensen-Dalsgaard, P. T. Madsen, D. R. Ketten, and P. E. Nachtigall. Sound detection by the longfin squid (Loligo pealeii) studied with auditory evoked potentials: sensitivity to low-frequency particle motion and not pressure. $J$ Exp Biol, 213:3748-59, 2010.

H. Mouritsen, J. Atema, M. J. Kingsford, and G. Gerlach. Sun compass orientation helps coral reef fish larvae return to their natal reef. PLoS One, 8(6):e66039, 2013.

A. A. Myrberg. Sound production by a coral reef fish (Pomacentrus partitus): Evidence for a vocal territorial "keep-out" signal. Bull Mar Sci, 60(3):1017-1025, 1997.

P. E. Nachtigall, A. Y. Supin, J. Pawloski, and W. W. L. Au. Temporary threshold shifts after noise exposure in the bottlenose dolphin (Tursiops truncatus) measured using evoked auditory potentials. Mar Mam Sci, 20(4):673-687, 2004.

S. L. Nedelec, A. N. Radford, S. D. Simpson, B. Nedelec, D. Lecchini, and S. C. Mills. Anthropogenic noise playback impairs embryonic development and increases mortality in a marine invertebrate. Sci Rep, 4:5891, 2014.

S. L. Nedelec, S. D. Simpson, M. Holderied, A. N. Radford, G. Lecellier, C. Radford, and D. Lecchini. Soundscapes and living communities in coral reefs: temporal and spatial variation. Mar Ecol Prog Ser, 524:125-135, 2015.

S. L. Nedelec, J. Campbell, A. N. Radford, S. D. Simpson, N. D. Merchant, and D. Fisher. Particle motion: the missing link in underwater acoustic ecology. Methods Ecol Evol, 7 (7):836-842, 2016.

D. P. Nowacek, L. H. Thorne, D. W. Johnston, and P. L. Tyack. Responses of cetaceans to anthropogenic noise. Mammal Rev, 37(2):81-115, 2007.

A. Packard, H. E. Karlsen, and O. Sand. Low frequency hearing in cephalopods. J Comp Physiol A, 166:501-505, 1990.

J. M. Pandolfi, R. H. Bradbury, E. Sala, T. P. Hughes, K. A. Bjorndal, R. G. Cooke, D. McArdle, L. McClenachan, M. J. H. Newman, G. Paredes, R. R. Warner, and J. B. C. Jackson. Global trajectories of the long-term decline of coral reef ecosystems. Science, 301(5635):955-958, 2003. 
C. B. Paris and R. K. Cowen. Direct evidence of a biophysical retention mechanism for coral reef fish larvae. Limnol Oceanogr, 49(6):1964-1979, 2004.

S. E. Parks, M. Johnson, D. Nowacek, and P. L. Tyack. Individual right whales call louder in increased environmental noise. Biol. Lett., 7(1):33-5, 2011.

S. E. Parks, J. L. Miksis-Olds, and S. L. Denes. Assessing marine ecosystem acoustic diversity across ocean basins. Ecol Inform, 21:81-88, 2014.

E. Parmentier, J. P. Lagardere, J. B. Braquegnier, P. Vandewalle, and M. L. Fine. Sound production mechanism in carapid fish: first example with a slow sonic muscle. $J$ Exp Biol, 209:2952-60, 2006.

E. Parmentier, L. Kever, M. Casadevall, and D. Lecchini. Diversity and complexity in the acoustic behaviour of Dacyllus flavicaudus (Pomacentridae). Mar Biol, 157:2317-2327, 2010 .

E. Parmentier, L. Berten, P. Rigo, F. Aubrun, S. Nedelec, S. D. Simpson, and D. Lecchini. The influence of various reef sounds on coral-fish larvae behaviour. J Fish Biol, 86(5): 1507-1518, 2015.

J. J. B. Piercy, E. A. Codling, A. J. Hill, D. J. Smith, and S. D. Simpson. Habitat quality affects sound production and likely distance of detection on coral reefs. Mar Ecol Prog Ser, 516:35-47, 2014.

N. Pieretti, A. Farina, and D. Morri. A new methodology to infer the singing activity of an avian community: The Acoustic Complexity Index (ACI). Ecol Indic, 11(3):868-873, 2011.

B. C. Pijanowski, L. J. Villanueva-Rivera, S. L. Dumyahn, A. Farina, B. L. Krause, B. M. Napoletano, S. H. Gage, and N. Pieretti. Soundscape ecology: The science of sound in the landscape. BioScience, 61(3):203-216, 2011.

M. K. Pine, A. G. Jeffs, and C. A. Radford. Turbine sound may influence the metamorphosis behaviour of estuarine crab megalopae. PLoS One, 7(12):e51790, 2012.

L. Plaisance, M. J. Caley, R. E. Brainard, and N. Knowlton. The diversity of coral reefs: What are we missing. PLoS ONE, 6:e25026, 2011.

A. N. Popper and R. R. Fay. Rethinking sound detection by fishes. Hear Res, 273(1-2): 25-36, 2011.

A. N. Popper and M. C. Hastings. The effects of anthropogenic sources of sound on fishes. J Fish Biol, 75(3):455-489, 2009.

A. N. Popper, M. E. Smith, P. A. Cott, B. W. Hanna, A. O. MacGillivray, M. E. Austin, and D. A. Mann. Effects of exposure to seismic airgun use on hearing of three fish species. $J$ Acoust Soc Am, 117(6):3958-3971, 2005.

A.N. Popper and W. N Tavolga. Structure and function of the ear in the marine catfish, Arius felis. J Comp Physiol, 144:27-34, 1981. 
Port of Vancouver. Underwater listening station launched to better understand impact of ship noise on at-risk whales, 2015. URL https://www.portvancouver.com/newsand-media/news/underwater-listening-station-launched-to-better-understandimpact-of-ship-noise-on-at-risk-whales/.

C. A. Radford, A. G. Jeffs, C. T. Tindle, and J. C. Montgomery. Temporal patterns in ambient noise of biological origin from a shallow water temperate reef. Oecologia, 156(4): 921-929, 2008.

C. A. Radford, J. A. Stanley, C. T. Tindle, J. C. Montgomery, and A. G. Jeffs. Localised coastal habitats have distinct underwater sound signatures. Mar Ecol Prog Ser, 401:21-29, 2010 .

C. A. Radford, J. A. Stanley, S. D. Simpson, and A. G. Jeffs. Juvenile coral reef fish use sound to locate habitats. Coral Reefs, 30(2):295-305, 2011a.

C. A. Radford, C. T. Tindle, J. C. Montgomery, and A. G. Jeffs. Modelling a reef as an extended sound source increases the predicted range at which reef noise may be heard by fish larvae. Mar Ecol Prog Ser, 438:167-174, 2011b.

C. A. Radford, J. C. Montgomery, P. Caiger, and D. M. Higgs. Pressure and particle motion detection thresholds in fish: a re-examination of salient auditory cues in teleosts. $J$ Exp Biol, 215:3429-3435, 2012.

C. A. Radford, J. A. Stanley, and A. G. Jeffs. Adjacent coral reef habitats produce different underwater sound signatures. Mar Ecol Prog Ser, 505:19-28, 2014.

C. A. Radford, S. Ghazali, A. G. Jeffs, and J. C. Montgomery. Vocalisations of the bigeye, Pempheris adspersa: Characteristics, source level, and active space. J Exp Biol, 218: 940-948, 2015.

W. Rayment, S. Dawson, and L. Slooten. Use of T-PODs for acoustic monitoring of Cephalorhynchus dolphins: a case study with Hector's dolphins in a marine protected area. Endanger Species Res, 10:333-339, 2009.

M. L. Reaka-Kudla. The global biodiversity of coral reefs: a comparison with rain forests. In M. L. Reaka-Kudla, D. E. Wilson, and E. O. Wilson, editors, Biodiversity II: Understanding and Protecting Our Biological Resources, number 7, pages 83-108. Joseph Henry Press, Washington, DC, 1997.

W. J. Richardson. Marine Mammals and Noise. Academic Press, San Diego, CA, 1995.

R. H. Richmond and C. L. Hunter. Reproduction and recruitment of corals: comparisons among the Caribbean, the Tropical Pacific, and the Red Sea. Mar Ecol Prog Ser, 60: 185-203, 1990.

L. Roberts, S. Cheesman, T. Breithaupt, and M. Elliott. Sensitivity of the mussel Mytilus edulis to substrate-borne vibration in relation to anthropogenically generated noise. Mar Ecol Prog Ser, 538:185-195, 2015.

P. H. Rogers and M. Cox. Underwater Sound as a Biological Stimulus, book section 5, pages 131-149. Springer-Verlag, New York, 1988. 
R. M. Rolland, S. E. Parks, K. E. Hunt, M. Castellote, P. J. Corkeron, D. P. Nowacek, S. K. Wasser, and S. D. Kraus. Evidence that ship noise increases stress in right whales. Proc Biol Sci, 279(1737):2363-2368, 2012.

A. Sirović, J. A. Hildebrand, and M. A. McDonald. Ocean ambient sound south of Bermuda and Panama Canal traffic. J Acoust Soc Am, 139(5):2417-2423, 2016.

L. Ruppe, G. Clement, A. Herrel, L. Ballesta, T. Decamps, L. Kever, and E. Parmentier. Environmental constraints drive the partitioning of the soundscape in fishes. Proc Natl Acad Sci U S A, 2015.

J. E. Samson, T. A. Mooney, S. W. Gussekloo, and R. T. Hanlon. Graded behavioral responses and habituation to sound in the common cuttlefish Sepia officinalis. $J$ Exp Biol, 217:4347-4355, 2014.

G. Sara, J. M. Dean, D. D'Amato, G. Buscaino, A. Oliveri, S. Genovese, S. Ferro, G. Buffa, M. Lo Martire, and S. Mazzola. Effect of boat noise on the behaviour of bluefin tuna Thunnus thynnus in the Mediterranean Sea. Mar Ecol Prog Ser, 331:243-253, 2007.

S. D. Simpson, M. G. Meekan, R. D. McCauley, and A. Jeffs. Attraction of settlement-stage coral reef fishes to reef noise. Mar Ecol Prog Ser, 276:263-268, 2004.

S. D. Simpson, M. G. Meekan, J. Montgomery, R. D. McCauley, and A. Jeffs. Homeward sound. Science, 308:221, 2005.

S. D. Simpson, M. G. Meekan, A. Jeffs, J. Montgomery, and R. D. McCauley. Settlementstage coral reef fish prefer the higher-frequency invertebrate-generated audible component of reef noise. Anim Behav, 75(6):1861-1868, 2008.

S. D. Simpson, J. Purser, and A. N. Radford. Anthropogenic noise compromises antipredator behaviour in european eels. Glob Chang Biol, 21(2):586-593, 2015.

S. D. Simpson, A. N. Radford, S. L. Nedelec, M. C. Ferrari, D. P. Chivers, M. I. McCormick, and M. G. Meekan. Anthropogenic noise increases fish mortality by predation. Nat Commun, 7:10544, 2016.

H. Slabbekoorn, N. Bouton, I. van Opzeeland, A. Coers, C. ten Cate, and A. N. Popper. A noisy spring: the impact of globally rising underwater sound levels on fish. Trends Ecol Evol, 25(7):419-27, 2010.

M. E. Smith, A. S. Kane, and A. N. Popper. Noise-induced stress response and hearing loss in goldfish. J Exp Biol, 207:427-435, 2004.

E. Staaterman, A. N. Rice, D. A. Mann, and C. B. Paris. Soundscapes from a tropical eastern Pacific reef and a Caribbean sea reef. Coral Reefs, 32(2):553-557, 2013.

E. Staaterman, C. B. Paris, H. A. DeFerrari, D. A. Mann, A. N. Rice, and E. K. D'Alessandro. Celestial patterns in marine soundscapes. Mar Ecol Prog Ser, 508:17$32,2014$.

J. A. Stanley, C. A. Radford, and A. G. Jeffs. Induction of settlement in crab megalopae by ambient underwater reef sound. Behav Ecol, 21(1):113-120, 2009. 
J. A. Stanley, C. A. Radford, and A. G. Jeffs. Behavioural response thresholds in New Zealand crab megalopae to ambient underwater sound. PLoS One, 6(12):e28572, 2011.

I. C. Stobutzki and D. R. Bellwood. Sustained swimming abilities of the late pelagic stages of coral reef fishes. Mar Ecol Prog Ser, 149(1-3):35-41, 1997.

J. Sueur, S. Pavoine, O Hamerlynck, and S. Duvail. Rapid acoustic survey for biodiversity appraisal. PLoS ONE, 3(12):e4065, 2008.

A. M. Szmant. Nutrient enrichment on coral reefs: Is it a major cause of coral reef decline? Estuaries, 25(4):743-766, 2002.

D. P. Tittensor, C. Mora, W. Jetz, H. K. Lotze, D. Ricard, E. V. Berghe, and B. Worm. Global patterns and predictors of marine biodiversity across taxa. Nature, 466(7310): 1098-1101, 2010.

N. Tolimieri, A. Jeffs, and J. C. Montgomery. Ambient sound as a cue for navigation by the pelagic larvae of reef fishes. Mar Ecol Prog Ser, 207:219-224, 2000.

N. Tolimieri, O. Haine, A. Jeffs, R. McCauley, and J. Montgomery. Directional orientation of pomacentrid larvae to ambient reef sound. Coral Reefs, 23:184-191, 2004.

T. C. Tricas and K. S. Boyle. Acoustic behaviors in hawaiian coral reef fish communities. Mar Ecol Prog Ser, 511:1-16, 2014.

P. L. Tyack. Acoustic communication under the sea. In S. L. Hopp, M. J. Owren, and C. S. Evans, editors, Animal Acoustic Communication: Sound Analysis and Research Methods, book section 6, page 421. Springer, Berlin, Germany, 1998.

UNCTAD. Review of maritime transport 2015. Report, UCNTAD, 2015.

UNCTAD Statistics. Ucntad statistics dataset, 2016. URL http://unctadstat.unctad. org/wds/.

R. J. Urick. Principles of Underwater Sound. McGraw-Hill, New York, NY, 1983.

R. J. Urick. Ambient noise in the sea. Report, Naval Sea Systems Command, 1984.

M. J. A. Vermeij, K. L. Marhaver, C. M. Huijbers, I. Nagelkerken, and S. D. Simpson. Coral larvae move toward reef sounds. PloS ONE, 5(5):e10660, 2010.

M. Versluis. How snapping shrimp snap: Through cavitating bubbles. Science, 289(5487): 2114-2117, 2000.

M. A. Wale, S. D. Simpson, and A. N. Radford. Noise negatively affects foraging and antipredator behaviour in shore crabs. Animal Behaviour, 86(1):111-118, 2013.

W. J. Walsh. Patterns of recruitment and spawning in Hawaiian reef fishes. Environ Biol Fishes, 18(4):257-276, 1987.

P. D. Welch. The use of fast fourier transform for the estimation of power spectra: A method based on time averaging over short, modified periodograms. IEEE Trans. Acoust., AU-15 (2):70-73, 1967. 
I. D. Williams, J. K. Baum, A. Heenan, K. M. Hanson, M. O. Nadon, and R. E. Brainard. Human, oceanographic and habitat drivers of central and western pacific coral reef fish assemblages. PLoS One, 10(4):e0120516, 2015.

S. K. Wilson, N. A. J. Graham, M. S. Pratchett, G. P. Jones, and N. V. C. Polunin. Multiple disturbances and the global degradation of coral reefs: are reef fishes at risk or resilient? Glob Change Biol, 12(11):2220-2234, 2006.

WMO. Climate, carbon and coral reefs. Report WMO-No. 1063, World Meterological Organization, 2010.

L. E. Wysocki and F. Ladich. Hearing in fishes under noise conditions. J Assoc Res Otolaryngol, 6(1):28-36, 2005.

L. E. Wysocki, J. P. Dittami, and F. Ladich. Ship noise and cortisol secretion in European freshwater fishes. Biol Cons, 128(4):501-508, 2006.

D. G. Zeddies, R. R. Fay, M. D. Gray, P. W. Alderks, A. Acob, and J. A. Sisneros. Local acoustic particle motion guides sound-source localization behavior in the plainfin midshipman fish, Porichthys notatus. J Exp Biol, 215:152-60, 2012.

W. M. X. Zimmer. Passive Acoustic Monitoring of Cetaceans. University of Cambridge Press, Cambridge, UK, 2011. 